\title{
Cardy Algebras and Sewing Constraints, I
}

\author{
Liang Kong ${ }^{1,2}$, Ingo Runkel ${ }^{3}$ \\ ${ }^{1}$ Max-Planck-Institut für Mathematik, Vivatsgasse 7, 53111 Bonn, Germany. \\ E-mail: kong@mpim-bonn.mpg.de \\ 2 Hausdorff Research Institute for Mathematics, Poppelsdorfer Allee 45, 53115 Bonn, Germany \\ 3 Department of Mathematics, King's College London, Strand, London WC2R 2LS, United Kingdom. \\ E-mail: ingo.runkel@kcl.ac.uk
}

\begin{abstract}
This is part one of a two-part work that relates two different approaches to two-dimensional open-closed rational conformal field theory. In part one we review the definition of a Cardy algebra, which captures the necessary consistency conditions of the theory at genus 0 and 1 . We investigate the properties of these algebras and prove uniqueness and existence theorems. One implication is that under certain natural assumptions, every rational closed CFT is extendable to an open-closed CFT. The relation of Cardy algebras to the solutions of the sewing constraints is the topic of part two.
\end{abstract}

\section{Contents}

1. Introduction and Summary . . . . . . . . . . . . . . . . 871

2. Preliminaries on Tensor Categories . . . . . . . . . . . . . . . 875

2.1 Tensor categories and (co)lax tensor functors . . . . . . . . . . . . 875

2.2 Algebras in tensor categories . . . . . . . . . . . . . . . . . . 878

2.3 Modular tensor categories . . . . . . . . . . . . . . . . . . . 884

2.4 The functors $T$ and $R \ldots \ldots \ldots$. . . . . . . . . . . . . 885

3. Cardy Algebras . . . . . . . . . . . . . . . . . . . . 891

3.1 Modular invariance . . . . . . . . . . . . . . . . . . . . 891

3.2 Two definitions . . . . . . . . . . . . . . . . . . . 895

3.3 Uniqueness and existence theorems . . . . . . . . . . . . . . . . 899

A. Appendix . . . . . . . . . . . . . . . . . 905

A.1 Proof of Lemma 2.7 . . . . . . . . . . . . . . . . . . . . . 905

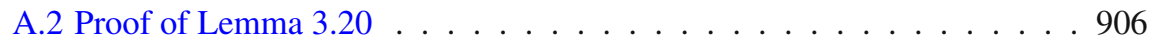

\section{Introduction and Summary}

This is part I of a two-part work which relates two different approaches to two-dimensional open-closed rational conformal field theory (CFT). 
The first approach uses a three-dimensional topological field theory to express correlators of the open-closed CFT [Fe,FRS, Fj]. Here one starts from a modular tensor category, which defines a three-dimensional topological field theory [RT,T], and from a special symmetric Frobenius algebra in this modular tensor category. To each openclosed world sheet $X$ one assigns a 3-bordism $M_{X}$ with embedded ribbon graph constructed from this Frobenius algebra. To the boundary of $M_{X}$ the topological field theory assigns a vector space $B \ell(X)$ and to $M_{X}$ itself a vector $C_{X} \in B \ell(X)$. One proves that this collection of vectors $C_{X}$ provides a so-called solution to the sewing constraints [Fj]. If the modular tensor category is the category of representations of a suitable vertex operator algebra, the spaces $B \ell(X)$ are spaces of conformal blocks, and the $C_{X}$ are the correlators of an open-closed CFT. In this approach one thus makes an ansatz for the correlators on all world sheets simultaneously and then proves that they obey the necessary consistency conditions. The relation to CFT rests on convergence and factorisation properties of higher genus conformal blocks, and the precise list of conditions the vertex operator algebra has to fulfill for these properties to hold is not known. However, from a physical perspective one expects that interesting classes of models [W,FK] will have all the necessary properties.

The second approach uses the theory of vertex operator algebras to construct directly the correlators of the genus 0 and genus 1 open-closed CFT [HK1,HK2,K3]. More precisely, in this approach one uses a notion of CFT defined in [K3, Sect. 1] (and called partial $\mathrm{CFT}^{1}$ there), where one glues Riemann surfaces around punctures with local coordinates as in [V,H1] instead of gluing around parametrised circles as in [Se]. This approach is based on the precise relation between genus- 0 CFT and vertex operator algebras [H1], and on the fact that the category of modules over a rational vertex operator algebra is a modular tensor category [HL,H2]. Let us call a vertex operator algebra rational if it satisfies the conditions in [H2, Sect. 1]. If one analyses the consistency conditions of a genus-0,1 open-closed CFT, one arrives at a structure called Cardy $\mathcal{C}_{V} \mid \mathcal{C}_{V \otimes V}$ - algebra in [K3]. It is formulated in purely categorical terms in the categories $\mathcal{C}_{V}$ and $\mathcal{C}_{V \otimes V}$ of modules over the rational vertex operator algebras $V$ and $V \otimes V$, respectively. Cardy algebras (Definition 3.7) are the central objects in part I of this work, and we will describe their relation to CFT in slightly more detail below. The data in a Cardy algebra amounts to an open-closed CFT on a generating set of world sheets, from which the entire CFT can be obtained by repeated gluing. The conditions on this data are necessary for this procedure to give a consistent genus- 0,1 open-closed CFT.

The two approaches just outlined start at opposite ends of the same problem. In both cases the difficulty to obtain a complete answer lies in the lack of control over the properties of higher genus conformal blocks. Nonetheless, both approaches give rise to notions formulated in entirely categorical terms, and we can compare the structures one finds. In part II we will come to the satisfying conclusion that giving a solution to the sewing constraints is essentially equivalent, in a sense made precise in part II, to giving a Cardy algebra.

To motivate the notion of a Cardy algebra and our interest in it, we would like to outline how it emerges when formulating closed CFT and open-closed CFT at genus-0,1 in the language of vertex operator algebras. The next one and a half pages, together with a few

1 The qualifier 'partial' refers to the fact that the gluing of punctures is only defined if the coordinates $\zeta_{1}$, $\zeta_{2}$ around two punctures can be analytically extended to a large enough region containing no other punctures, so that the identification $\zeta_{1} \sim 1 / \zeta_{2}$ is well-defined. That is, if $\zeta_{1}$ can be extended to a disc of radius $r$, then $\zeta_{2}$ must be defined on a disc of radius greater than $1 / r$. Both discs must not contain further punctures. 
remarks in the main text, are the only places where we make reference to vertex operator algebras. The reader who is not familiar with this structure is invited to skip ahead.

All types of field algebras occurring below are called self-dual if they are endowed with non-degenerate invariant bilinear forms.

A genus-0 closed CFT is equivalent to an algebra over a partial dioperad consisting of spheres with arbitrary in-coming and out-going punctures. The dioperad structure allows to compose one in-going and one out-going puncture of distinct spheres, so that the result is again a sphere. Such an algebra with additional natural properties is canonically equivalent to a so-called self-dual conformal full field algebra [HK2,K1]. A conformal full field algebra contains chiral and anti-chiral parts, the easiest nontrivial example is given by $V \otimes V$, where $V$ is a vertex operator algebra. A conformal full field algebra containing $V \otimes V$ as a subalgebra is called a conformal full field algebra over $V \otimes V$. When $V$ is rational, the category of self-dual conformal full field algebras over $V \otimes V$ is isomorphic to the category of commutative symmetric Frobenius algebras in $\mathcal{C}_{V \otimes V}$ [K1, Thm. 4.15].

Similarly, a genus-0 open CFT is an algebra over a partial dioperad consisting of disks with an arbitrary number of in-coming and out-going boundary punctures. Such an algebra with additional natural properties is canonically equivalent to a self-dual open-string vertex operator algebra as defined in [HK1]. A vertex operator algebra $V$ is naturally an open-string vertex operator algebra. An open-string vertex operator algebra containing $V$ as a subalgebra in its meromorphic centre is called an open-string vertex operator algebra over $V$. When $V$ is rational, the category of self-dual open-string vertex operator algebras over $V$ is isomorphic to the category of symmetric Frobenius algebras in $\mathcal{C}_{V}$, see [HK1, Thm. 4.3] and [K3, Thm. 6.10].

Finally, a genus- 0 open-closed CFT is an algebra over the Swiss-cheese partial dioperad, which consists of disks with both interior punctures and boundary punctures, and is equipped with an action of the partial spheres dioperad. Such an algebra can be constructed from a so-called self-dual open-closed field algebra [K2]. It consists of a self-dual conformal full field algebra $A_{\mathrm{cl}}$, a self-dual open-string vertex operator algebra $A_{\text {op }}$, and interactions between $A_{\mathrm{cl}}$ and $A_{\mathrm{op}}$ satisfying certain compatibility conditions. Namely, if $A_{\mathrm{cl}}$ is defined over $V \otimes V$ and $A_{\mathrm{op}}$ over $V$, one requires that the boundary condition on a disc is $V$-invariant in the sense that both the chiral copy $V \otimes \mathbf{1}$ and the anti-chiral copy $\mathbf{1} \otimes V$ of $V$ in $A_{\mathrm{cl}}$ give the copy of $V$ in $A_{\mathrm{op}}$ in the limit of the insertion point approaching a point on the boundary of the disc [K2, Def. 1.25]. An open-closed field algebra with $V$-invariant boundary condition is called an open-closed field algebra over $V$. When $V$ is rational, the category of self-dual open-closed field algebras over $V$ is isomorphic to the category of triples $\left(A_{\mathrm{op}} \mid A_{\mathrm{cl}}, \tilde{\iota}_{\mathrm{cl}-\mathrm{op}}\right)$, where $A_{\mathrm{cl}}$ is a commutative symmetric Frobenius $\mathcal{C}_{V \otimes V}$-algebra, $A_{\text {op }}$ a symmetric Frobenius $\mathcal{C}_{V}$-algebra, and $\tilde{\iota}_{\text {cl-op }}$ an algebra homomorphism $T\left(A_{\mathrm{cl}}\right) \rightarrow A_{\mathrm{op}}$ satisfying a centre condition (given in (3.20) below), see [K2, Thm.3.14] and [K3, Sect.6.2]. Here $T: \mathcal{C}_{V \otimes V} \rightarrow \mathcal{C}_{V}$ is the Huang-Lepowsky tensor product functor [HL].

The genus- 1 theory does not provide new data as it is determined by taking traces of genus- 0 correlators, but it does provide two additional consistency conditions: the modular invariance condition for one-point correlators on the torus [So], and the Cardy condition for boundary-two-point correlators on the annulus [C2,Lw]. Their categorical formulations have been worked out in [HK3, K3]. Adding them to the axioms of a self-dual open-closed field algebra over $V$ finally results in the notion of a Cardy $\mathcal{C}_{V} \mid \mathcal{C}_{V \otimes V}$-algebra. One can prove that the category of self-dual open-closed field algebras over a rational vertex operator algebra $V$ satisfying the two genus-1 consistency conditions is isomorphic to the category of Cardy $\mathcal{C}_{V} \mid \mathcal{C}_{V \otimes V}$-algebras [K3, Thm. 6.15]. 
If $V$ is rational, then so is $V \otimes V$ [DMZ,HK2]. Thus both $\mathcal{C}_{V}$ and $\mathcal{C}_{V \otimes V}$ are modular tensor categories. In fact, $\mathcal{C}_{V \otimes V} \cong \mathcal{C}_{V} \otimes\left(\mathcal{C}_{V}\right)_{-}$(see [FHL, Thm. 4.7.4] and [DMZ, Thm 2.7]), where the minus sign relates to the particular braiding used for $\mathcal{C}_{V \otimes V}$. Namely, for a given modular tensor category $\mathcal{D}$, we denote by $\mathcal{D}_{-}$the modular tensor category obtained from $\mathcal{D}$ by inverting braiding and twist. We will also sometimes write $\mathcal{D}_{+}$for $\mathcal{D}$. The product $\otimes$ amounts to taking direct sums of pairs of objects and tensor products of morphisms spaces. The definition of a Cardy algebra can be stated in a way that no longer makes reference to the vertex operator algebra $V$, and therefore makes sense in an arbitrary modular tensor category $\mathcal{C}$. Abbreviating $\mathcal{C}_{ \pm}^{2} \equiv \mathcal{C}_{+} \otimes \mathcal{C}_{-}$, this leads to the definition of a Cardy $\mathcal{C} \mid \mathcal{C}_{ \pm}^{2}$-algebra.

The relation to genus- 0,1 open-closed CFT outlined above is the main motivation for our interest in Cardy $\mathcal{C} \mid \mathcal{C}_{ \pm}^{2}$-algebras. In part I of this work we investigate how much one can learn about Cardy algebras in the categorical setting, and without the assumption that the modular tensor category $\mathcal{C}$ is given by $\mathcal{C}_{V}$ for some $V$. We briefly summarise our approach and results below.

In Sect. 2.1-2.3, we recall some basic notions we will need, such as (co)lax tensor functors, Frobenius functors, and modular tensor categories. In Sect. 2.4, we study the functor $T: \mathcal{C}_{ \pm}^{2} \rightarrow \mathcal{C}$, which is defined by the tensor product on $\mathcal{C}$ via $T\left(\oplus_{i} A_{i} \times B_{i}\right)=$ $\oplus_{i} A_{i} \otimes B_{i}$ for $A_{i}, B_{i} \in \mathcal{C}$. Using the braiding of $\mathcal{C}$ one can turn $T$ into a tensor functor. A tensor functor is automatically also a Frobenius functor, and so takes a Frobenius algebra $A$ in its domain category to a Frobenius algebra $F(A)$ in its target category.

An important object in this work is the functor $R: \mathcal{C} \rightarrow \mathcal{C}_{ \pm}^{2}$, also defined in Sect. 2.4. We show that $R$ is left and right adjoint to $T$. As a consequence, $R$ is automatically a lax and colax tensor functor, but it is in general not a tensor functor. However, we will show that it is still a Frobenius functor, and so takes Frobenius algebras in $\mathcal{C}$ to Frobenius algebras in $\mathcal{C}_{ \pm}^{2}$. In fact, it also preserves the properties simple, special and symmetric of a Frobenius algebra. In the case $\mathcal{C}=\mathcal{C}_{V}$ the functor $R: \mathcal{C}_{V} \rightarrow \mathcal{C}_{V \otimes V}$ was first constructed in [Li1,Li2] using techniques from vertex operator algebras. This motivated the present construction and notation. The functor $R$ was also considered in a slightly different context in [ENO2].

The above results imply that $R$ and $T$ form an ambidextrous adjunction, and we will use this adjunction to transport algebraic structures between $\mathcal{C}$ and $\mathcal{C}_{ \pm}^{2}$. For example, the algebra homomorphism $\tilde{\iota}_{\mathrm{cl}-\mathrm{op}}: T\left(A_{\mathrm{cl}}\right) \rightarrow A_{\text {op }}$ in $\mathcal{C}$ is transported to an algebra homomorphism $\iota_{\mathrm{cl} \text {-op }}: A_{\mathrm{cl}} \rightarrow R\left(A_{\mathrm{op}}\right)$ in $\mathcal{C}_{ \pm}^{2}$. This gives rise to an alternative definition of a Cardy $\mathcal{C} \mid \mathcal{C}_{ \pm}^{2}$-algebra as a triple $\left(A_{\text {op }} \mid A_{\mathrm{cl}}, \iota_{\mathrm{cl}-\text { op }}\right)$.

To prepare the definition of a Cardy algebra, in Sect. 3.1 we discuss the so-called modular invariance condition for algebras in $\mathcal{C}_{ \pm}^{2}$ (Definition 3.1 below). We show that when $A_{\mathrm{cl}}$ is simple, the modular invariance condition can be replaced by an easier condition on the quantum dimension of $A_{\mathrm{cl}}$ (namely, the dimension of $A_{\mathrm{cl}}$ has to be that of the modular tensor category $\mathcal{C}$ ), see Theorem 3.4.

In Sect. 3.2 we give the two definitions of a Cardy algebra and prove their equivalence. Section 3.3 contains our main results. We first show that for each special symmetric Frobenius algebra $A$ in $\mathcal{C}$ (see Sect. 2.2 for the definition of special) one obtains a Cardy algebra $(A \mid Z(A), e)$, where $Z(A)$ is the full centre of $A$ (Theorem 3.18). The full centre [Fj, Def.4.9] is a subobject of $R(A)$ and $e: Z(A) \rightarrow R(A)$ is the canonical embedding. Next we prove a uniqueness theorem (Theorem 3.21), which states that if ( $\left.A_{\mathrm{op}} \mid A_{\mathrm{cl}}, \iota_{\mathrm{cl}-\mathrm{op}}\right)$ is a Cardy algebra such that $\operatorname{dim} A_{\mathrm{op}} \neq 0$ and $A_{\mathrm{cl}}$ is simple, then $A_{\mathrm{op}}$ is special and $\left(A_{\mathrm{op}} \mid A_{\mathrm{cl}}, \iota_{\mathrm{cl}-\mathrm{op}}\right)$ is isomorphic to $\left(A_{\mathrm{op}} \mid Z\left(A_{\mathrm{op}}\right), e\right)$. When combined with part II of this work, this result amounts to [Fj, Thm.4.26] and provides an alternative 
(and shorter) proof. Finally we show that for every simple modular invariant commutative symmetric Frobenius algebra $A_{\mathrm{cl}}$ in $\mathcal{C}_{ \pm}^{2}$ there exists a simple special symmetric Frobenius algebra $A_{\mathrm{op}}$ and an algebra homomorphism $\iota_{\mathrm{cl}-\mathrm{op}}: A_{\mathrm{cl}} \rightarrow R\left(A_{\mathrm{op}}\right)$ such that $\left(A_{\mathrm{op}}, A_{\mathrm{cl}}, \iota_{\mathrm{cl}-\mathrm{op}}\right)$ is a Cardy algebra (Theorem 3.22). This theorem is closely related to a result announced in [Mü2] and provides an independent proof in the framework of Cardy algebras.

In physical terms these two theorems mean that a rational open-closed CFT with a unique closed vacuum state can be uniquely reconstructed from its correlators involving only discs with boundary punctures, and that every closed CFT with unique vacuum and left/right rational chiral algebra $V \otimes V$ occurs as part of such an open-closed CFT.

\section{Preliminaries on Tensor Categories}

In this section, we review some basic facts of tensor categories and fix our conventions and notations along the way.

2.1. Tensor categories and (co)lax tensor functors. In a tensor (or monoidal) category $\mathcal{C}$ with tensor product bifunctor $\otimes$ and unit object 1 , for $U, V, W \in \mathcal{C}$, we denote the associator $U \otimes(V \otimes W) \stackrel{\cong}{\rightarrow}(U \otimes V) \otimes W$ by $\alpha_{U, V, W}$, the left unit isomorphism $\mathbf{1} \otimes U \stackrel{\cong}{\rightarrow} U$ by $l_{U}$, and the right unit isomorphism $U \otimes \mathbf{1} \cong U$ by $r_{U}$. If $\mathcal{C}$ is braided, for $U, V \in \mathcal{C}$ we write the braiding isomorphism as $c_{U, V}: U \otimes V \rightarrow V \otimes U$.

Let $\mathcal{C}_{1}$ and $\mathcal{C}_{2}$ be two tensor categories with units $\mathbf{1}_{1}$ and $\mathbf{1}_{2}$ respectively. For simplicity, we will often write $\otimes, \alpha, l, r$ for the data of both $\mathcal{C}_{1}$ and $\mathcal{C}_{2}$. Lax and colax tensor functors are defined as follows, see e.g. [Y, Ch.I.3] or [Ln, Ch.I.1.2].

Definition 2.1. $A$ lax tensor functor $G: \mathcal{C}_{1} \rightarrow \mathcal{C}_{2}$ is a functor equipped with a morphism $\phi_{0}^{G}: \mathbf{1}_{2} \rightarrow G\left(\mathbf{1}_{1}\right)$ in $\mathcal{C}_{2}$ and a natural transformation $\phi_{2}^{G}: \otimes \circ(G \times G) \rightarrow G \circ \otimes$ such that the following three diagrams commute:
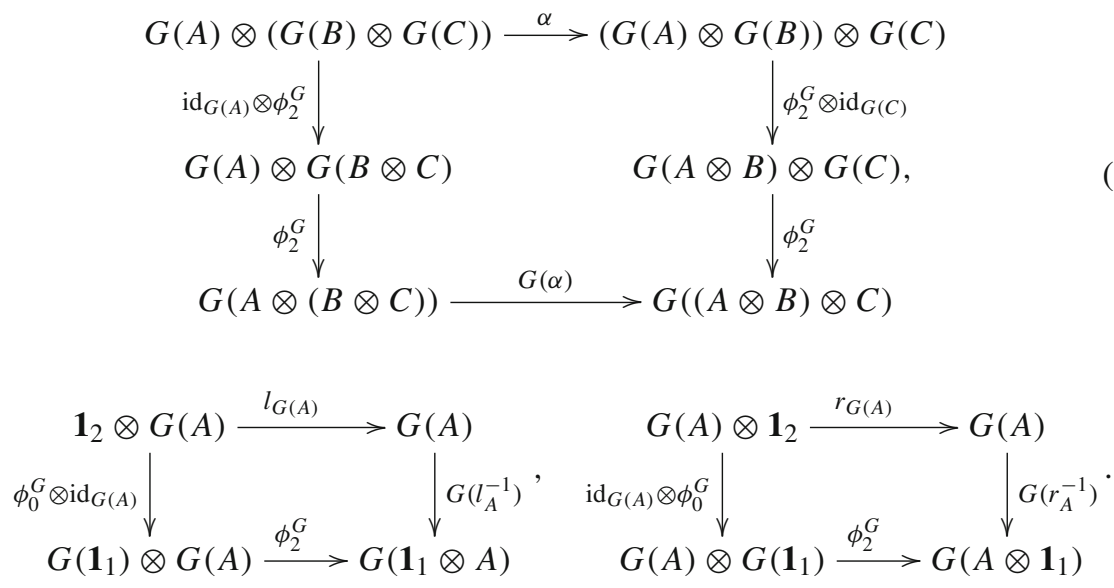

Definition 2.2. A colax tensor functor is a functor $F: \mathcal{C}_{1} \rightarrow \mathcal{C}_{2}$ equipped with a morphism $\psi_{0}^{F}: F\left(\mathbf{1}_{1}\right) \rightarrow \mathbf{1}_{2}$ in $\mathcal{C}_{2}$, and a natural transformation $\psi_{2}^{F}: F \circ \otimes \rightarrow \otimes \circ(F \times F)$ such that the following three diagrams commute: 

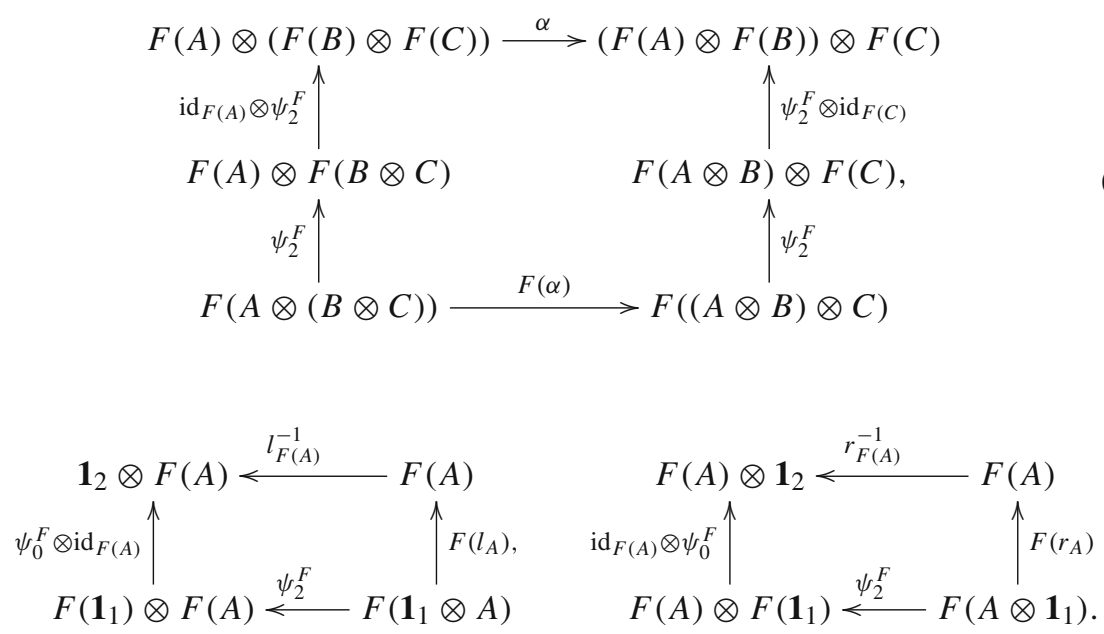

We denote a lax tensor functor by $\left(G, \phi_{2}^{G}, \phi_{0}^{G}\right)$ or just $G$, and a colax tensor functor by $\left(F, \psi_{2}^{F}, \psi_{0}^{F}\right)$ or $F$.

Definition 2.3. A tensor functor $T: \mathcal{C}_{1} \rightarrow \mathcal{C}_{2}$ is a lax tensor functor $\left(T, \phi_{2}^{T}, \phi_{0}^{T}\right)$ such that $\phi_{0}^{T}, \phi_{2}^{T}$ are both isomorphisms.

A tensor functor $\left(T, \phi_{2}^{T}, \phi_{0}^{T}\right)$ is automatically a colax tensor functor $\left(T, \psi_{2}^{T}, \psi_{0}^{T}\right)$ with $\psi_{0}^{T}=\left(\phi_{0}^{T}\right)^{-1}$ and $\psi_{2}^{T}=\left(\phi_{2}^{T}\right)^{-1}$.

In the next section we will discuss algebras in tensor categories. The defining properties (2.1) and (2.2) of a lax tensor functor are analogues of the associativity, the left-unit, and the right-unit properties of an algebra. Indeed, a lax tensor functor $G: \mathcal{C}_{1} \rightarrow \mathcal{C}_{2}$ maps a $\mathcal{C}_{1}$-algebra to a $\mathcal{C}_{2}$-algebra. Similarly, (2.3) and (2.4) are analogues of the coassociativity, the left-counit and the right-counit properties of a coalgebra, and a colax tensor functor $F: \mathcal{C}_{1} \rightarrow \mathcal{C}_{2}$ maps a $\mathcal{C}_{1}$-coalgebra to a $\mathcal{C}_{2}$-coalgebra. We will later make use of functors that take Frobenius algebras to Frobenius algebras. This requires a stronger condition than being lax and colax and leads to the notion of a 'functor with Frobenius structure' or 'Frobenius monoidal functor' [Sz,DP,P], which we will simply refer to as Frobenius functor.

Definition 2.4. $A$ Frobenius functor $F: \mathcal{C}_{1} \rightarrow \mathcal{C}_{2}$ is a tuple $F \equiv\left(F, \phi_{2}^{F}, \phi_{0}^{F}, \psi_{2}^{F}, \psi_{0}^{F}\right)$ such that $\left(F, \phi_{2}^{F}, \phi_{0}^{F}\right)$ is a lax tensor functor, $\left(F, \psi_{2}^{F}, \psi_{0}^{F}\right)$ is a colax tensor functor, and such that the following two diagrams commute:

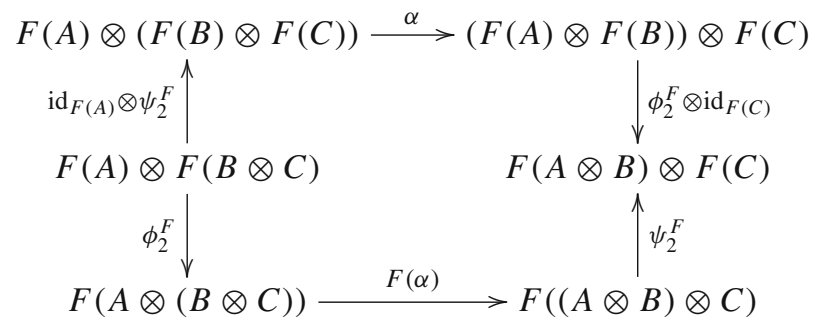




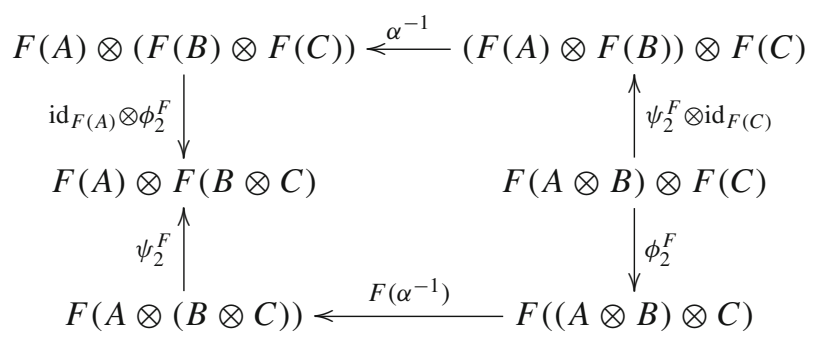

Proposition 2.5. If $\left(F, \phi_{2}^{F}, \phi_{0}^{F}\right)$ is a tensor functor, then $F$ is a Frobenius functor with $\psi_{0}^{F}=\left(\phi_{0}^{F}\right)^{-1}$ and $\psi_{2}^{F}=\left(\phi_{2}^{F}\right)^{-1}$.

Proof. Since $F$ is a tensor functor, it is lax and colax. If we replace $\psi_{2}^{F}$ by $\left(\phi_{2}^{F}\right)^{-1}$ in (2.5) and (2.6), both commuting diagrams are equivalent to (2.1), which holds because $F$ is lax. Thus $F$ is a Frobenius functor.

The converse statement does not hold. For example, the functor $R$ which we define in Sect. 2.4 is Frobenius but not tensor.

Let us recall the notion of adjunctions and adjoint functors [Ma, Ch. IV.1].

Definition 2.6. An adjunction from $\mathcal{C}_{1}$ to $\mathcal{C}_{2}$ is a triple $\langle F, G, \chi\rangle$, where $F$ and $G$ are functors

$$
F: \mathcal{C}_{1} \rightarrow \mathcal{C}_{2}, \quad G: \mathcal{C}_{2} \rightarrow \mathcal{C}_{1}
$$

and $\chi$ is a natural isomorphism which assigns to each pair of objects $A_{1} \in \mathcal{C}_{1}, A_{2} \in \mathcal{C}_{2}$ a bijective map

$$
\chi_{A_{1}, A_{2}}: \operatorname{Hom}_{\mathcal{C}_{2}}\left(F\left(A_{1}\right), A_{2}\right) \stackrel{\cong}{\longrightarrow} \operatorname{Hom}_{\mathcal{C}_{1}}\left(A_{1}, G\left(A_{2}\right)\right),
$$

which is natural in both $A_{1}$ and $A_{2} . F$ is called a left-adjoint of $G$ and $G$ is called a right-adjoint of $F$.

For simplicity, we will often abbreviate $\chi_{A_{1}, A_{2}}$ as $\chi$. Associated to each adjunction $\langle F, G, \chi\rangle$, there are two natural transformations $\operatorname{id}_{\mathcal{C}_{1}} \stackrel{\delta}{\rightarrow} G F$ and $F G \stackrel{\rho}{\rightarrow} \operatorname{id}_{\mathcal{C}_{2}}$, where $\operatorname{id}_{\mathcal{C}_{1}}$ and $\operatorname{id}_{\mathcal{C}_{2}}$ are identity functors, given by

$$
\delta_{A_{1}}=\chi\left(\operatorname{id}_{F\left(A_{1}\right)}\right), \quad \rho_{A_{2}}=\chi^{-1}\left(\operatorname{id}_{G\left(A_{2}\right)}\right)
$$

for $A_{i} \in \mathcal{C}_{i}, i=1,2$. They satisfy the following two identities:

$$
G \stackrel{\delta G}{\longrightarrow} G F G \stackrel{G \rho}{\longrightarrow} G=G \stackrel{\operatorname{id}_{G}}{\longrightarrow} G, \quad F \stackrel{F \delta}{\longrightarrow} F G F \stackrel{\rho F}{\longrightarrow} F=F \stackrel{\mathrm{id}_{F}}{\longrightarrow} F .
$$

We have, for $g: F\left(A_{1}\right) \rightarrow A_{2}$ and $f: A_{1} \rightarrow G\left(A_{2}\right)$,

$$
\chi(g)=G(g) \circ \delta_{A_{1}}, \quad \chi^{-1}(f)=\rho_{A_{2}} \circ F(f) .
$$

For simplicity, $\delta_{A_{1}}$ and $\rho_{A_{2}}$ are often abbreviated as $\delta$ and $\rho$, respectively.

Let $\langle F, G, \chi\rangle$ be an adjunction from a tensor category $\mathcal{C}_{1}$ to a tensor category $\mathcal{C}_{2}$ and $\left(F, \psi_{2}^{F}, \psi_{0}^{F}\right)$ a colax tensor functor from $\mathcal{C}_{1}$ to $\mathcal{C}_{2}$. We can define a morphism 
$\phi_{0}^{G}: \mathbf{1}_{1} \rightarrow G\left(\mathbf{1}_{2}\right)$ and a natural transformation $\phi_{2}^{G}: \otimes \circ(G \times G) \rightarrow G \circ \otimes$ by, for $A, B \in \mathcal{C}_{2}$,

$$
\begin{aligned}
\phi_{0}^{G}= & \chi\left(\psi_{0}^{F}\right)=\mathbf{1}_{1} \stackrel{\delta_{\mathbf{1}_{1}}}{\longrightarrow} G F\left(\mathbf{1}_{1}\right) \stackrel{G\left(\psi_{0}^{F}\right)}{\longrightarrow} G\left(\mathbf{1}_{2}\right), \\
\phi_{2}^{G}= & \chi\left(\left(\rho_{A} \otimes \rho_{B}\right) \circ \psi_{2}^{F}\right)=G(A) \otimes G(B) \stackrel{\delta}{\rightarrow} G F(G(A) \otimes G(B)) \\
& \stackrel{G\left(\psi_{2}^{F}\right)}{\longrightarrow} G(F G(A) \otimes F G(B)) \stackrel{G\left(\rho_{A} \otimes \rho_{B}\right)}{\longrightarrow} G(A \otimes B),
\end{aligned}
$$

where we have used the first identity in (2.9). Notice that $\phi_{2}^{G}$ is natural because it is a composition of natural transformations. One can easily show that $\psi_{0}^{F}$ and $\psi_{2}^{F}$ can be re-obtained from $\phi_{0}^{G}$ and $\phi_{2}^{G}$ as follows:

$$
\begin{aligned}
\psi_{0}^{F}= & \chi^{-1}\left(\phi_{0}^{G}\right)=F\left(\mathbf{1}_{1}\right) \stackrel{F \phi_{0}^{G}}{\longrightarrow} F G\left(\mathbf{1}_{2}\right) \stackrel{\rho}{\rightarrow} \mathbf{1}_{2}, \\
\psi_{2}^{F}= & \chi^{-1}\left(\phi_{2}^{G} \circ(\delta \otimes \delta)\right)=F(U \otimes V) \stackrel{F(\delta \otimes \delta)}{\longrightarrow} F(G F(U) \otimes G F(V)) \\
& \stackrel{F \phi_{2}^{G}}{\longrightarrow} F G(F(U) \otimes F(V)) \stackrel{\rho}{\rightarrow} F(U) \otimes F(V) .
\end{aligned}
$$

for $U, V \in \mathcal{C}_{1}$. The following result is standard; for the sake of completeness, we give a proof in Appendix A.1.

Lemma 2.7. $\left(F, \psi_{2}^{F}, \psi_{0}^{F}\right)$ is a colax tensor functor iff $\left(G, \phi_{2}^{G}, \phi_{0}^{G}\right)$ is a lax tensor functor.

2.2. Algebras in tensor categories. An algebra in a tensor category $\mathcal{C}$, or a $\mathcal{C}$-algebra, is a triple $A=(A, m, \eta)$, where $A$ is an object of $\mathcal{C}, m$ (the multiplication) is a morphism $A \otimes A \rightarrow A$ such that $m \circ\left(m \otimes \mathrm{id}_{A}\right) \circ \alpha_{A, A, A}=m \circ\left(\mathrm{id}_{A} \otimes m\right)$, and $\eta$ (the unit) is a morphism $1 \rightarrow A$ such that $m \circ\left(\operatorname{id}_{A} \otimes \eta\right)=\mathrm{id}_{A} \circ r_{A}$ and $m \circ\left(\eta \otimes \mathrm{id}_{A}\right)=\mathrm{id}_{A} \circ l_{A}$. If $\mathcal{C}$ is braided and $m \circ c_{A, A}=m$, then $A$ is called commutative.

A left $A$-module is a pair $\left(M, m_{M}\right)$, where $M \in \mathcal{C}$ and $m_{M}$ is a morphism $A \otimes M \rightarrow M$ such that $m_{M} \circ\left(\operatorname{id}_{A} \otimes m_{M}\right)=m_{M} \circ\left(m_{A} \otimes \mathrm{id}_{M}\right) \circ \alpha_{A, A, M}$ and $m_{M} \circ\left(\eta_{A} \otimes \mathrm{id}_{M}\right)=\mathrm{id}_{M} \circ l_{M}$. Right $A$-modules and $A$-bimodules are defined similarly.

Definition 2.8. Let $\mathcal{C}$ be a tensor category and let $A$ be an algebra in $\mathcal{C}$.

(i) A is called simple iff it is simple as a bimodule over itself.

Let $\mathcal{C}$ be in addition $\mathbb{k}$-linear, for $\mathbb{k}$ a field.

(ii) A is called absolutely simple iff the space of A-bimodule maps from A to itself is one-dimensional, $\operatorname{dim}_{\mathbb{R}} \operatorname{Hom}_{A \mid A}(A, A)=1$.

(iii) $A$ is called haploid iff $\operatorname{dim}_{\mathbb{k}} \operatorname{Hom}(\mathbf{1}, A)=1$ [FS, Def. 4.3].

In the following we will assume that all tensor categories are strict to avoid spelling out associators and unit constraints.

A $\mathcal{C}$-coalgebra $A=(A, \Delta, \varepsilon)$ is defined analogously to a $\mathcal{C}$-algebra, i.e. $\Delta: A \rightarrow$ $A \otimes A$ and $\varepsilon: A \rightarrow \mathbf{1}$ obey coassociativity and counit conditions.

If $\mathcal{C}$ is braided and if $A$ and $B$ are $\mathcal{C}$-algebras, there are two in general non-isomorphic algebra structures on $A \otimes B$. We choose $A \otimes B$ to be the $\mathcal{C}$-algebra with multiplication $m_{A \otimes B}=\left(m_{A} \otimes m_{B}\right) \circ\left(\operatorname{id}_{A} \otimes c_{A, B}^{-1} \otimes \mathrm{id}_{B}\right)$ and unit $\eta_{A \otimes B}=\eta_{A} \otimes \eta_{B}$. Similarly, if $A$ and $B$ are $\mathcal{C}$-coalgebras, then $A \otimes B$ becomes a $\mathcal{C}$-coalgebra if we choose the comultiplication $\Delta_{A \otimes B}=\left(\operatorname{id}_{A} \otimes c_{A, B} \otimes \operatorname{id}_{B}\right) \circ\left(\Delta_{A} \otimes \Delta_{B}\right)$ and the counit $\varepsilon_{A \otimes B}=\varepsilon_{A} \otimes \varepsilon_{B}$. 
Definition 2.9. $A$ Frobenius algebra $A=(A, m, \eta, \Delta, \varepsilon)$ is an algebra and a coalgebra such that the coproduct is an intertwiner of A-bimodules,

$$
\left(\mathrm{id}_{A} \otimes m\right) \circ\left(\Delta \otimes \mathrm{id}_{A}\right)=\Delta \otimes m=\left(m \otimes \mathrm{id}_{A}\right) \circ\left(\mathrm{id}_{A} \otimes \Delta\right) .
$$

We will use the following graphical representation for the morphisms of a Frobenius algebra,

$$
m=\bigcap_{A}^{A}, \quad \eta=\prod_{A}^{A}, \Delta=\overbrace{A}^{A}, \varepsilon=\eta_{A}^{A} \text {. }
$$

A Frobenius algebra $A$ in a $\mathbb{k}$-linear tensor category, for $\mathbb{k}$ a field, is called special iff $m \circ \Delta=\zeta \operatorname{id}_{A}$ and $\varepsilon \circ \eta=\xi \operatorname{id}_{\mathbf{1}}$ for nonzero constants $\zeta, \xi \in \mathbb{k}$. If $\zeta=1$ we call $A$ normalised-special. A Frobenius algebra homomorphism between two Frobenius algebras is both an algebra homomorphism and a coalgebra homomorphism.

A (strictly) sovereign tensor category is a tensor category equipped with a left and a right duality which agree on objects and morphisms (see e.g. [Bi,FS] for more details). We will write the dualities as

$$
\begin{array}{ll}
\bigcap_{U^{\vee}}^{\rightarrow}=d_{U}: U^{\vee} \otimes U \rightarrow \mathbf{1}, & \left.\bigcap_{U}\right)_{U^{\vee}}=\tilde{d}_{U}: U \otimes U^{\vee} \rightarrow \mathbf{1}, \\
\bigcup_{U^{\vee}}=b_{U}: \mathbf{1} \rightarrow U \otimes U^{\vee}, & \bigcup_{U^{\vee}}^{U}=\tilde{b}_{U}: \mathbf{1} \rightarrow U^{\vee} \otimes U .
\end{array}
$$

In terms of these we define the left and right dimension of an object $U$ as

$$
\operatorname{dim}_{l} U=d_{U} \circ \tilde{b}_{U} \quad, \quad \operatorname{dim}_{r} U=\tilde{d}_{U} \circ b_{U},
$$

both of which are elements of $\operatorname{Hom}(\mathbf{1}, \mathbf{1})$.

Let now $\mathcal{C}$ be a sovereign tensor category. For a Frobenius algebra $A$ in $\mathcal{C}$, we define two morphisms:

$$
\Phi_{A}=\int_{A}^{9} \Phi_{A}^{A^{\vee}}, \quad \underbrace{A^{\vee}}_{A}
$$

Definition 2.10. A Frobenius algebra $A$ is symmetric iff $\Phi_{A}=\Phi_{A}^{\prime}$.

The following lemma shows that under certain conditions we do not need to distinguish the various notions of simplicity in Definition 2.8.

Lemma 2.11. Let $A$ be a commutative symmetric Frobenius algebra in a $\mathbb{C}$-linear semisimple sovereign braided tensor category $\mathcal{C}$ and suppose that $\operatorname{dim}_{l} A \neq 0$. Then the following are equivalent. 
(i) A is simple.

(ii) $A$ is absolutely simple.

(iii) A is haploid.

Proof. (ii) $\Leftrightarrow$ (iii): $A$ is haploid iff it is absolutely simple as a left module over itself [FS, Eq. (4.17)]. Furthermore, for a commutative algebra we have $\operatorname{Hom}_{A}(A, A)=$ $\operatorname{Hom}_{A \mid A}(A, A)$, and so $A$ is haploid iff it is absolutely simple.

(i) $\Rightarrow$ (ii): If $A$ is simple, then every nonzero element of $\operatorname{Hom}_{A \mid A}(A, A)$ is invertible. Hence this space forms a division algebra over $\mathbb{C}$, and is therefore isomorphic to $\mathbb{C}$.

(iii) $\Rightarrow(\mathrm{i})$ : Since $\mathcal{C}$ is semi-simple and $A$ is haploid, also $\operatorname{Hom}(A, \mathbf{1})$ is one-dimensional. The counit $\varepsilon$ is a nonzero element in this space, and so gives a basis. This implies firstly, that $\varepsilon \circ \eta \neq 0$, and secondly, that there is a constant $\beta \in \mathbb{C}$ such that

$$
\beta \cdot \varepsilon=d_{A} \circ\left(\operatorname{id}_{A^{\vee}} \otimes m\right) \circ\left(\tilde{b}_{A} \otimes \mathrm{id}_{A}\right) .
$$

Composing with $\eta$ from the right yields $\beta \varepsilon \circ \eta=\operatorname{dim}_{l} A$. The right-hand side is nonzero, and so $\beta \neq 0$. By [FRS, Lem. 3.11], $A$ is special. We have already proved (ii) $\Leftrightarrow$ (iii), and so $A$ is absolutely simple. A special Frobenius algebra in a semi-simple category has a semi-simple category of bimodules (apply [FS, Prop. 5.24] to the algebra tensored with its opposite algebra). For semi-simple $\mathbb{C}$-linear categories, simple and absolutely simple are equivalent. ${ }^{2}$ Thus $A$ is simple.

Remark 2.12. For a Frobenius algebra $A$ the morphisms (2.15) are invertible, and hence $A \cong A^{\vee}$. In this case one has $\operatorname{dim}_{l} A=\operatorname{dim}_{r} A$ [FS, Rem.3.6.3] and so we could have stated the above lemma equivalently with the condition $\operatorname{dim}_{r} A \neq 0$.

Let $F: \mathcal{C}_{1} \rightarrow \mathcal{C}_{2}$ be a lax tensor functor between two tensor categories $\mathcal{C}_{1}, \mathcal{C}_{2}$ and let $\left(A, m_{A}, \eta_{A}\right)$ be an algebra in $\mathcal{C}_{1}$. Define morphisms $F(A) \otimes F(A) \stackrel{m_{F(A)}}{\longrightarrow} F(A)$ and $\mathbf{1}_{2} \stackrel{\eta_{F(A)}}{\longrightarrow} F(A)$ as

$$
m_{F(A)}=F\left(m_{A}\right) \circ \phi_{2}^{F}, \quad \eta_{F(A)}=F\left(\eta_{A}\right) \circ \phi_{0}^{F} .
$$

Then $\left(F(A), m_{F(A)}, \eta_{F(A)}\right)$ is an algebra in $\mathcal{C}_{2}$ [JS, Prop.5.5]. If $f: A \rightarrow B$ is an algebra homomorphism between two algebras $A, B \in \mathcal{C}_{1}$, then $F(f): F(A) \rightarrow F(B)$ is also an algebra homomorphism. If $\left(M, m_{M}\right)$ is a left (or right) $A$-module in $\mathcal{C}_{1}$, then $\left(F(M), F\left(m_{M}\right) \circ \phi_{2}^{F}\right)$ is a left (or right) $F(A)$-module; if $M$ has a $A$-bimodule structure, then $F(M)$ naturally has a $F(A)$-bimodule structure.

Similarly, if $\left(A, \Delta_{A}, \varepsilon_{A}\right)$ is a coalgebra in $\mathcal{C}_{1}$ and $F: \mathcal{C}_{1} \rightarrow \mathcal{C}_{2}$ is a colax tensor functor, then $F(A)$ with coproduct $F(A) \stackrel{\Delta_{F(A)}}{\longrightarrow} F(A) \otimes F(A)$ and counit $F(A) \stackrel{\varepsilon_{F(A)}}{\longrightarrow} \mathbf{1}_{2}$ given by

$$
\Delta_{F(A)}=\psi_{2}^{F} \circ F\left(\Delta_{A}\right), \quad \varepsilon_{F(A)}=\psi_{0}^{F} \circ F\left(\varepsilon_{A}\right),
$$

is a coalgebra in $\mathcal{C}_{2}$. If $f: A \rightarrow B$ is a coalgebra homomorphism between two coalgebras $A, B \in \mathcal{C}_{1}$, then $F(f): F(A) \rightarrow F(B)$ is also a coalgebra homomorphism.

2 To see this note that if $U$ is simple, then the $\mathbb{C}$-vector space $\operatorname{Hom}(U, U)$ is a division algebra, and hence $\operatorname{Hom}(U, U)=\mathbb{C} \operatorname{id}_{U}$. Conversely, if $U$ is not simple, then $U=U_{1} \oplus U_{2}$ and $\operatorname{Hom}(U, U)$ contains at least two linearly independent elements, namely $\operatorname{id}_{U_{1}}$ and $\operatorname{id}_{U_{2}}$. 
Proposition 2.13. ${ }^{3}$ If $F: \mathcal{C}_{1} \rightarrow \mathcal{C}_{2}$ is a Frobenius functor and $\left(A, m_{A}, \eta_{A}, \Delta_{A}, \varepsilon_{A}\right) a$ Frobenius algebra in $\mathcal{C}_{1}$, then $\left(F(A), m_{F(A)}, \eta_{F(A)}, \Delta_{F(A)}, \varepsilon_{F(A)}\right)$ is a Frobenius algebra in $\mathcal{C}_{2}$.

Proof. One Frobenius property, $\left(m_{F(A)} \otimes \mathrm{id}_{F(A)}\right) \circ\left(\mathrm{id}_{F(A)} \otimes \Delta_{F(A)}\right)=\Delta_{F(A)} \circ m_{F(A)}$, follows from the commutativity of the following diagram (we spell out the associativity isomorphisms):

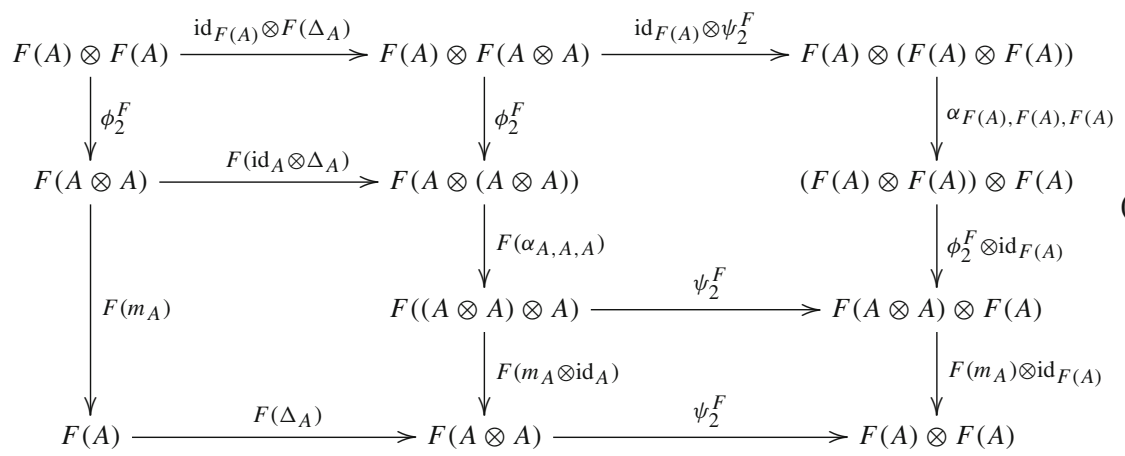

The commutativity of the upper-left subdiagram follows from the naturalness of $\phi_{2}^{F}$, that of the upper-right subdiagram follows from (2.5), that of the lower-left subdiagram follows from the Frobenius properties of $A$, and that of the lower-right subdiagram follows from the naturalness of $\psi_{2}^{F}$. The proof of the other Frobenius property is similar.

Proposition 2.14. If $F: \mathcal{C}_{1} \rightarrow \mathcal{C}_{2}$ is a tensor functor and $A$ a Frobenius algebra in $\mathcal{C}_{1}$, then:

(i) $F(A)$ has a natural structure of Frobenius algebra as given in Proposition 2.13;

(ii) If $A$ is (normalised-)special, so is $F(A)$.

Proof. Part (i) follows from Propositions 2.5 and 2.13. Part (ii) is a straightforward verification of the definition, using $\psi_{2}^{F}=\left(\phi_{2}^{F}\right)^{-1}$ and $\psi_{0}^{F}=\left(\phi_{0}^{F}\right)^{-1}$.

Let $\mathcal{C}_{1}, \mathcal{C}_{2}$ be sovereign tensor categories and $F: \mathcal{C}_{1} \rightarrow \mathcal{C}_{2}$ a Frobenius functor. We define two morphisms $I_{F\left(A^{\vee}\right)}, I_{F\left(A^{\vee}\right)}^{\prime}: F\left(A^{\vee}\right) \rightarrow F(A)^{\vee}$, for a Frobenius algebra $A$ in $\mathcal{C}_{1}$, as follows:

$$
\begin{aligned}
& I_{F\left(A^{\vee}\right)}=\left(\left(\psi_{0}^{F} \circ F\left(d_{A}\right) \circ \phi_{2}^{F}\right) \otimes \operatorname{id}_{F(A)^{\vee}}\right) \circ\left(\operatorname{id}_{F\left(A^{\vee}\right)} \otimes b_{F(A)}\right), \\
& I_{F\left(A^{\vee}\right)}^{\prime}=\left(\operatorname{id}_{F(A)^{\vee}} \otimes\left(\psi_{0}^{F} \circ F\left(\tilde{d}_{A}\right) \circ \phi_{2}^{F}\right)\right) \circ\left(\tilde{b}_{F(A)} \otimes \operatorname{id}_{F\left(A^{\vee}\right)}\right) .
\end{aligned}
$$

It is easy to see that these are isomorphisms.

Lemma 2.15. If $F: \mathcal{C}_{1} \rightarrow \mathcal{C}_{2}$ is a Frobenius functor and $A$ a Frobenius algebra in $\mathcal{C}_{1}$, then

$$
\Phi_{F(A)}=I_{F\left(A^{\vee}\right)} \circ F\left(\Phi_{A}\right), \quad \Phi_{F(A)}^{\prime}=I_{F\left(A^{\vee}\right)}^{\prime} \circ F\left(\Phi_{A}^{\prime}\right) .
$$

3 After the preprint of the present paper appeared we noticed that this proposition is also proved in [DP, Cor. 5]. 
Proof. We only prove the first equality, the second one can be seen in the same way. By definition, we have

$$
\begin{aligned}
& I_{F\left(A^{\vee}\right)} \circ F\left(\Phi_{A}\right) \\
& =\left(\left(\psi_{0}^{F} \circ F\left(d_{A}\right) \circ \phi_{2}^{F}\right) \otimes \operatorname{id}_{F(A)^{\vee}}\right) \circ\left(\operatorname{id}_{F\left(A^{\vee}\right)} \otimes b_{F(A)}\right) \circ F\left(\Phi_{A}\right) \\
& =\left\{\left[\left(\psi_{0}^{F} \circ F\left(d_{A}\right) \circ \phi_{2}^{F}\right) \circ\left(F\left(\Phi_{A}\right) \otimes \operatorname{id}_{F(A)}\right)\right] \otimes \operatorname{id}_{F(A)}\right\} \circ\left(\operatorname{id}_{F(A)} \otimes b_{F(A)}\right) \text {. }
\end{aligned}
$$

For the term inside the square brackets we find

$$
\begin{gathered}
\psi_{0}^{F} \circ F\left(d_{A}\right) \circ \phi_{2}^{F} \circ\left(F\left(\Phi_{A}\right) \otimes \operatorname{id}_{F(A)}\right)=\psi_{0}^{F} \circ F\left(d_{A}\right) \circ F\left(\Phi_{A} \otimes \mathrm{id}_{A}\right) \circ \phi_{2}^{F} \\
=\psi_{0}^{F} \circ F\left(d_{A} \circ\left(\Phi_{A} \otimes \mathrm{id}_{A}\right)\right) \circ \phi_{2}^{F}=\psi_{0}^{F} \circ F\left(\varepsilon_{A} \circ m_{A}\right) \circ \phi_{2}^{F} .
\end{gathered}
$$

On the other hand, by definition, $\Phi_{F(A)}=\left[\left(\left(\psi_{0}^{F} \circ F\left(\varepsilon_{A}\right) \circ\left(F\left(m_{A}\right) \circ \phi_{2}^{F}\right)\right) \otimes \mathrm{id}_{F(A)^{\vee}}\right] \circ\right.$ $\left(\operatorname{id}_{F(A)} \otimes b_{F(A)}\right)$. This demonstrates the first equality in (2.21).

Proposition 2.16. Let $F: \mathcal{C}_{1} \rightarrow \mathcal{C}_{2}$ be a tensor functor, $G: \mathcal{C}_{2} \rightarrow \mathcal{C}_{1}$ a functor, $\langle F, G, \chi\rangle$ an adjunction, $A$ a $\mathcal{C}_{1}$-algebra, and $B$ a $\mathcal{C}_{2}$-algebra. Then $f: A \rightarrow G(B)$ is an algebra homomorphism if and only if $\tilde{f}=\chi^{-1}(f): F(A) \rightarrow B$ is an algebra homomorphism.

Proof. We need to show that

$$
m_{G(B)} \circ(f \otimes f)=f \circ m_{A} \text { and } f \circ \eta_{A}=\eta_{G(B)},
$$

is equivalent to

$$
m_{B} \circ(\tilde{f} \otimes \tilde{f})=\tilde{f} \circ m_{F(A)} \text { and } \tilde{f} \circ \eta_{F(A)}=\eta_{B} s .
$$

We first prove that the first identity in (2.23) is equivalent to the first identity in (2.24). For the left-hand side of the first identity in (2.23) we have the following equalities:

$$
\begin{aligned}
m_{G(B)} \circ(f \otimes f) & \stackrel{(1)}{=} G\left(m_{B}\right) \circ \phi_{2}^{G} \circ(f \otimes f) \\
& \stackrel{(2)}{=} G\left(m_{B}\right) \circ G(\rho \otimes \rho) \circ G\left(\psi_{2}^{F}\right) \circ \delta \circ(f \otimes f) \\
& \stackrel{(3)}{=} G\left(m_{B}\right) \circ G(\rho \otimes \rho) \circ G\left(\psi_{2}^{F}\right) \circ G F(f \otimes f) \circ \delta \\
& \stackrel{(4)}{=} G\left(m_{B}\right) \circ G(\rho \otimes \rho) \circ G(F(f) \otimes F(f)) \circ G\left(\psi_{2}^{F}\right) \circ \delta \\
& \stackrel{(5)}{=} G\left(m_{B} \circ(\rho \otimes \rho) \circ(F(f) \otimes F(f)) \circ \psi_{2}^{F}\right) \circ \delta \\
& \stackrel{(6)}{=} \chi\left(m_{B} \circ(\rho \otimes \rho) \circ(F(f) \otimes F(f)) \circ \psi_{2}^{F}\right),
\end{aligned}
$$

where (1) is the definition of $m_{G(B)}$ in (2.17), (2) is the second identity in (2.10), (3) and (4) are naturality of $\delta$ and $\psi_{2}^{F}$, respectively, step (5) is functoriality of $G$ and finally step (6) is (2.9). For the right hand side of the first identity in (2.23) we get

$$
\begin{aligned}
f \circ m_{A} & \stackrel{(1)}{=} G \rho \circ \delta G \circ\left(f \circ m_{A}\right) \\
& \stackrel{(2)}{=} G \rho \circ G F\left(f \circ m_{A}\right) \circ \delta \\
& \stackrel{(3)}{=} G\left(\rho \circ F\left(f \circ m_{A}\right)\right) \circ \delta \\
& \stackrel{(4)}{=} \chi\left(\rho \circ F(f) \circ F\left(m_{A}\right)\right),
\end{aligned}
$$


where (1) is the adjunction property (2.8), (2) is naturality of $\delta,(3)$ functoriality of $G$, and (4) amounts to (2.9) and functoriality of $F$.

On the other hand, we see that the first equality in (2.24) is equivalent to

$$
m_{B} \circ(\rho \otimes \rho) \circ(F(f) \otimes F(f))=\rho \circ F(f) \circ F\left(m_{A}\right) \circ \phi_{2}^{F} .
$$

Using that $\phi_{2}^{F}$ is invertible with inverse $\left(\phi_{2}^{F}\right)^{-1}=\psi_{2}^{F}$ and that $\chi$ is an isomorphism, it follows that the statement that $(2.25)$ is equal to $(2.26)$ is equivalent to the identity (2.27).

Now we prove that the second identity in (2.23) is equivalent to the second identity in (2.24). Using (2.17) and (2.10) we can write $\eta_{G(B)}=G\left(\eta_{B}\right) \circ \phi_{0}^{F}=G\left(\eta_{B}\right) \circ G\left(\psi_{0}^{F}\right) \circ \delta_{\mathbf{1}}$. Together with (2.9) this shows that the second identity in (2.23) is equivalent to

$$
f \circ \eta_{A}=\chi\left(\eta_{B} \circ \psi_{0}^{F}\right) .
$$

On the other hand, the second identity in (2.24) is equivalent to

$$
\rho \circ F(f) \circ F\left(\eta_{A}\right) \circ \phi_{0}^{F}=\eta_{B},
$$

which, by $\phi_{0}^{F}=\left(\psi_{0}^{F}\right)^{-1}$ and (2.9), is further equivalent to (2.28).

Definition 2.17. Let $\left(A, m_{A}, \eta_{A}, \Delta_{A}, \varepsilon_{A}\right)$ and $\left(B, m_{B}, \eta_{B}, \Delta_{B}, \varepsilon_{B}\right)$ be two Frobenius algebras in a tensor category $\mathcal{C}$. For $f: A \rightarrow B$, we define $f^{*}: B \rightarrow A$ by

$$
f^{*}=\left(\left(\varepsilon_{B} \circ m_{B}\right) \otimes \mathrm{id}_{A}\right) \circ\left(\mathrm{id}_{B} \otimes f \otimes \mathrm{id}_{A}\right) \circ\left(\mathrm{id}_{B} \otimes\left(\Delta_{A} \circ \eta_{A}\right)\right) .
$$

The following lemma is immediate from the definition of $(\cdot)^{*}$ and the properties of Frobenius algebras. We omit the proof.

Lemma 2.18. Let $\mathcal{C}$ be a tensor category, let $A, B, C$ be Frobenius algebras in $\mathcal{C}$, and let $f: A \rightarrow B$ and $g: B \rightarrow C$ be morphisms.

(i) $(g \circ f)^{*}=f^{*} \circ g^{*}$.

(ii) $f$ is a monomorphism iff $f^{*}$ is an epimorphism.

(iii) $f$ is an algebra map iff $f^{*}$ is a coalgebra map.

(iv) If $f$ is a homomorphism of Frobenius algebras, then $f^{*} \circ f=\mathrm{id}_{A}$ and $f \circ f^{*}=$ $\operatorname{id}_{B}$.

(v) If $\mathcal{C}$ is sovereign and if $A$ and $B$ are symmetric, then $f^{* *}=f$.

Let $\mathcal{C}$ and $\mathcal{D}$ be tensor categories and let $F: \mathcal{C} \rightarrow \mathcal{D}$ be a Frobenius functor. Given Frobenius algebras $A, B$ in $\mathcal{C}$ and a morphism $f: A \rightarrow B$, the next lemma shows how $(\cdot)^{*}$ behaves under $F$.

Lemma 2.19. $F\left(f^{*}\right)=F(f)^{*}$.

Proof. The definition of the structure morphisms of the Frobenius algebra $F(A)$ is given in (2.17) and (2.18). Substituting these definitions gives

$$
\begin{aligned}
F(f)^{*}= & {\left[\left(\psi_{0}^{F} \circ F\left(\varepsilon_{B}\right) \circ F\left(m_{B}\right) \circ \phi_{2}^{F}\right) \otimes \operatorname{id}_{F(A)}\right] \circ\left[\operatorname{id}_{F(B)} \otimes F(f) \otimes \operatorname{id}_{F(A)}\right] } \\
& \circ\left[\operatorname{id}_{F(B)} \otimes\left(\psi_{2}^{F} \circ F\left(\Delta_{A}\right) \circ F\left(\eta_{A}\right) \circ \phi_{0}^{F}\right)\right] \\
= & \left(\psi_{0}^{F} \otimes \operatorname{id}_{F(A)}\right) \circ\left[F\left(\varepsilon_{B} \circ m_{B} \circ\left(\operatorname{id}_{B} \otimes f\right)\right) \otimes \operatorname{id}_{F(A)}\right] \\
& \circ\left(\phi_{2}^{F} \otimes \operatorname{id}_{F(A)}\right) \circ\left(\operatorname{id}_{F(B)} \otimes \psi_{2}^{F}\right) \\
& \circ\left[\operatorname{id}_{F(B)} \otimes F\left(\Delta_{A} \circ \eta_{A}\right)\right] \circ\left(\operatorname{id}_{F(B)} \otimes \phi_{0}^{F}\right) .
\end{aligned}
$$


In the middle line of the last expression we can use the defining property (2.5) of $F$, namely we substitute $\left(\phi_{2}^{F} \otimes \operatorname{id}_{F(A)}\right) \circ\left(\operatorname{id}_{F(B)} \otimes \psi_{2}^{F}\right)=\psi_{2}^{F} \circ \phi_{2}^{F}$. Then $\psi_{2}^{F}$ can be moved to the left, and $\phi_{2}^{F}$ to the right, until they can be omitted against $\psi_{0}^{F}$ and $\phi_{0}^{F}$, respectively, using (2.2) and (2.4). This results in

$$
F(f)^{*}=F\left(\left(\varepsilon_{B} \circ m_{B} \circ\left(\operatorname{id}_{B} \otimes f\right)\right) \otimes \operatorname{id}_{A}\right) \circ F\left(\operatorname{id}_{B} \otimes\left(\Delta_{A} \circ \eta_{A}\right)\right),
$$

which is nothing but $F\left(f^{*}\right)$.

2.3. Modular tensor categories. Let $\mathcal{C}$ be a modular tensor category [T,BK], i.e. an abelian semi-simple finite $\mathbb{C}$-linear ribbon category with simple tensor unit $\mathbf{1}$ and a nondegeneracy condition on the braiding (to be stated in a moment). We denote the set of equivalence classes of simple objects in $\mathcal{C}$ by $\mathcal{I}$, elements in $\mathcal{I}$ by $i, j, k \in \mathcal{I}$ and their representatives by $U_{i}, U_{j}, U_{k}$. We also set $U_{0}=1$ and for an index $k \in \mathcal{I}$ we define $\bar{k}$ by $U_{\bar{k}} \cong U_{k}^{\vee}$.

Since the tensor unit is simple, we shall for modular tensor categories identify $\operatorname{Hom}(\mathbf{1}, \mathbf{1}) \cong \mathbb{C}($ cf. footnote 2$)$. Define numbers $s_{i, j} \in \mathbb{C}$ by ${ }^{4}$

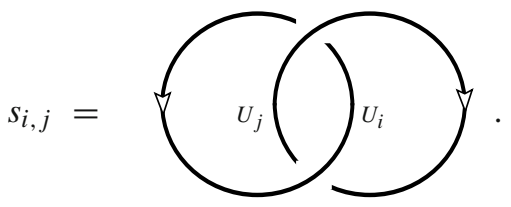

They obey $s_{i, j}=s_{j, i}$ and $s_{0, i}=\operatorname{dim} U_{i}$, see e.g. [BK, Sect.3.1]. (In a ribbon category the left and right dimension (2.14) of $U_{i}$ coincide and are denoted by $\operatorname{dim} U_{i}$.) The non-degeneracy condition on the braiding of a modular tensor category is that the $|\mathcal{I}| \times|\mathcal{I}|$-matrix $s$ should be invertible. In fact [BK, Thm. 3.1.7],

$$
\sum_{k \in \mathcal{I}} s_{i k} s_{k j}=\operatorname{Dim} \mathcal{C} \delta_{i, \bar{j}}
$$

where $\operatorname{Dim} \mathcal{C}=\sum_{i \in \mathcal{I}}\left(\operatorname{dim} U_{i}\right)^{2}$. One can show (even in the weaker context of fusion categories over $\mathbb{C}$ ) that $\operatorname{Dim} \mathcal{C} \geq 1$ [ENO1, Thm. 2.3]. In particular, $\operatorname{Dim} \mathcal{C} \neq 0$. We fix once and for all a square $\operatorname{root} \sqrt{\operatorname{Dim} \mathcal{C}}$ of $\operatorname{Dim} \mathcal{C}$.

Let us fix a basis $\left\{\lambda_{(i, j) k}^{\alpha}\right\}_{\alpha=1}^{N_{i j}^{k}}$ in $\operatorname{Hom}_{\mathcal{C}}\left(U_{i} \otimes U_{j}, U_{k}\right)$ and the dual basis $\left\{\Upsilon_{\alpha}^{(i, j) k}\right\}_{\alpha=1}^{N_{i j}^{k}}$ in $\operatorname{Hom}_{\mathcal{C}}\left(U_{k}, U_{i} \otimes U_{j}\right)$. The duality of the bases means that $\lambda_{(i, j) k}^{\alpha} \circ \Upsilon_{\beta}^{(i, j) k}=\delta_{\alpha, \beta} \operatorname{id}_{U_{k}}$. We also fix $\lambda_{(0, i) i}=\lambda_{(i, 0) i}=\mathrm{id}_{U_{i}}$. We denote the basis vectors graphically as follows:

$$
\lambda_{(i, j) k}^{\alpha}=\overbrace{U_{i}}^{U_{k}}, \quad \Upsilon_{\alpha}^{(i, j) k}=\bigcap_{U_{j}}^{U_{i}} .
$$

\footnotetext{
4 In the graphical notation used below, we have given an orientation to the ribbons indicated by the arrows. For example, it is understood that this orientation determines which of the duality morphisms in (2.13) to use.
} 
For $V \in \mathcal{C}$ we also choose a basis $\left\{b_{V}^{(i ; \alpha)}\right\}$ of $\operatorname{Hom}_{\mathcal{C}}\left(V, U_{i}\right)$ and the dual basis $\left\{b_{(i ; \beta)}^{V}\right\}$ of $\operatorname{Hom}_{\mathcal{C}}\left(U_{i}, V\right)$ for $i \in \mathcal{I}$ such that $b_{V}^{(i ; \alpha)} \circ b_{(i ; \beta)}^{V}=\delta_{\alpha \beta} \operatorname{id}_{U_{i}}$. We use the graphical notation

$$
b_{V}^{(i ; \alpha)}=\left.\right|_{V} ^{\alpha}, \quad b_{(i ; \alpha)}^{V}=\oint_{U_{i}}^{U_{i}} .
$$

Given two modular tensor categories $\mathcal{C}$ and $\mathcal{D}$, by $\mathcal{C} \otimes \mathcal{D}$ we mean the tensor product of additive categories over $\mathbb{C}[\mathrm{BK}$, Def. 1.1.15], i.e. the category whose objects are direct sums of pairs $V \times W$ of objects $V \in \mathcal{C}$ and $W \in \mathcal{D}$ and whose morphism spaces are

$$
\operatorname{Hom}_{\mathcal{C} \otimes \mathcal{D}}\left(V \times W, V^{\prime} \times W^{\prime}\right)=\operatorname{Hom}_{\mathcal{C}}\left(V, V^{\prime}\right) \otimes_{\mathbb{C}} \operatorname{Hom}_{\mathcal{C}}\left(W, W^{\prime}\right)
$$

for pairs, and direct sums of these if the objects are direct sums of pairs.

If we replace the braiding and the twist in $\mathcal{C}$ by the antibraiding $c^{-1}$ and the antitwist $\theta^{-1}$ respectively, we obtain another ribbon category structure on $\mathcal{C}$. In order to distinguish these two distinct structures, we denote $(\mathcal{C}, c, \theta)$ and $\left(\mathcal{C}, c^{-1}, \theta^{-1}\right)$ by $\mathcal{C}_{+}$and $\mathcal{C}_{-}$ respectively. As in the Introduction, we will abbreviate

$$
\mathcal{C}_{ \pm}^{2}=\mathcal{C}_{+} \otimes \mathcal{C}_{-}
$$

Note that a set of representatives of the simple objects in $\mathcal{C}_{ \pm}^{2}$ is given by $U_{i} \times U_{j}$ for $i, j \in \mathcal{I}$.

For the remainder of Sect. 2 we fix a modular tensor category $\mathcal{C}$.

2.4. The functors $T$ and $R$. The tensor product bifunctor $\otimes$ can be naturally extended to a functor $T: \mathcal{C}_{ \pm}^{2} \rightarrow \mathcal{C}$. Namely, $T\left(\oplus_{i=1}^{N} V_{i} \times W_{i}\right)=\oplus_{i=1}^{N} V_{i} \otimes W_{i}$ for all $V_{i}, W_{i} \in \mathcal{C}$ and $N \in \mathbb{N}$. The functor $T$ becomes a tensor functor as follows. For $\phi_{0}^{T}: \mathbf{1} \rightarrow T(\mathbf{1} \times \mathbf{1})$ take $\phi_{0}^{T}=\mathrm{id}_{\mathbf{1}}$ (or $l_{1}^{-1}$ in the non-strict case). Next notice that, for $U, V, W, X \in \mathcal{C}$,

$$
\begin{aligned}
& T(U \times V) \otimes T(W \times X)=(U \otimes V) \otimes(W \otimes X), \\
& T((U \times V) \otimes(W \times X))=(U \otimes W) \otimes(V \otimes X) .
\end{aligned}
$$

We define $\phi_{2}^{T}: T(U \times V) \otimes T(W \times X) \rightarrow T((U \times V) \otimes(W \times X))$ by

$$
\phi_{2}^{T}=\mathrm{id}_{U} \otimes c_{W V}^{-1} \otimes \mathrm{id}_{X}
$$

(In the non-strict case the appropriate associators have to be added.) The above definition of $\phi_{2}^{T}$ can be naturally extended to a morphism $\phi_{2}^{T}: T\left(M_{1} \otimes M_{2}\right) \rightarrow T\left(M_{1}\right) \otimes T\left(M_{2}\right)$ for any pair of objects $M_{1}, M_{2}$ in $\mathcal{C}_{ \pm}^{2}$. The following result can be checked by direct calculation [JS, Prop. 5.2].

Lemma 2.20. The triple $\left(T, \phi_{2}^{T}, \phi_{0}^{T}\right)$ gives a tensor functor. 
In particular, $\left(T, \phi_{2}^{T}, \phi_{0}^{T}, \psi_{2}^{T}, \psi_{0}^{T}\right)$, where $\psi_{2}^{T}=\left(\phi_{2}^{T}\right)^{-1}$ and $\psi_{0}^{T}=\left(\phi_{0}^{T}\right)^{-1}$, gives a Frobenius functor.

Define the functor $R: \mathcal{C} \rightarrow \mathcal{C}_{ \pm}^{2}$ as follows: for $A \in \mathcal{C}$ and $f \in \operatorname{Hom}_{\mathcal{C}}(A, B)$,

$$
R(A)=\bigoplus_{i \in \mathcal{I}}\left(A \otimes U_{i}^{\vee}\right) \times U_{i}, \quad R(f)=\bigoplus_{i \in \mathcal{I}}\left(f \otimes \mathrm{id}_{U_{i}^{\vee}}\right) \times \mathrm{id}_{U_{i}} .
$$

This functor was also considered in a slightly different context in [ENO2, Prop. 2.3]. The family of isomorphisms $\gamma_{A}^{R}=\oplus_{i \in \mathcal{I}} \frac{\operatorname{Dim\mathcal {C}}}{\operatorname{dim} U_{i}} \mathrm{id}_{\left(A \otimes U_{i}^{\vee}\right) \times U_{i}} \in \operatorname{Aut}(R(A))$ defines a natural isomorphism $\gamma^{R}: R \rightarrow R$.

Our next aim is to show that $R$ is left and right adjoint to $T$, in other words $R$ and $T$ form an ambidextrous adjunction (see e.g. [Ld] for a discussion of ambidextrous adjunctions). To this end we introduce two linear isomorphisms, for $A \in \mathcal{C}$ and $M \in \mathcal{C}_{ \pm}^{2}$,

$$
\begin{aligned}
& \hat{\chi}: \operatorname{Hom}_{\mathcal{C}}(T(M), A) \longrightarrow \operatorname{Hom}_{\mathcal{C}_{ \pm}^{2}}(M, R(A)), \\
& \check{\chi}: \operatorname{Hom}_{\mathcal{C}}(A, T(M)) \longrightarrow \operatorname{Hom}_{\mathcal{C}_{ \pm}^{2}}(R(A), M) .
\end{aligned}
$$

If we decompose $M$ as $M=\oplus_{n=1}^{N} M_{n}^{l} \times M_{n}^{r}$, then $\hat{\chi}$ and $\check{\chi}$ are given by
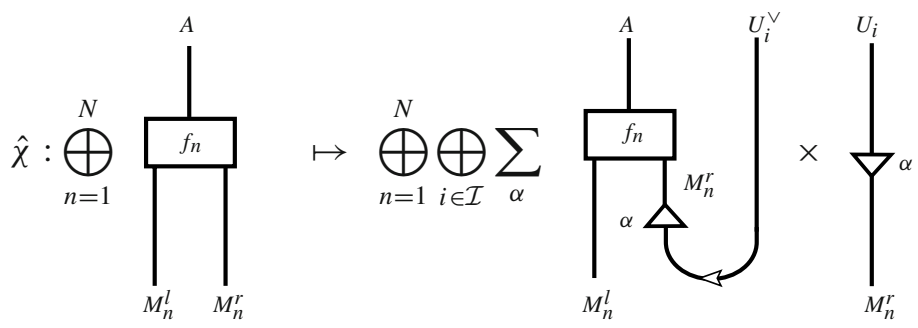

and
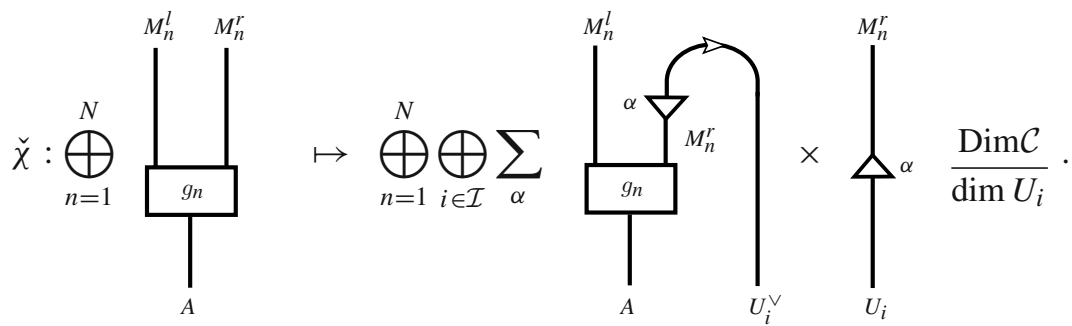

Notice that $\hat{\chi}$ and $\check{\chi}$ are independent of the choice of basis.

Theorem 2.21. $\langle T, R, \hat{\chi}\rangle$ and $\left\langle R, T, \check{\chi}^{-1}\right\rangle$ are adjunctions, i.e. $R$ is both left and right adjoint of $T$.

Proof. Write $M$ as $M=\oplus_{n=1}^{N} M_{n}^{l} \times M_{n}^{r}$. The isomorphism $\hat{\chi}$ amounts to the following composition of natural isomorphisms,

$$
\begin{aligned}
& \operatorname{Hom}_{\mathcal{C}}(T(M), A)=\oplus_{n} \operatorname{Hom}_{\mathcal{C}}\left(M_{n}^{l} \otimes M_{n}^{r}, A\right) \\
& \cong \oplus_{n, i} \operatorname{Hom}_{\mathcal{C}}\left(M_{n}^{l} \otimes U_{i}, A\right) \otimes \operatorname{Hom}_{\mathcal{C}}\left(M_{n}^{r}, U_{i}\right) \\
& \cong \oplus_{n, i} \operatorname{Hom}_{\mathcal{C}}\left(M_{n}^{l}, A \otimes U_{i}^{\vee}\right) \otimes \operatorname{Hom}_{\mathcal{C}}\left(M_{n}^{r}, U_{i}\right)=\operatorname{Hom}_{\mathcal{C}_{ \pm}^{2}}(M, R(A)) .
\end{aligned}
$$


Thus $\hat{\chi}$ is natural. Let $\left(\gamma_{A}^{R}\right)^{*}: \operatorname{Hom}_{\mathcal{C}_{ \pm}^{2}}(R(A), M) \rightarrow \operatorname{Hom}_{\mathcal{C}_{ \pm}^{2}}(R(A), M)$ denote the pull-back of $\gamma_{A}^{R}$. The isomorphism $\check{\chi}$ is equal to the composition of $\left(\gamma_{A}^{R}\right)^{*}$ and the following sequence of natural isomorphisms:

$$
\begin{aligned}
& \operatorname{Hom}_{\mathcal{C}}(A, T(M))=\oplus_{n} \operatorname{Hom}_{\mathcal{C}}\left(A, M_{n}^{l} \otimes M_{n}^{r}\right) \\
& \cong \oplus_{n, i} \operatorname{Hom}_{\mathcal{C}}\left(A, M_{n}^{l} \otimes U_{i}\right) \otimes \operatorname{Hom}_{\mathcal{C}}\left(U_{i}, M_{n}^{r}\right) \\
& \cong \oplus_{n, i} \operatorname{Hom}_{\mathcal{C}}\left(A \otimes U_{i}^{\vee}, M_{n}^{l}\right) \otimes \operatorname{Hom}_{\mathcal{C}}\left(U_{i}, M_{n}^{r}\right)=\operatorname{Hom}_{\mathcal{C}_{ \pm}^{2}}(R(A), M) .
\end{aligned}
$$

We have proved that both $\hat{\chi}$ and $\check{\chi}$ are natural isomorphisms.

There are four natural transformations associated to $\hat{\chi}$ and $\check{\chi}$, namely

$$
\operatorname{id}_{\mathcal{C}_{ \pm}^{2}} \stackrel{\hat{\delta}}{\rightarrow} R T \stackrel{\check{\rho}}{\rightarrow} \operatorname{id}_{\mathcal{C}_{ \pm}^{2}} \text { and } \operatorname{id}_{\mathcal{C}} \stackrel{\check{s}}{\rightarrow} T R \stackrel{\hat{\rho}}{\rightarrow} \operatorname{id}_{\mathcal{C}}
$$

defined by, for $A \in \mathcal{C}, M \in \mathcal{C}_{ \pm}^{2}$,

$$
\begin{array}{ll}
\hat{\delta}_{M}=\hat{\chi}\left(\operatorname{id}_{T(M)}\right), & \hat{\rho}_{A}=\hat{\chi}^{-1}\left(\operatorname{id}_{R(A)}\right), \\
\check{\rho}_{M}=\check{\chi}\left(\operatorname{id}_{T(M)}\right), & \check{\delta}_{A}=\check{\chi}^{-1}\left(\operatorname{id}_{R(A)}\right) .
\end{array}
$$

They can be expressed graphically as follows, with $M=\oplus_{n=1}^{N} M_{n}^{l} \times M_{n}^{r}$,

$$
\begin{aligned}
& \hat{\delta}_{M}=\bigoplus \sum_{n, i} \sum_{\alpha} \uparrow_{M_{n}^{l}}^{M_{n}^{l}} \alpha \int_{M_{n}^{r}}^{M_{n}^{r}} \times\left.\right|_{A} ^{U_{i}^{\vee}}, \quad \hat{\rho}_{A}=\bigoplus_{i \in \mathcal{I}}^{U_{i}} \overbrace{U_{i}^{\vee}}^{A}
\end{aligned}
$$

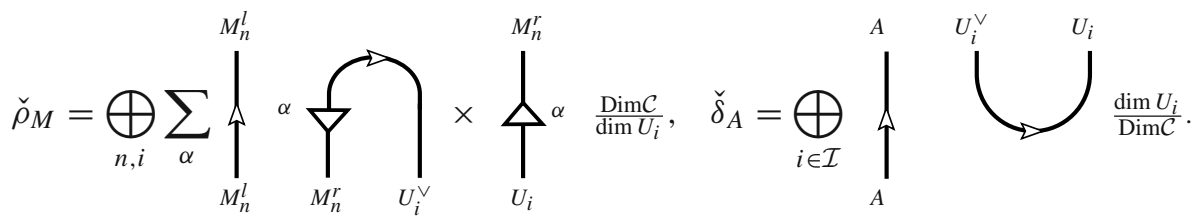

Note that

$$
\check{\rho}_{M} \circ \hat{\delta}_{M}=\operatorname{Dim} \mathcal{C} \cdot \operatorname{id}_{M} \text { and } \hat{\rho}_{A} \circ \check{\delta}_{A}=\operatorname{id}_{A} .
$$

Lemma 2.22. The functors $T$ and $R$ as maps on the sets of morphisms have left inverses, and thus are injective.

Proof. Let $f: A \rightarrow B$ be a morphism in $\mathcal{C}$. We define a map $Q_{R}: \operatorname{Hom}_{\mathcal{C}_{ \pm}^{2}}(R(A)$, $R(B)) \rightarrow \operatorname{Hom}_{\mathcal{C}}(A, B)$ by $f^{\prime} \mapsto \hat{\rho}_{B} \circ T\left(f^{\prime}\right) \circ \check{\delta}_{A}$. Then we have

$$
Q_{R} \circ R(f)=\hat{\rho}_{B} \circ T R(f) \circ \check{\delta}_{A}=\hat{\rho}_{B} \circ \check{\delta}_{B} \circ f=f,
$$


where we used naturality of $\check{\delta}$ and (2.50) in the second and third equalities, respectively. So $Q_{R}$ is a left inverse of $R$ on morphisms. Thus $R$ is injective on morphisms. Similarly, let $g: M \rightarrow N$ be a morphism in $\mathcal{C}_{ \pm}^{2}$. We define a map $Q_{T}: \operatorname{Hom}_{\mathcal{C}}(T(M), T(N)) \rightarrow$ $\operatorname{Hom}_{\mathcal{C}_{ \pm}^{2}}(M, N)$ by $g^{\prime} \mapsto(\operatorname{Dim} \mathcal{C})^{-1} \cdot \check{\rho}_{N} \circ R\left(g^{\prime}\right) \circ \hat{\delta}_{M}$. Then we have

$$
Q_{T} \circ T(g)=(\operatorname{Dim} \mathcal{C})^{-1} \cdot \check{\rho}_{N} \circ R T(g) \circ \hat{\delta}_{M}=(\operatorname{Dim} \mathcal{C})^{-1} \cdot \check{\rho}_{N} \circ \hat{\delta}_{N} \circ g=g .
$$

So $Q_{T}$ is a left inverse of $T$ on morphisms. Thus $T$ is injective on morphisms.

Using (2.9) and (2.50), one can express the two inverse maps $\hat{\chi}^{-1}, \check{\chi}^{-1}$ as follows, for $f \in \operatorname{Hom}_{\mathcal{C}_{ \pm}^{2}}(M, R(A))$ and $g \in \operatorname{Hom}_{\mathcal{C}_{ \pm}^{2}}(R(A), M)$,

$$
\hat{\chi}^{-1}(f)=\hat{\rho} \circ T(f), \quad \check{\chi}^{-1}(g)=T(g) \circ \check{\delta} .
$$

By Proposition 2.5 and Lemma 2.7, $R$ is both a lax and colax tensor functor. In particular, $\phi_{0}^{R}: \mathbf{1} \times \mathbf{1} \rightarrow R(\mathbf{1})$ is given by

$$
\phi_{0}^{R}=\hat{\chi}\left(\psi_{0}^{T}\right)=R\left(\psi_{0}^{T}\right) \circ \hat{\delta}_{\mathbf{1} \times \mathbf{1}}=\mathrm{id}_{\mathbf{1} \times \mathbf{1}}
$$

and $\phi_{2}^{R}: R(A) \otimes R(B) \rightarrow R(A \otimes B)$ by $\phi_{2}^{R}=R\left(\hat{\rho}_{A} \otimes \hat{\rho}_{B}\right) \circ R\left(\psi_{2}^{T}\right) \circ \hat{\delta}$, which can be expressed graphically as

$$
\phi_{2}^{R}=\left.\bigoplus_{i, j, k \in \mathcal{I}} \sum_{\alpha}\right|_{U_{i}^{\vee}} ^{A}
$$

Similarly, $\psi_{0}^{R}: R(\mathbf{1}) \rightarrow \mathbf{1} \times \mathbf{1}$ is given by

$$
\psi_{0}^{R}=\check{\rho}_{\mathbf{1}} \circ R\left(\phi_{0}^{T}\right)=\operatorname{Dim} \mathcal{C} \operatorname{id}_{\mathbf{1} \times \mathbf{1}}
$$

and $\psi_{2}^{R}: R(A \otimes B) \rightarrow R(A) \otimes R(B)$ by $\psi_{2}^{R}=\check{\rho} \circ R\left(\phi_{2}^{T}\right) \circ R\left(\check{\delta}_{A} \otimes \check{\delta}_{B}\right)$, which in graphical notation reads

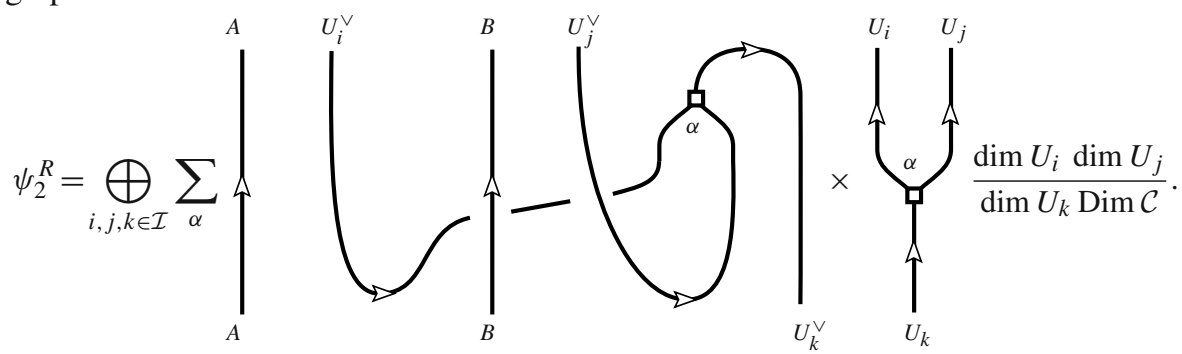

If $\mathcal{C}$ has more than one simple object, then $R$ does not take the tensor unit of $\mathcal{C}$ to the tensor unit of $\mathcal{C}_{ \pm}^{2}$ and so is clearly not a tensor functor. However, we will show that $R$ is still a Frobenius functor. This will imply that if $A$ is a Frobenius algebra in $\mathcal{C}$, then

$$
R(A)=\left(R(A), m_{R(A)}, \eta_{R(A)}, \Delta_{R(A)}, \varepsilon_{R(A)}\right)
$$


is a Frobenius algebra in $\mathcal{C}_{ \pm}^{2}$, where the structure morphisms were given in (2.17) and (2.18). In the case $A=\mathbf{1}$ it was proved in [Mü1, Prop.4.1] (see also [Fr, Lem.6.19] and [K1, Thm.5.2]) that (2.58) is a commutative simple symmetric normalised-special Frobenius algebra in $\mathcal{C}_{ \pm}^{2}$. In fact, given a Frobenius algebra $A$ in $\mathcal{C}$, it is straightforward to verify that the structure morphisms in (2.58) are precisely those of $(A \times \mathbf{1}) \otimes R(\mathbf{1})$, cf. Sect. 2.2.

Proposition 2.23. $\left(R, \phi_{2}^{R}, \phi_{0}^{R}, \psi_{2}^{R}, \psi_{0}^{R}\right)$ is a Frobenius functor.

Proof. Using the explicit graphical expression of $\phi_{2}^{R}, \phi_{0}^{R}, \psi_{2}^{R}, \psi_{0}^{R}$, it is easy to see that the commutativity of the diagrams (2.5) and (2.6) are equivalent to the statement that $R(\mathbf{1})$ with structure morphisms as in (2.58) is a Frobenius algebra in $\mathcal{C}_{ \pm}^{2}$. The latter statement is true by [Mü1, Prop.4.1].

From Lemma 2.20 and Proposition 2.23 we see that $T$ and $R$ take Frobenius algebras to Frobenius algebras. The following two propositions show how the properties of Frobenius algebras are transported.

Proposition 2.24. Let A be a Frobenius algebra in $\mathcal{C}_{ \pm}^{2}$. Then $T(A)$ is a Frobenius algebra in $\mathcal{C}$ and

(i) $A$ is symmetric iff $T(A)$ is symmetric.

(ii) $A$ is (normalised-)special iff $T(A)$ is (normalised-)special.

Proof. For part (i) write $A$ as a direct sum $\oplus_{n=1}^{N} A_{n}^{l} \times A_{n}^{r}$. Then the maps $I_{T(A)}, I_{T(A)}^{\prime}$ : $T\left(A^{\vee}\right) \rightarrow T(A)^{\vee}$ defined in (2.20) are given by:

$$
I_{T(A)}=I_{T(A)}^{\prime}=\bigoplus_{n=1}^{N} c_{\left(A_{n}^{l}\right)^{\vee},\left(A_{n}^{r}\right)^{\vee}} .
$$

Therefore, by (2.21), $\Phi_{T(A)}=\Phi_{T(A)}^{\prime}$ is equivalent to $T\left(\Phi_{A}\right)=T\left(\Phi_{A}^{\prime}\right)$. Since by Lemma 2.22, $T$ is injective on morphisms, this proves part (i). Part (ii) can be checked in the same way, for example the condition $m_{T(A)} \circ \Delta_{T(A)}=\zeta \operatorname{id}_{T(A)}$ is easily checked to be equivalent to $T\left(m_{A} \circ \Delta_{A}\right)=\zeta T\left(\mathrm{id}_{A}\right)$.

Proposition 2.25. Let $A$ be a Frobenius algebra $A$ in $\mathcal{C}$. Then $R(A)$ is a Frobenius algebra in $\mathcal{C}_{ \pm}^{2}$ and

(i) $A$ is symmetric iff $R(A)$ is symmetric.

(ii) $A$ is (normalised-)special iff $R(A)$ is (normalised-)special.

Proof. Recall that the structure morphisms of the Frobenius algebra $R(A)$ are equal to those of $(A \times \mathbf{1}) \otimes R(\mathbf{1})$. Using this equality, part (i) and (ii) follow because $R(\mathbf{1})$ is symmetric and normalised-special. For example,

$$
m_{R(A)} \circ \Delta_{R(A)}=\left[\left(m_{A} \circ \Delta_{A}\right) \times \mathrm{id}_{\mathbf{1}}\right] \otimes \mathrm{id}_{R(\mathbf{1})}=R\left(m_{A} \circ \Delta_{A}\right),
$$

so that $m_{R(A)} \circ \Delta_{R(A)}=\zeta \operatorname{id}_{R(A)}$ is equivalent to $R\left(m_{A} \circ \Delta_{A}\right)=\zeta R\left(\mathrm{id}_{A}\right)$, which by Lemma 2.22 is equivalent to $m_{A} \circ \Delta_{A}=\zeta \mathrm{id}_{A}$.

The functor $R$ has one additional property not shared by $T$, namely $R$ takes absolutely simple algebras to absolutely simple algebras. We will see explicitly in Sect. 3.3 that this is not true for $T$. 
Lemma 2.26. For a $\mathcal{C}$-algebra $A$, the map

$$
R: \operatorname{Hom}_{A \mid A}(A, A) \rightarrow \operatorname{Hom}_{R(A) \mid R(A)}(R(A), R(A))
$$

given by $f \mapsto R(f)$ is well-defined and an isomorphism.

Proof. Since $R$ is a lax tensor functor, $R(A)$ is naturally a $R(A)$-bimodule. It is easy to see that $R$ in (2.61) is a well-defined map. $R(A)$ is also naturally a $R(\mathbf{1})$-bimodule, which can be identified with the induced $R(\mathbf{1})$-bimodule structure on $(A \times \mathbf{1}) \otimes R(\mathbf{1})$, where the left $R(\mathbf{1})$ action on $(A \times \mathbf{1}) \otimes R(\mathbf{1})$ is given by $\left(\mathrm{id}_{A \times \mathbf{1}} \otimes m_{R(\mathbf{1})}\right) \circ\left(c_{A \times 1, R(\mathbf{1})}^{-1} \otimes \mathrm{id}_{R(\mathbf{1})}\right)$. We have the following natural isomorphisms:

$$
\operatorname{Hom}_{R(\mathbf{1}) \mid R(\mathbf{1})}(R(A), R(A)) \stackrel{\cong}{\rightarrow} \operatorname{Hom}_{\mathcal{C}_{ \pm}^{2}}(A \times \mathbf{1}, R(A)) \stackrel{\hat{\chi}^{-1}}{\longrightarrow} \operatorname{Hom}_{\mathcal{C}}(A, A) .
$$

which, by (2.53), are given by, for $f \in \operatorname{Hom}_{R(\mathbf{1}) \mid R(\mathbf{1})}(R(A), R(A))$,

$$
f \mapsto f^{\prime}=f \circ\left(\operatorname{id}_{A \times \mathbf{1}} \otimes \eta_{R(\mathbf{1})}\right) \mapsto f^{\prime \prime}=\hat{\rho} \circ T\left(f^{\prime}\right),
$$

and its inverse is given by, for $g \in \operatorname{Hom}_{\mathcal{C}}(A, A)$,

$$
g \mapsto g^{\prime}=R(g) \circ \hat{\delta} \mapsto g^{\prime \prime}=\left(\operatorname{id}_{A \times \mathbf{1}} \otimes m_{R(\mathbf{1})}\right) \circ\left(g^{\prime} \otimes \operatorname{id}_{R(\mathbf{1})}\right),
$$

where $g^{\prime \prime}$ is indeed a $R(\mathbf{1})$-bimodule map due to the commutativity of $R(\mathbf{1})$. It is easy to check that $g^{\prime \prime}=R(g)$ in (2.64). Therefore $R$ gives an isomorphism from $\operatorname{Hom}_{\mathcal{C}}(A, A)$ to $\operatorname{Hom}_{R(\mathbf{1}) \mid R(\mathbf{1})}(R(A), R(A))$. Moreover, one verifies that $R(g)$ is an $R(A)$-bimodule map iff $g$ is an $A$-bimodule map. In other words, $R: g \mapsto R(g)$ gives an isomorphism $\operatorname{Hom}_{A \mid A}(A, A) \stackrel{\cong}{\rightarrow} \operatorname{Hom}_{R(A) \mid R(A)}(R(A), R(A))$.

Corollary 2.27. Let $A$ be a $\mathcal{C}$-algebra.

(i) A is absolutely simple iff $R(A)$ is absolutely simple.

(ii) Let $A$ be in addition Frobenius. Then $A$ is simple and special iff $R(A)$ is simple and special.

Proof. Part (i) immediately follows from Lemma 2.26. The statement of part (ii) without the qualifier 'simple' is proved in Proposition 2.25. But, as in the proof of (iii) $\Rightarrow$ (i) in Lemma 2.11, a special Frobenius algebra in a semi-simple category has a semi-simple category of bimodules, and for a semi-simple $\mathbb{C}$-linear category, simple and absolutely simple are equivalent. Part (ii) then follows from part (i).

The following lemma will be needed in Sect. 3.2 below to discuss the properties of Cardy algebras.

Lemma 2.28. Let $A$ be a Frobenius algebra in $\mathcal{C}$. Then $\left(\check{\delta}_{A}\right)^{*}=\hat{\rho}_{A}$.

Proof. Recall from (2.50) that $\check{\delta}_{A}$ is a morphism $A \rightarrow T R(A)$. Since $T$ and $R$ are both Frobenius functors, $T R(A)$ is a Frobenius algebra in $\mathcal{C}$. Substituting the definitions, after a short calculation one finds 


$$
\varepsilon_{T R(A)} \circ m_{T R(A)}=\operatorname{Dim}(\mathcal{C}) \bigoplus_{i \in \mathcal{I}}
$$

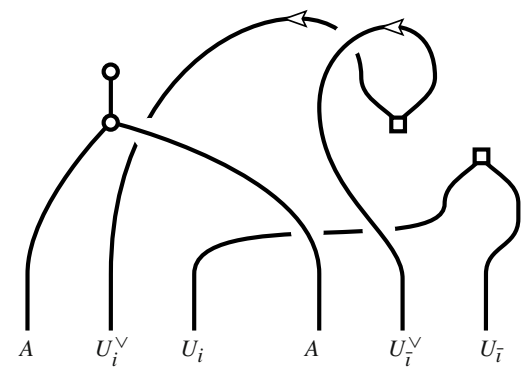

Substituting this in the definition of $\left(\check{\delta}_{A}\right)^{*}$ gives, again after a short calculation, the morphism $\hat{\rho}_{A}$. At an intermediate step one uses that the part of the morphism $\left(\check{\delta}_{A}\right)^{*}$, which is made up of $U_{i}$ and $U_{\bar{l}}$ ribbons, their duals, and the basis morphisms $\lambda_{(i, \bar{l}) 0}$ and $\Upsilon^{(i, \bar{l}) 0}$, can be replaced by $\frac{1}{\operatorname{dim} U_{i}} \cdot d_{U_{i}}$.

\section{Cardy Algebras}

In this section we start by investigating the properties of Frobenius algebras which satisfy the so-called modular invariance condition. We then give two definitions of a Cardy algebra and prove their equivalence. Finally, in Sect. 3.3, we study the properties of these algebras and state our main results.

We fix a modular tensor category $\mathcal{C}$. Recall that $\mathcal{C}_{ \pm}^{2}$ is an abbreviation for $\mathcal{C}_{+} \otimes \mathcal{C}_{-}$.

3.1. Modular invariance. In $\mathcal{C}_{ \pm}^{2}$, we define the object $K$ and the morphism $\omega: K \rightarrow K$ as

$$
K=\bigoplus_{i, j \in \mathcal{I}} U_{i} \times U_{j}, \quad \omega=\sum_{i, j \in \mathcal{I}} \frac{\operatorname{dim} U_{i} \operatorname{dim} U_{j}}{\operatorname{Dim} \mathcal{C}} \mathrm{id}_{U_{i} \times U_{j}}
$$

They have the property (see e.g. [BK, Cor. 3.1.11])

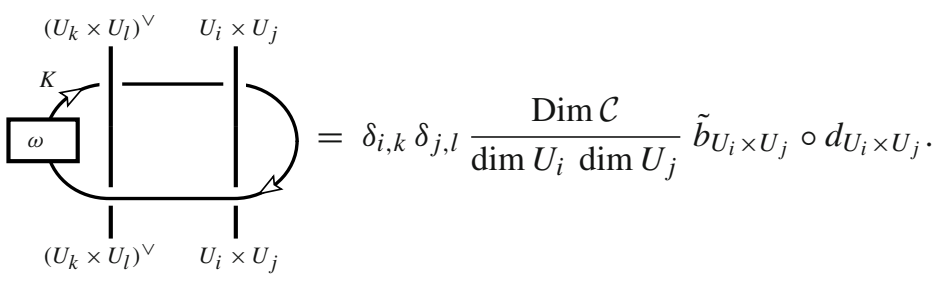




\section{Definition 3.1.}

(i) Let $A, B$ be objects of $\mathcal{C}_{ \pm}^{2}$. A morphism $f: A \otimes B \rightarrow B$ is called $\mathbf{S}$-invariant iff

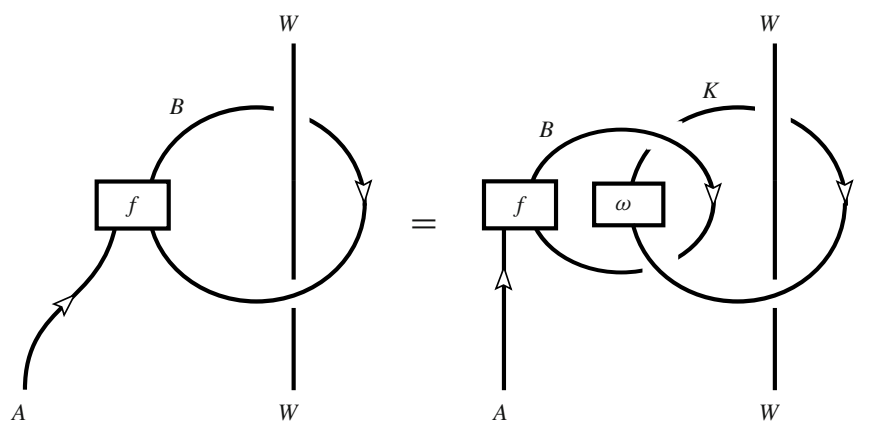

holds for all for $W \in \mathcal{C}_{ \pm}^{2}$.

(ii) $A \mathcal{C}_{ \pm}^{2}$-algebra $\left(A_{\mathrm{cl}}, m_{\mathrm{cl}}, \eta_{\mathrm{cl}}\right)$ is called modular invariant iff $\theta_{A_{\mathrm{cl}}}=\mathrm{id}_{A_{\mathrm{cl}}}$ and $m_{\mathrm{cl}}$ is $S$-invariant.

Lemma 3.2. The morphism $f: A \otimes B \rightarrow B$ is $S$-invariant if and only if

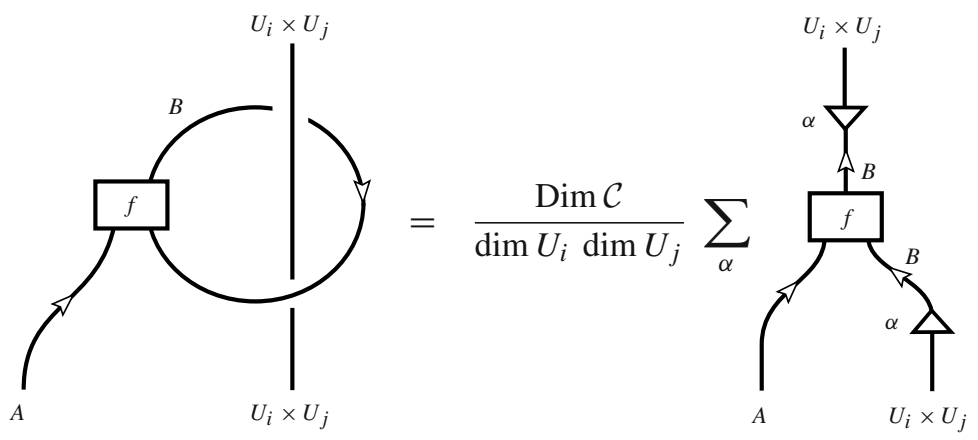

holds for all $i, j \in \mathcal{I}$.

Proof. Condition (3.3) holds for all $W$ iff it holds for all $W=U_{i} \times U_{j}, i, j \in \mathcal{I}$, so it is enough to show that the right-hand side of (3.3) with $W=U_{i} \times U_{j}$ is equal to the right-hand side of (3.4). Recall the notation for basis morphisms in (2.36). Starting from (3.3), write

$$
\operatorname{id}_{B^{\vee}} \otimes \operatorname{id}_{U_{i} \times U_{j}}=\left(\sum_{k, l, \alpha}\left(b_{B}^{(k \times l ; \alpha)}\right)^{\vee} \circ\left(b_{(k \times l ; \alpha)}^{B}\right)^{\vee}\right) \otimes \operatorname{id}_{U_{i} \times U_{j}},
$$

and then apply (3.2). The graphical representation of the resulting morphism can be deformed to give (3.4).

Remark 3.3. As shown in [K3, Sect. 6.1], the modular invariance condition of a $\mathcal{C}_{ \pm}^{2}$-algebra exactly coincides with the modular invariance condition for torus 1-point correlation functions of a genus-0,1 closed CFT. In particular, the condition $\theta_{A_{\mathrm{cl}}}=\mathrm{id}_{A_{\mathrm{cl}}}$ is equivalent to invariance under the modular transformation $T: \tau \mapsto \tau+1$, and the condition (3.4) with $f=m_{\mathrm{cl}}$ is equivalent to invariance under $S: \tau \mapsto-\frac{1}{\tau}$. Combining the modular invariance condition with the genus zero properties of a genus- 0,1 closed CFT results in a modular invariant commutative symmetric Frobenius algebra in $\mathcal{C}_{ \pm}^{2}$. 
Let $A_{\mathrm{cl}}$ be a modular invariant $\mathcal{C}_{ \pm}^{2}$-algebra. Evaluating (3.4) for $f=m_{\mathrm{cl}}$, composing it with $\eta_{\mathrm{cl}} \otimes \mathrm{id}_{U_{i} \times U_{j}}$ and taking the trace implies the following identity:

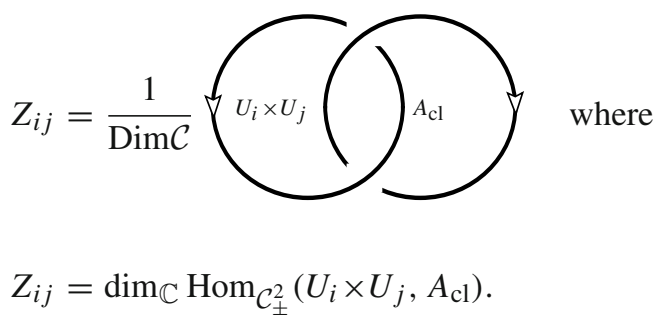

Decomposing $A_{\mathrm{cl}}$ into simple objects, this gives

$$
Z_{i j}=\sum_{k, l \in \mathcal{I}} S_{i k} Z_{k l} S_{l j}^{-1} \quad \text { where } S_{i j}=s_{i, j} / \sqrt{\operatorname{DimC}}
$$

which in CFT terms is of course nothing but the invariance of the torus partition function under the modular S-transformation.

The following theorem gives a simple criterion for modular invariance.

Theorem 3.4. Let $A_{\mathrm{cl}}$ be a haploid commutative symmetric Frobenius algebra in $\mathcal{C}_{ \pm}^{2}$.

(i) If $A_{\mathrm{cl}}$ is modular invariant, then $\operatorname{dim} A_{\mathrm{cl}}=\operatorname{Dim} \mathcal{C}$.

(ii) If $\operatorname{dim} A_{\mathrm{cl}}=\operatorname{Dim} \mathcal{C}$, then $A_{\mathrm{cl}}$ is special and modular invariant.

Proof. Part (i): Since $A_{\mathrm{cl}}$ is haploid, for $i=j=0$, Equation (3.6) reduces to $1=$ $\operatorname{dim} A_{\mathrm{cl}} / \operatorname{Dim} \mathcal{C}$.

Part (ii): By the same reasoning as in the proof of (iii) $\Rightarrow$ (i) in Lemma 2.11 one shows that $A_{\mathrm{cl}}$ is special. Thus $m_{\mathrm{cl}} \circ \Delta_{\mathrm{cl}}=\zeta_{\mathrm{cl}} \mathrm{id}_{A_{\mathrm{cl}}}$ for some $\zeta_{\mathrm{cl}} \neq 0$.

By [KO, Thm.4.5], the category $\left(\mathcal{C}_{ \pm}^{2}\right)_{A_{\mathrm{cl}}}^{\text {loc }}$ of local $A_{\mathrm{cl}}$-modules is again a modular tensor category and $\operatorname{Dim}\left(\mathcal{C}_{ \pm}^{2}\right)_{A_{\mathrm{cl}}}^{\text {loc }}=\left(\operatorname{Dim} \mathcal{C} / \operatorname{dim} A_{\mathrm{cl}}\right)^{2}$ (see [Fr, Prop. $3.21 \&$ Rem.3.23] for the same statement in the notation used here). Thus by assumption we have $\operatorname{Dim}\left(\mathcal{C}_{ \pm}^{2}\right)_{A_{\mathrm{cl}}}^{l o c}=1$. It then follows from [ENO1, Thm. 2.3] that up to isomorphism, $\left(\mathcal{C}_{ \pm}^{2}\right)_{A_{\mathrm{cl}}}^{\text {loc }}$ has a unique simple object (namely the tensor unit). In other words, every simple local $A_{\mathrm{cl}}$-module is isomorphic to $A_{\mathrm{cl}}$ (seen as a left-module over itself).

We have the following isomorphisms between morphism spaces [FS, Prop. $4.7 \& 4.11$ ],

$$
\begin{aligned}
& \operatorname{Hom}_{A_{\mathrm{cl}}}\left(A_{\mathrm{cl}} \otimes\left(U_{i} \times U_{j}\right), A_{\mathrm{cl}}\right) \cong \operatorname{Hom}_{\mathcal{C}_{ \pm}^{2}}\left(U_{i} \times U_{j}, A_{\mathrm{cl}}\right), \\
& \operatorname{Hom}_{A_{\mathrm{cl}}}\left(A_{\mathrm{cl}}, A_{\mathrm{cl}} \otimes\left(U_{i} \times U_{j}\right)\right) \cong \operatorname{Hom}_{\mathcal{C}_{ \pm}^{2}}\left(A_{\mathrm{cl}}, U_{i} \times U_{j}\right) .
\end{aligned}
$$

Using these to transport the bases (2.36) from the right to the left, we obtain bases $\left\{b_{(i j)}^{\alpha}\right\}_{\alpha}$ of $\operatorname{Hom}_{A_{\mathrm{cl}}}\left(A_{\mathrm{cl}} \otimes\left(U_{i} \times U_{j}\right), A_{\mathrm{cl}}\right)$ and $\left\{b_{\beta}^{(i j)}\right\}_{\beta}$ of $\operatorname{Hom}_{A_{\mathrm{cl}}}\left(A_{\mathrm{cl}}, A_{\mathrm{cl}} \otimes\left(U_{i} \times U_{j}\right)\right)$. These can be expressed graphically as 


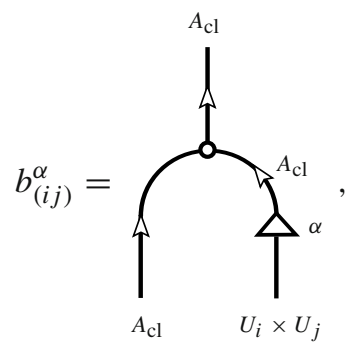

$$
b_{\beta}^{(i j)}=\frac{\operatorname{DimC}}{\operatorname{dim} U_{i} \operatorname{dim} U_{j}} \frac{1}{\zeta_{\mathrm{cl}}}
$$

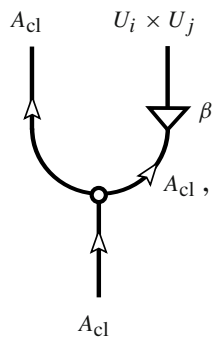

where the nonzero factor in $b_{\beta}^{(i j)}$ is included for convenience. Notice that $b_{(i j)}^{\alpha} \circ b_{\beta}^{(i j)}$ is a left $A_{\mathrm{cl}}$-module map. Since $A_{\mathrm{cl}}$ is simple as a left module over itself, we have

$$
b_{(i j)}^{\alpha} \circ b_{\beta}^{(i j)}=\lambda_{\alpha \beta} \mathrm{id}_{A_{\mathrm{cl}}}
$$

for some $\lambda_{\alpha \beta} \in \mathbb{C}$. By computing $\operatorname{tr}\left(b_{(i j)}^{\alpha} \circ b_{\beta}^{(i j)}\right)$, it is easy to verify that $\lambda_{\alpha \beta}=\delta_{\alpha \beta}$.

We will now prove the following identity:
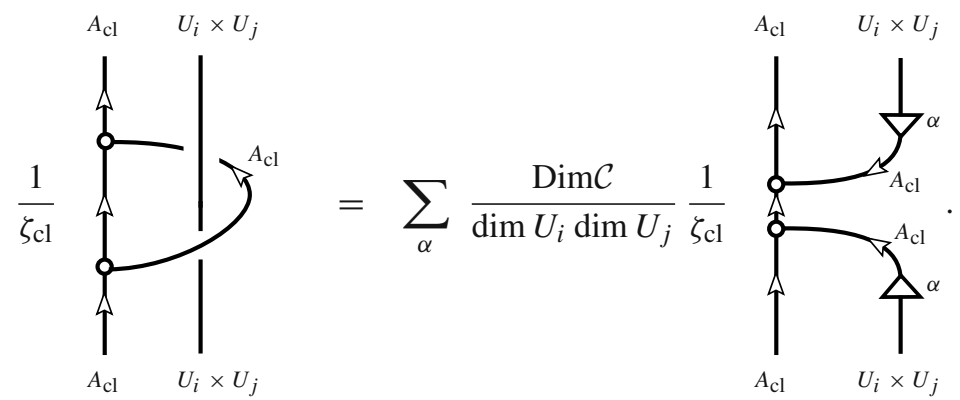

One checks that the left-hand side of this equation is an idempotent, which we denote by $\zeta_{\mathrm{cl}}^{-1} P_{A_{\mathrm{cl}}}^{l}\left(U_{i} \times U_{j}\right)$, cf. [Fr, Sect. 3.1]. By [Fr, Prop. 4.1] the image $\operatorname{Im}\left(\zeta_{\mathrm{cl}}^{-1} P_{A_{\mathrm{cl}}}^{l}\left(U_{i} \times U_{j}\right)\right)$ is a local $A_{\mathrm{cl}}$-module, and hence isomorphic to $A_{\mathrm{cl}}^{\oplus N}$ for some $N \in \mathbb{Z}_{\geq 0}$.

All left-module morphisms from $A_{\mathrm{cl}}$ to $A_{\mathrm{cl}} \otimes\left(U_{i} \times U_{j}\right)$ are linear combinations of the $b_{\beta}^{(i j)}$. Furthermore one verifies that $\zeta_{\mathrm{cl}}^{-1} P_{A_{\mathrm{cl}}}^{l}\left(U_{i} \times U_{j}\right) \circ b_{\beta}^{(i j)}=b_{\beta}^{(i j)}$. Therefore, the $b_{\beta}^{(i j)}$ describe precisely the image of the idempotent, i.e. $\zeta_{\mathrm{cl}}^{-1} P_{A_{\mathrm{cl}}}^{l}\left(U_{i} \times U_{j}\right)=\sum_{\alpha} b_{\alpha}^{(i j)} \circ b_{(i j)}^{\alpha}$, which is nothing but (3.10). Composing (3.10) with $\zeta_{\mathrm{cl}} \cdot \varepsilon_{\mathrm{cl}} \otimes \mathrm{id}_{U_{i} \times U_{j}}$ from the left (i.e. from the top) produces (3.4).

In addition, since $A_{\mathrm{cl}}$ is commutative symmetric Frobenius it satisfies $\theta_{A_{\mathrm{cl}}}=\mathrm{id}_{A_{\mathrm{cl}}}$ [Fr, Prop. 2.25]. Altogether, this shows that $A_{\mathrm{cl}}$ is modular invariant.

Remark 3.5. As we were writing this paper, we heard that the results in Theorem 3.4 were obtained independently by Kitaev and Müger [Ki].

Remark 3.6. Setting $i=j=0$ in (3.6) gives the identity $\operatorname{dim} A_{\mathrm{cl}}=Z_{00} \operatorname{Dim} \mathcal{C}[\mathrm{KR}$, Prop. 2.3]. Combining this with Theorem 3.4(ii) one may wonder if a general modular invariant commutative symmetric Frobenius algebra $A_{\mathrm{cl}}$ in $\mathcal{C}_{ \pm}^{2}$ is isomorphic to a direct sum of simple such algebras. However, this is not so. For example, one can take the commutative symmetric Frobenius algebra $A_{\mathrm{cl}}=\mathbb{C}[x] / x^{2}$ in the category of vector spaces equipped with the non-degenerate trace $\varepsilon(a x+b)=a$. In this case the modular invariance condition holds automatically, but $A_{\mathrm{cl}}$ is clearly not a direct sum of two algebras. 
For a general modular tensor category $\mathcal{C}$, the algebra $\mathbb{C}[x] / x^{2} \otimes R(\mathbf{1})$, understood as an algebra in $\mathcal{C}_{ \pm}^{2}$ via the braided monoidal isomorphism $\mathcal{V} e c t_{f}(\mathbb{C}) \otimes \mathcal{C}_{ \pm}^{2} \rightarrow \mathcal{C}_{ \pm}^{2}$, provides another counter-example.

3.2. Two definitions. Define a morphism $P_{A}^{l}: A \rightarrow A$ for a Frobenius algebra $A$ in $\mathcal{C}$ or $\mathcal{C}_{ \pm}^{2}$ as follows [Fr, Sect. 2.4],

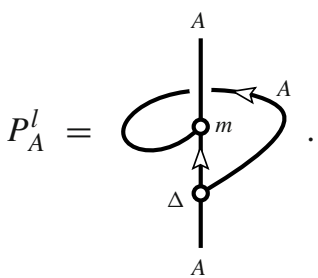

If $A$ is also commutative and obeys $m_{A} \circ \Delta_{A}=\zeta_{A} \mathrm{id}_{A}$, we have $P_{A}^{l}=\zeta_{A} \mathrm{id}_{A}$. In particular, this holds if $A$ is commutative and special. Using the fact that the Frobenius algebra $R(\mathbf{1})$ is commutative and normalised-special, one can check that $P_{R(A)}^{l}: R(A) \rightarrow R(A)$ takes the following form:

$$
P_{R(A)}^{l}=\bigoplus_{i \in \mathcal{I}}
$$

With these ingredients, we can now give the first definition of a Cardy $\mathcal{C} \mid \mathcal{C}_{ \pm}^{2}$-algebra, which was introduced in [K3, Def. 5.14], cf. Remark 3.15 below.

Definition 3.7 (Cardy $\mathcal{C} \mid \mathcal{C}_{ \pm}^{2}$-algebra I). A Cardy $\mathcal{C} \mid \mathcal{C}_{ \pm}^{2}$-algebra is a triple $\left(A_{\mathrm{op}} \mid A_{\mathrm{cl}}, \iota_{\mathrm{cl}-\mathrm{op}}\right)$, where $\left(A_{\mathrm{cl}}, m_{\mathrm{cl}}, \eta_{\mathrm{cl}}, \Delta_{\mathrm{cl}}, \varepsilon_{\mathrm{cl}}\right)$ is a modular invariant commutative symmetric Frobenius $\mathcal{C}_{ \pm}^{2}$-algebra, $\left(A_{\mathrm{op}}, m_{\mathrm{op}}, \eta_{\mathrm{op}}, \Delta_{\mathrm{op}}, \varepsilon_{\mathrm{op}}\right)$ is a symmetric Frobenius $\mathcal{C}$-algebra, and $\iota_{\mathrm{cl}-\mathrm{op}}: A_{\mathrm{cl}} \rightarrow R\left(A_{\mathrm{op}}\right)$ an algebra homomorphism, such that the following conditions are satisfied:

(i) Centre condition:
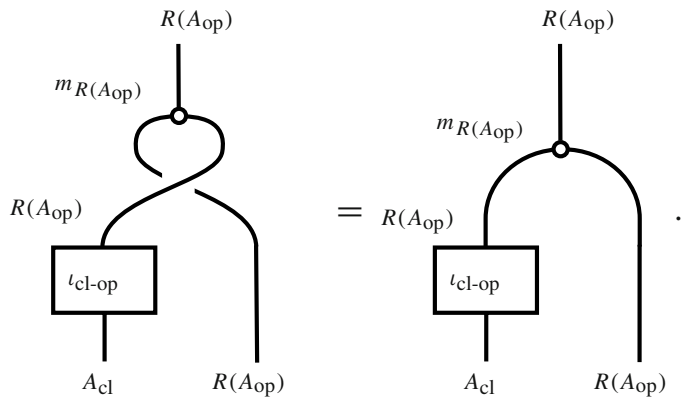
(ii) Cardy condition:

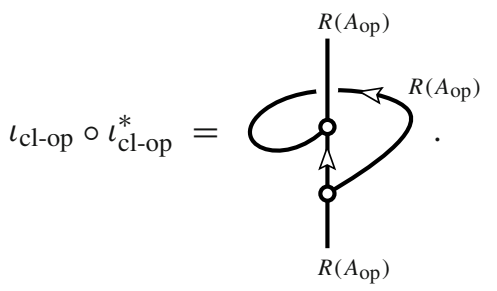

Remark 3.8.

(i) The name "Cardy $\mathcal{C} \mid \mathcal{C}_{ \pm}^{2}$-algebra" in Definition 3.7 was chosen because many of the important ingredients were first studied by Cardy: the modular invariance of the closed theory [C1], the consistency of the annulus amplitude [C2], and the bulk-boundary OPE [CL]. On the other hand, the boundary-boundary OPE and the OPE analogue of the centre condition were first considered in [Lw].

(ii) One can easily see that in the special case that $\mathcal{C}$ is the category $\mathcal{V} e c t_{f}(\mathbb{C})$ of finitedimensional $\mathbb{C}$-vector spaces, a Cardy $\mathcal{C} \mid \mathcal{C}_{ \pm}^{2}$-algebra gives exactly the algebraic formulation of two-dimensional open-closed topological field theory over $\mathbb{C}$ (cf. Remark 6.14 in [K3]), see [Lz, Sect.4.8], [Mo, Thm.1.1], [AN, Thm.4.5], [LP, Cor. 4.3], [MS, Sect.2.2]. When passing to a general modular tensor category $\mathcal{C}$ there are two important differences to the two-dimensional topological field theory. Firstly, the algebras $A_{\mathrm{cl}}$ and $A_{\mathrm{op}}$ now live in different categories, which in particular affects the formulation of the centre condition and the Cardy condition. Secondly, the modular invariance condition has to be imposed on $A_{\mathrm{cl}}$. In the case $\mathcal{C}=$ Vect $_{f}(\mathbb{C})$, modular invariance holds automatically.

Definition 3.9. A homomorphism of Cardy $\mathcal{C} \mid \mathcal{C}_{ \pm}^{2}$-algebras $\left(A_{\mathrm{op}}^{(1)} \mid A_{\mathrm{cl}}^{(1)}, \iota_{\mathrm{cl}-\mathrm{op}}^{(1)}\right) \rightarrow\left(A_{\mathrm{op}}^{(2)}\right.$ $\left.\mid A_{\mathrm{cl}}^{(2)}, \iota_{\mathrm{cl}-\mathrm{op}}^{(2)}\right)$ is a pair $\left(f_{\mathrm{op}}, f_{\mathrm{cl}}\right)$ of Frobenius algebra homomorphisms $f_{\mathrm{op}}: A_{\mathrm{op}}^{(1)} \rightarrow A_{\mathrm{op}}^{(2)}$ and $f_{\mathrm{cl}}: A_{\mathrm{cl}}^{(1)} \rightarrow A_{\mathrm{cl}}^{(2)}$ such that the diagram

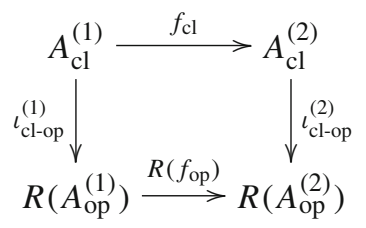

commutes.

Remark 3.10. Since a homomorphism of Frobenius algebras is invertible (cf. Lemma 2.18 (iv)), a homomorphism of Cardy algebras is always an isomorphism.

For a homomorphism $\left(f_{\mathrm{op}}, f_{\mathrm{cl}}\right)$ of Cardy $\mathcal{C} \mid \mathcal{C}_{ \pm}^{2}$-algebras, using the commutativity of (3.15) and the fact that $f_{\mathrm{cl}}$ and $f_{\mathrm{op}}$ are both algebra and coalgebra homomorphisms, it is easy to show that (3.15) commutes iff

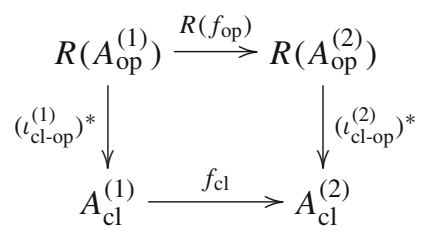


commutes.

Let $\left(A_{\text {op }} \mid A_{\mathrm{cl}}, \iota_{\mathrm{cl}-\mathrm{op}}\right)$ be a Cardy $\mathcal{C} \mid \mathcal{C}_{ \pm}^{2}$-algebra. Define the morphism

$$
\tilde{\iota}_{\mathrm{cl}-\mathrm{op}}=\hat{\chi}^{-1}\left(\iota_{\mathrm{cl}-\mathrm{op}}\right): T\left(A_{\mathrm{cl}}\right) \longrightarrow A_{\mathrm{op}} .
$$

Decompose $A_{\mathrm{cl}}$ as $A_{\mathrm{cl}}=\bigoplus_{n=1}^{N} C_{n}^{l} \times C_{n}^{r}$ such that $C_{1}^{l} \times C_{1}^{r}=\eta_{\mathrm{cl}}(\mathbf{1} \times \mathbf{1})$. We use $\iota_{\mathrm{cl}-\mathrm{op}}^{(n)}$ to denote the restriction of $\iota_{\mathrm{cl} \text {-op }}$ to $C_{n}^{l} \times C_{n}^{r}$ and $\tilde{\iota}_{\mathrm{cl}-\mathrm{op}}^{(n)}$ to denote the restriction of $\tilde{\iota}_{\mathrm{cl}-\mathrm{op}}$ to $C_{n}^{l} \otimes C_{n}^{r}$. We introduce the following graphical notation:

$$
\tilde{\iota}_{\mathrm{cl}-\mathrm{op}}^{(n)}=\overbrace{C_{n}^{l}}^{A_{\mathrm{op}}} .
$$

By $(2.43), \iota_{\mathrm{cl}-\text { op }}$ can be expressed in terms of $\tilde{\iota}_{\mathrm{cl}-\mathrm{op}}$ as follows:

$$
\iota_{\mathrm{cl}-\mathrm{op}}=\bigoplus_{n=1}^{N} \sum_{i \in \mathcal{I}} \overbrace{\alpha}^{C_{n}^{r}} \times \underbrace{\alpha}_{C_{n}^{l}} .
$$

Lemma 3.11. The centre condition (3.13) is equivalent to the following condition in $\mathcal{C}$ :

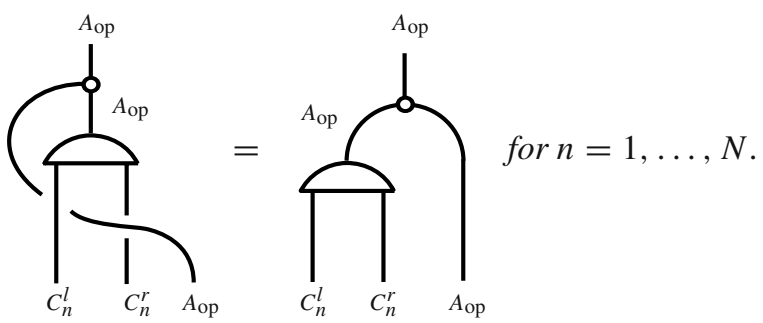

Proof. First, insert (3.19) and the definition (2.17) of $m_{R\left(A_{\mathrm{op}}\right)}$ into (3.13). Then apply the commutativity of $R(\mathbf{1})$ to the left hand side of (3.13). The equivalence between (3.13) and (3.20) follows immediately.

Remark 3.12. The centre condition (3.20) is very natural from the open-closed conformal field theory point of view. Correlators on the upper half plane are expressed in terms of conformal blocks on the full complex plane. The objects $C_{n}^{l}$ and $C_{n}^{r}$ are associated to the field insertion at a point $z$ in the upper half plane and at the complex conjugate point $\bar{z}$ in the lower half plane, respectively. The object $A_{\text {op }}$ corresponds to a field inserted at a point $r$ on the real axis. The centre condition (3.20) simply says that the correlation functions in the disjoint domains $|z|>r>0$ and $r>|z|>0$ are analytic continuations of each other, see [K2, Prop. 1.18]. 
Recall that we define $\tilde{\iota}_{\text {cl-op }}^{*}: A_{\mathrm{op}} \rightarrow T\left(A_{\mathrm{cl}}\right)$ as in (2.30). We introduce the graphical notation

$$
\tilde{\iota}_{\mathrm{cl}-\mathrm{op}}^{*}=\bigoplus_{n=1}^{N} \overbrace{A_{\mathrm{op}}}^{C_{n}^{l}}=\bigoplus_{A_{\mathrm{op}}}^{C_{n}^{r}}
$$

where the second equality follows from (2.15), (2.21) and (2.59).

Lemma 3.13. The Cardy condition (3.14) is equivalent to the following identity in $\mathcal{C}$ :

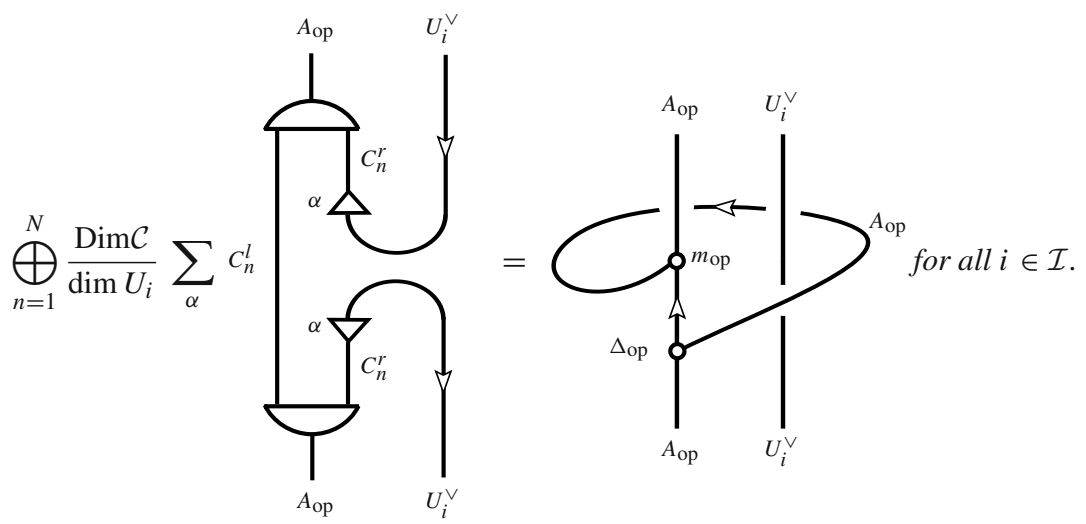

Proof. By (2.44), (3.12) and (3.19), it is easy to see that (3.22) is equivalent to the following identity:

$$
\iota_{\mathrm{cl}-\mathrm{op}} \circ \check{\chi}\left(\tilde{\iota}_{\mathrm{cl}-\mathrm{op}}^{*}\right)=P_{R\left(A_{\mathrm{op}}\right)}^{l} .
$$

Therefore, it is enough to show that

$$
\check{\chi}\left(\tilde{\iota}_{\mathrm{cl}-\mathrm{op}}^{*}\right)=\iota_{\mathrm{cl}-\mathrm{op}}^{*}
$$

We have

$$
\check{\chi}^{-1}\left(\iota_{\mathrm{cl}-\mathrm{op}}^{*}\right) \stackrel{(1)}{=} T\left(\iota_{\mathrm{cl}-\mathrm{op}}^{*}\right) \circ \check{\delta} \stackrel{(2)}{=} T\left(\iota_{\mathrm{cl}-\mathrm{op}}\right)^{*} \circ \check{\delta}^{* *} \stackrel{(3)}{=}\left(\hat{\rho} \circ T\left(\iota_{\mathrm{cl}-\mathrm{op}}\right)\right)^{*} \stackrel{(4)}{=}\left(\tilde{\iota}_{\mathrm{cl}-\mathrm{op}}\right)^{*}
$$

In step (1) we use the expression (2.53) for $\check{\chi}^{-1}$, step (2) follows from Lemma 2.18 (v) and Lemma 2.19. Step (3) is Lemma 2.18(i) and Lemma 2.28, and finally step (4) amounts to substituting (2.53) and (3.17). Acting with $\check{\chi}$ on both sides of the above equality produces (3.24).

Combining Lemmas 3.11 and 3.13, and Proposition 2.16, we obtain the following equivalent definition of Cardy $\mathcal{C} \mid \mathcal{C}_{ \pm}^{2}$-algebra (recall the graphical notation (3.18) for $\tilde{\iota}_{\mathrm{cl}-\mathrm{op}}$ and (3.21) for $\tilde{\iota}_{\mathrm{cl}-\mathrm{op}}^{*}$. 
Definition 3.14 (Cardy $\mathcal{C} \mid \mathcal{C}_{ \pm}^{2}$-algebra II). A Cardy $\mathcal{C}$-algebra is a triple $\left(A_{\mathrm{op}} \mid A_{\mathrm{cl}}, \tilde{\iota}_{\mathrm{cl}-\mathrm{op}}\right)$, where $A_{\mathrm{cl}}$ is a commutative symmetric Frobenius $\mathcal{C}_{ \pm}^{2}$-algebra satisfying property (3.4) with $f=m_{\mathrm{cl}}, A_{\mathrm{op}}$ is a symmetric Frobenius $\mathcal{C}$-algebra, and $\tilde{\iota}_{\mathrm{cl}-\mathrm{op}}: T\left(A_{\mathrm{cl}}\right) \rightarrow A_{\mathrm{op}}$ is an algebra homomorphism satisfying the conditions (3.20) and (3.22).

Remark 3.15. Up to a choice of normalisation, Definition 3.14 is the same as the original one in [K3, Def.6.13]. The difference between the two definitions is the factor $\operatorname{Dim} \mathcal{C} / \operatorname{dim} U_{i}$ on the left hand side of (3.22), which in [K3, Def.6.13] is given by $\sqrt{\operatorname{Dim} \mathcal{C}} / \operatorname{dim} U_{i}$. The two definitions are related by rescaling the coproduct $\Delta_{\mathrm{cl}}$ and counit $\varepsilon_{\mathrm{cl}}$ of $A_{\mathrm{cl}}$ by $1 / \sqrt{\operatorname{Dim\mathcal {C}}}$ and $\sqrt{\operatorname{Dim\mathcal {C}}}$, respectively. We chose the convention in (3.22) to remove all dimension factors from the expression (3.14) for the Cardy condition.

3.3. Uniqueness and existence theorems. In this subsection we investigate the structure of Cardy algebras. We start with the following proposition, which, when combined with the results of part II, provides an alternative proof of [Fj, Prop. 4.22].

Proposition 3.16. Let $\left(A_{\mathrm{op}} \mid A_{\mathrm{cl}}, \iota_{\mathrm{cl}-\mathrm{op}}\right)$ be a Cardy $\mathcal{C} \mid \mathcal{C}_{ \pm}^{2}$-algebra. If $A_{\mathrm{cl}}$ is simple and $\operatorname{dim} A_{\mathrm{op}} \neq 0$, then $A_{\mathrm{op}}$ is simple and special.

Proof. By Remark 3.6, we have $\operatorname{dim} A_{\mathrm{cl}}=Z_{00} \operatorname{Dim} \mathcal{C} \neq 0$, and by Lemma 2.11, $A_{\mathrm{cl}}$ is therefore haploid. Restricting the Cardy condition (3.22) to the case $U_{i}=1$ and composing both sides with $\varepsilon_{\text {op }}$ from the left, we see that $\varepsilon_{\text {op }}$ kills all terms associated to $U_{j} \times \mathbf{1} \in A_{\mathrm{cl}}$ in the sum except for a single $\mathbf{1} \times \mathbf{1}$ term. Thus we obtain the following identity:

$$
\beta \varepsilon_{\mathrm{op}}=\tilde{d}_{A_{\mathrm{op}}} \circ\left(m_{\mathrm{op}} \otimes \mathrm{id}_{A_{\mathrm{op}}}\right) \circ\left(\mathrm{id}_{A_{\mathrm{op}}} \otimes b_{A_{\mathrm{op}}}\right),
$$

where $\beta \in \mathbb{C}$. Composing with $\eta_{\mathrm{op}}$ from the right in turn implies that $\beta \varepsilon_{\mathrm{op}} \circ \eta_{\mathrm{op}}=$ $\operatorname{dim} A_{\text {op }}$, which is nonzero by assumption. Thus also $\beta \neq 0$ and $\varepsilon_{\text {op }}$ is a nonzero multiple of the morphism on the right hand side of (3.26). By [FRS, Lem.3.11], $A_{\text {op }}$ is special.

Since $A_{\text {op }}$ is a special Frobenius algebra, $A_{\text {op }}$ is semi-simple as an $A_{\text {op-bimodule }}$ (apply [FS, Prop. 5.24] to $A_{\text {op }}$ tensored with its opposite algebra). Suppose $A_{\text {op }}$ is not simple, so that we can write $A_{\mathrm{op}}=A_{\mathrm{op}}^{(1)} \oplus A_{\mathrm{op}}^{(2)}$ for nonzero $A_{\mathrm{op}}$-bimodules $A_{\mathrm{op}}^{(1)}$ and $A_{\mathrm{op}}^{(2)}$. We denote the canonical embeddings and projections associated to this decomposition as $\iota_{1,2}$ and $\pi_{1,2}$. We have the identities

$$
m_{\mathrm{op}} \circ\left(\iota_{1} \otimes \iota_{2}\right)=0, \quad \varepsilon_{\mathrm{op}} \circ \eta_{\mathrm{op}}=\sum_{i=1}^{2} \varepsilon_{\mathrm{op}} \circ \iota_{i} \circ \pi_{i} \circ \eta_{\mathrm{op}} .
$$

The first identity follows since $\pi_{1} \circ m_{\mathrm{op}} \circ\left(\iota_{1} \otimes \iota_{2}\right)=0$ (as $m_{\mathrm{op}}$ gives the left action of $A_{\mathrm{op}}$ on $A_{\mathrm{op}}$ and hence it preserves $\left.A_{\mathrm{op}}^{(2)}\right)$, and similarly $\pi_{2} \circ m_{\mathrm{op}} \circ\left(\iota_{1} \otimes \iota_{2}\right)=0$. The second identity is just the completeness of $\iota_{1,2}, \pi_{1,2}$.

Since $\varepsilon_{\mathrm{op}} \circ \eta_{\mathrm{op}} \neq 0$, without losing generality we can assume $\varepsilon_{\mathrm{op}} \circ \iota_{1} \circ \pi_{1} \circ \eta_{\mathrm{op}} \neq 0$. Using that $\pi_{2}$ is a bimodule map we compute

$$
\begin{aligned}
& \pi_{2} \circ[\text { LHS of }(3.22)]_{U_{i}=1} \circ \iota_{1} \circ \pi_{1} \circ \eta_{\mathrm{op}}=\pi_{2} \circ[\text { RHS of }(3.22)]_{U_{i}=1} \circ \iota_{1} \circ \pi_{1} \circ \eta_{\mathrm{op}} \\
& =P_{A_{\mathrm{op}}^{(2)}}^{l} \circ \pi_{2} \circ \iota_{1} \circ \pi_{1} \circ \eta_{\mathrm{op}}=0 .
\end{aligned}
$$


On the other hand, using that $A_{\mathrm{cl}}$ is haploid, that $\tilde{\iota}_{\mathrm{cl}-\mathrm{op}}$ is an algebra map, and that $\tilde{\iota}_{\text {cl-op }}^{*}$ is a coalgebra map, one can check that the left-hand side of (3.28) is equal to $\lambda\left(\varepsilon_{\mathrm{op}} \circ \iota_{1} \circ \pi_{1} \circ \eta_{\mathrm{op}}\right) \pi_{2} \circ \eta_{\mathrm{op}}$ for some $\lambda \neq 0$. This implies that $\pi_{2} \circ \eta_{\mathrm{op}}=0$. Thus $\eta_{\mathrm{op}}=\sum_{i} \iota_{i} \circ \pi_{i} \circ \eta_{\mathrm{op}}=\iota_{1} \circ \pi_{1} \circ \eta_{\mathrm{op}}$. Hence, we have

$$
0 \neq \pi_{2} \circ \iota_{2}=\pi_{2} \circ m_{\mathrm{op}} \circ\left(\eta_{\mathrm{op}} \otimes \iota_{2}\right)=\pi_{2} \circ m_{\mathrm{op}} \circ\left(\left(\iota_{1} \circ \pi_{1} \circ \eta_{\mathrm{op}}\right) \otimes \iota_{2}\right) .
$$

However, the right-hand side is zero by (3.27). This is a contradiction and hence $A_{\text {op }}$ must be simple.

To formulate the next theorem we need the notion of the full centre of an algebra $[\mathrm{Fj}$, Def.4.9]. Recall that an algebra $A$ in a braided tensor category has a left centre and a right centre $[\mathrm{VZ}, \mathrm{O}]$, both of which are sub-algebras of $A$. Of these two, we will only need the left centre. The following definition is [Fr, Def. 2.31], which in our setting is equivalent to that of $[\mathrm{VZ}, \mathrm{O}]$.

Definition 3.17. Let $A$ be a symmetric special Frobenius algebra such that $m_{A} \circ \Delta_{A}=$ $\zeta_{A} \mathrm{id}_{A}$.

(i) The left centre $C_{l}(A)$ of $A$ is the image of the idempotent $\zeta_{A}^{-1} P_{A}^{l}$.

(ii) The full centre $Z(A)$ is $C_{l}(R(A))$.

That $\zeta_{A}^{-1} P_{A}^{l}$ is an idempotent follows from [FRS, Lem. 5.2] when keeping track of the factors $\zeta_{A}$ ([FRS] assumes normalised-special, i.e. $\zeta_{A}=1$ ). Note that $C_{l}(A)$ is again an object of $\mathcal{C}$, while $Z(A)$ is an object of $\mathcal{C}_{ \pm}^{2}$. Let $e_{A}^{l}: C_{l}(A) \rightarrow A$ be the embedding of $C_{l}(A)$ into $A$. The left centre is in fact the maximal subobject of $A$ such that

$$
m_{A} \circ c_{A, A} \circ\left(e_{A}^{l} \otimes \mathrm{id}_{A}\right)=m_{A},
$$

see [Fr, Lem. 2.32]. This observation explains the name left centre and also makes the connection to [O, Def. 15].

The full centre is by definition the image of the idempotent $\zeta_{A}^{-1} P_{R(A)}^{l}: R(A) \rightarrow$ $R(A)$. Since $\mathcal{C}_{ \pm}^{2}$ is abelian, the idempotent splits and we obtain the embedding and restriction morphisms

$$
e: Z(A) \hookrightarrow R(A) \text { and } r: R(A) \rightarrow Z(A)
$$

which obey $r \circ e=\operatorname{id}_{Z(A)}$ and $e \circ r=\zeta_{A}^{-1} P_{R(A)}^{l}$. It follows from Proposition 2.25 and [Fr, Prop. 2.37] that $Z(A)$ is a commutative symmetric Frobenius algebra in $\mathcal{C}_{ \pm}^{2}$ with structure morphisms ${ }^{5}$

$$
\begin{aligned}
& m_{Z(A)}=r \circ m_{R(A)} \circ(e \otimes e), \quad \eta_{Z(A)}=r \circ \eta_{R(A)}, \\
& \Delta_{Z(A)}=\zeta_{A} \cdot(r \otimes r) \circ \Delta_{R(A)} \circ e, \quad \varepsilon_{Z(A)}=\zeta_{A}^{-1} \cdot \varepsilon_{R(A)} \circ e .
\end{aligned}
$$

Moreover, if $A$ is simple then $Z(A)$ is simple, and if $A$ is simple and $\operatorname{dim} A \neq 0$, then $Z(A)$ is simple and special. The normalisation of the counit is such that

$$
\varepsilon_{Z(A)} \circ \eta_{Z(A)}=\zeta_{A}^{-2} \operatorname{dim} A \operatorname{DimC} .
$$

5 The normalisation of product and unit is the standard one. The factors in the coproduct and counit have to be included in order for $(A \mid Z(A), e)$ to be a Cardy algebra, see Theorem 3.18 below. The normalisation of the counit enters the Cardy condition (3.14) through the definition of $(\cdot)^{*}$. 
Theorem 3.18. Let $A$ be a special symmetric Frobenius $\mathcal{C}$-algebra. Then $(A \mid Z(A), e)$ is a $\operatorname{Cardy} \mathcal{C} \mid \mathcal{C}_{ \pm}^{2}$-algebra.

The proof of this theorem makes use the following two lemmas.

Lemma 3.19. $e: Z(A) \hookrightarrow R(A)$ is an algebra map, and $e^{*}=\zeta_{A} \cdot r$.

Proof. It follows from [Fr, Lem.2.29] (or by direct calculation, using in particular $\left.m_{R(A)} \circ \Delta_{R(A)}=\zeta_{A} \operatorname{id}_{R(A)}\right)$ that

$$
m_{R(A)} \circ(e \otimes e)=\zeta_{A}^{-1} \cdot P_{R(A)}^{l} \circ m_{R(A)} \circ(e \otimes e) .
$$

Substituting $e \circ r=\zeta_{A}^{-1} P_{R(A)}^{l}$ shows that $e$ is compatible with multiplication. For the unit one finds

$$
e \circ \eta_{Z(A)}=e \circ r \circ \eta_{R(A)}=\zeta_{A}^{-1} P_{R(A)}^{l} \circ \eta_{R(A)}=\eta_{R(A)} .
$$

Thus $e$ is an algebra map. For the second statement one computes

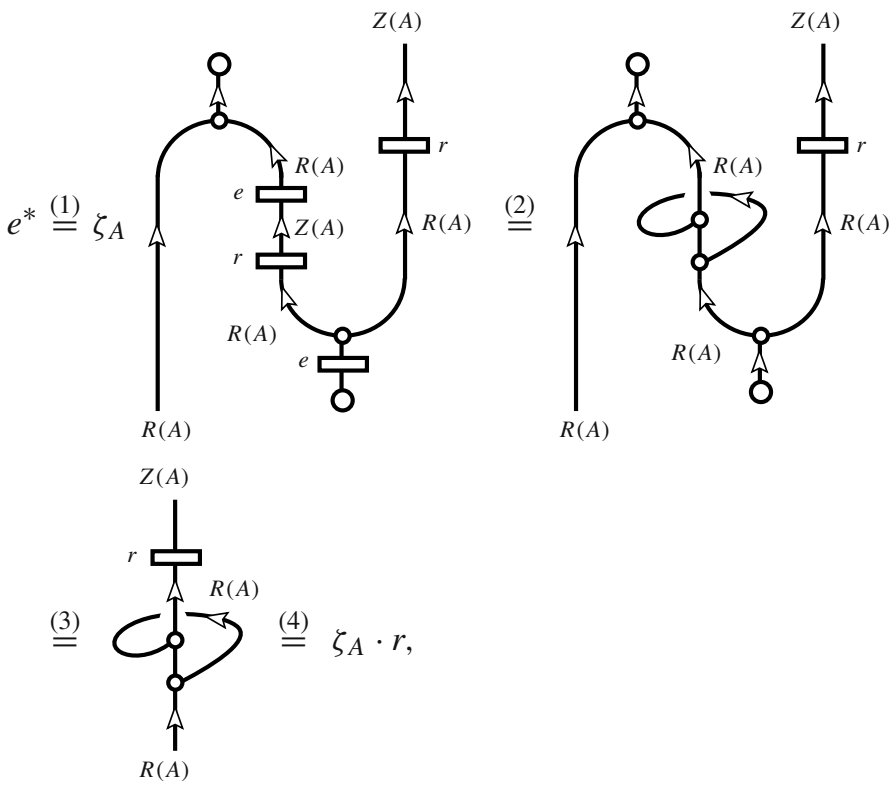

where in (1) the definitions (2.30) and (3.32) have been substituted, step (2) is $e$ o $r=\zeta_{A}^{-1} P_{R(A)}^{l}$, step (3) uses that $R(A)$ is symmetric Frobenius, and step (4) is again $e \circ r=\zeta_{A}^{-1} P_{R(A)}^{l}$.

Lemma 3.20. Let $A$ be a symmetric Frobenius algebra in $\mathcal{C}$. The morphism

$$
P_{R(A)}^{l} \circ m_{R(A)} \circ\left(P_{R(A)}^{l} \otimes P_{R(A)}^{l}\right): R(A) \otimes R(A) \longrightarrow R(A)
$$

is S-invariant.

The proof of this lemma is a slightly lengthy explicit calculation and has been deferred to Appendix A.2. 
Proof of Theorem 3.18. That $e$ is an algebra map was proved in Lemma 3.19. The centre condition (3.13) holds by property (3.30) of the left centre. The Cardy condition (3.14) also is an immediate consequence of Lemma 3.19,

$$
\iota_{\mathrm{cl}-\mathrm{op}} \circ \iota_{\mathrm{cl}-\mathrm{op}}^{*}=e \circ\left(\zeta_{A} r\right)=P_{R(A)}^{l} .
$$

The full centre $Z(A)$ is a commutative symmetric Frobenius algebra. It remains to prove modular invariance. That $\theta_{Z(A)}=\mathrm{id}_{Z(A)}$ is implied by commutativity and symmetry of $Z(A)$ [Fr, Prop. 2.25]. The S-invariance condition (3.3) follows from Lemma 3.20: In (3.37) substitute $P_{R(A)}^{l}=\zeta_{A} e \circ r$ and then put the resulting morphism into (3.3). Compose the resulting equation with $e \otimes \mathrm{id}_{W}$ from the right (i.e. from the bottom) and substitute the definition (3.32) of $m_{Z(A)}$. This results in the statement that $m_{Z(A)}$ is S-invariant.

The following theorem is analogous to [LR, Prop. 2.9] and [Fj, Thm.4.26], which, roughly speaking, answer the question under which circumstances the restriction of a two-dimensional conformal field theory to the boundary already determines the entire conformal field theory. The first work is set in Minkowski space and uses operator algebras and subfactors, while the second work is set in Euclidean space and uses modular tensor categories.

Theorem 3.21. Let $\left(A \mid A_{\mathrm{cl}}, \iota_{\mathrm{cl}-\mathrm{op}}\right)$ be a Cardy $\mathcal{C} \mid \mathcal{C}_{ \pm}^{2}$-algebra such that $\operatorname{dim} A \neq 0$ and $A_{\mathrm{cl}}$ is simple. Then $A$ is special and $\left(A \mid A_{\mathrm{cl}}, \iota_{\mathrm{cl}-\mathrm{op}}\right) \cong(A \mid Z(A), e)$ as Cardy algebras.

Proof. By Proposition 3.16, $A$ is simple and special. Since $A_{\mathrm{cl}}$ is simple, the algebra map $\iota_{\mathrm{cl}-\mathrm{op}}: A_{\mathrm{cl}} \rightarrow R(A)$ is either zero or a monomorphism. But $\iota_{\mathrm{cl}-\mathrm{op}} \circ \eta_{\mathrm{cl}}=\eta_{R(A)}$, and so $\iota_{\mathrm{cl}-\mathrm{op}} \neq 0$. Thus $\iota_{\mathrm{cl} \text {-op }}$ is monic. By Lemma 2.18(ii), $\zeta_{A}^{-1} \iota_{\mathrm{cl}-\mathrm{op}}^{*}$ is epi. The Cardy condition (3.14) implies

$$
\iota_{\mathrm{cl}-\mathrm{op}} \circ \zeta_{A}^{-1} \iota_{\mathrm{cl-op}}^{*}=\zeta_{A}^{-1} P_{R(A)}^{l}=e \circ r .
$$

Composing this with $e \circ r$ from the left yields $e \circ r \circ \iota_{\mathrm{cl}-\mathrm{op}} \circ \zeta_{A}^{-1} \iota_{\mathrm{cl}-\mathrm{op}}^{*}=e \circ r=$ $\iota_{\mathrm{cl}-\mathrm{op}} \circ \zeta_{A}^{-1} \iota_{\mathrm{cl}-\mathrm{op}}^{*}$. Since $\zeta_{A}^{-1} \iota_{\mathrm{cl}-\mathrm{op}}^{*}$ is epi, we have

$$
e \circ r \circ \iota_{\mathrm{cl}-\mathrm{op}}=\iota_{\mathrm{cl}-\mathrm{op}}
$$

Actually, (3.40) also follows from (3.13) and specialness of $R(A)$. We will prove that

$$
\left(f_{\mathrm{op}}, f_{\mathrm{cl}}\right):\left(A \mid A_{\mathrm{cl}}, \iota_{\mathrm{cl}-\mathrm{op}}\right) \longrightarrow(A \mid Z(A), e) \text { where } f_{\mathrm{op}}=\mathrm{id}_{A}, f_{\mathrm{cl}}=r \circ \iota_{\mathrm{cl}-\mathrm{op}},
$$

is an isomorphism of Cardy algebras.

$f_{\mathrm{cl}}$ is an algebra map: Compatibility with the units follows since $\iota_{\mathrm{cl}-\mathrm{op}}$ is an algebra map,

$$
f_{\mathrm{cl}} \circ \eta_{\mathrm{cl}}=r \circ \iota_{\mathrm{cl}-\mathrm{op}} \circ \eta_{\mathrm{cl}}=r \circ \eta_{R(A)}=\eta_{Z(A)} .
$$

Compatibility with the multiplication also follows since $\iota_{\mathrm{cl}-\mathrm{op}}$ is an algebra map,

$$
\begin{aligned}
& m_{Z(A)} \circ\left(f_{\mathrm{cl}} \otimes f_{\mathrm{cl}}\right)=r \circ m_{R(A)} \circ(e \otimes e) \circ(r \otimes r) \circ\left(\iota_{\mathrm{cl}-\mathrm{op}} \otimes \iota_{\mathrm{cl}-\mathrm{op}}\right) \\
& \quad=r \circ m_{R(A)} \circ\left(\iota_{\mathrm{cl}-\mathrm{op}} \otimes \iota_{\mathrm{cl}-\mathrm{op}}\right)=r \circ \iota_{\mathrm{cl}-\mathrm{op}} \circ m_{\mathrm{cl}}=f_{\mathrm{cl}} \circ m_{\mathrm{cl}},
\end{aligned}
$$

where in the second step we used (3.40). 
$f_{\mathrm{cl}}$ is an isomorphism: As above, since $f_{\mathrm{cl}}$ is an algebra map and since $A_{\mathrm{cl}}$ is simple, $f_{\mathrm{cl}}$ has to be monic. By Lemma 3.19, $r^{*}=\zeta_{A}^{-1} e$. Thus $f_{\mathrm{cl}}^{*}=\iota_{\mathrm{cl}-\mathrm{op}}^{*} \circ r^{*}=\zeta_{A}^{-1} \iota_{\mathrm{cl}-\mathrm{op}}^{*} \circ e$ and

$$
f_{\mathrm{cl}} \circ f_{\mathrm{cl}}^{*}=r \circ \iota_{\mathrm{cl}-\mathrm{op}} \circ \zeta_{A}^{-1} \iota_{\mathrm{cl}-\mathrm{op}}^{*} \circ e=r \circ e \circ r \circ e=\mathrm{id}_{Z(A)},
$$

and so $f_{\mathrm{cl}}$ is also epi, and hence iso.

$f_{\mathrm{cl}}$ is a coalgebra map: Since $f_{\mathrm{cl}}$ is an algebra map, so is $f_{\mathrm{cl}}^{-1}$. By (3.44), $f_{\mathrm{cl}}^{-1}=f_{\mathrm{cl}}^{*}$ and by Lemma 2.18 (iii) this implies that $f_{\mathrm{cl}}$ is a also coalgebra map.

The diagram (3.15) commutes: Commutativity of (3.15) is equivalent to $e \circ f_{\mathrm{cl}}=\iota_{\mathrm{cl}-\mathrm{op}}$, which holds by (3.40).

Let $A$ be a special symmetric Frobenius algebra. So far we have seen that $(A \mid Z(A), e)$ is a Cardy algebra, and that all Cardy algebras with $A_{\mathrm{op}}=A$ and simple $A_{\mathrm{cl}}$ are of this form. It is now natural to ask if every simple $A_{\mathrm{cl}}$ does occur as part of a Cardy algebra. The following theorem provides an affirmative answer. Recall that for an $A$-left module $M$, the object $M^{\vee} \otimes_{A} M$ is an algebra (see e.g. [KR, Lem. 4.2]).

Theorem 3.22. If $A_{\mathrm{cl}}$ is a simple modular invariant commutative symmetric Frobenius $\mathcal{C}_{ \pm}^{2}$-algebra, then there exist a simple special symmetric Frobenius $\mathcal{C}$-algebra $A$ and $a$ morphism $\iota_{\mathrm{cl}-\mathrm{op}}: A_{\mathrm{cl}} \rightarrow R(A)$ such that

(i) $A_{\mathrm{cl}} \cong Z(A)$ as Frobenius algebras;

(ii) $\left(A \mid A_{\mathrm{cl}}, \iota_{\mathrm{cl}-\mathrm{op}}\right)$ is a Cardy $\mathcal{C} \mid \mathcal{C}_{ \pm}^{2}$-algebra;

(iii) $T\left(A_{\mathrm{cl}}\right) \cong \bigoplus_{\kappa \in \mathcal{J}} M_{\kappa}^{\vee} \otimes_{A} M_{\kappa}$ as algebras, where $\left\{M_{\kappa}\right\}_{\kappa \in \mathcal{J}}$ is a set of representatives of the isomorphism classes of simple A-left modules.

Proof. By Remark 3.6, we have $\operatorname{dim} A_{\mathrm{cl}}=Z_{00} \operatorname{Dim} \mathcal{C} \neq 0$, and by Lemma 2.11, $A_{\mathrm{cl}}$ is haploid. It then follows from Theorem 3.4 that $A_{\mathrm{cl}}$ is special. By Proposition 2.24, $T\left(A_{\mathrm{cl}}\right)$ is a special symmetric Frobenius algebra in $\mathcal{C}$. Thus $T\left(A_{\mathrm{cl}}\right)=\bigoplus_{i} A_{i}$, where the $A_{i}$ are simple symmetric Frobenius algebras. We will show that at least one of the $A_{i}$ is special. Since $T\left(A_{\mathrm{cl}}\right)$ is special, we have $m_{T\left(A_{\mathrm{cl}}\right)} \circ \Delta_{T\left(A_{\mathrm{cl}}\right)}=\zeta \mathrm{id}_{T\left(A_{\mathrm{cl}}\right)}$ for some $\zeta \in \mathbb{C}^{\times}$. Restricting this to the summand $A_{i}$ shows $m_{i} \circ \Delta_{i}=\zeta \operatorname{id}_{A_{i}}$. Furthermore, $\varepsilon_{T\left(A_{\mathrm{cl}}\right)} \circ \eta_{T\left(A_{\mathrm{cl}}\right)}=\xi \mathrm{id}_{\mathbf{1}}$ for some $\xi \in \mathbb{C}^{\times}$. But $\varepsilon_{T\left(A_{\mathrm{cl}}\right)} \circ \eta_{T\left(A_{\mathrm{cl}}\right)}=\sum_{i} \varepsilon_{i} \circ \eta_{i}$, and so at least one of the $\varepsilon_{i} \circ \eta_{i}$ has to be nonzero. Therefore, at least one of the $A_{i}$ is special; let $A \equiv A_{i}$ be this summand. We denote the embedding $A \hookrightarrow T\left(A_{\mathrm{cl}}\right)$ by $e_{0}$ and the restriction $T\left(A_{\mathrm{cl}}\right) \rightarrow A$ by $r_{0}$. Notice that $r_{0}$ is an algebra homomorphism. Define

$$
\iota_{\mathrm{cl}-\mathrm{op}}=\hat{\chi}\left(r_{0}\right): A_{\mathrm{cl}} \longrightarrow R(A) .
$$

By Proposition 2.16, $\iota_{\mathrm{cl}-\mathrm{op}}$ is an algebra homomorphism. Next we verify the centre condition (3.13), or rather its equivalent form (3.20). By substituting the definitions, one can convince oneself that the commutativity $m_{\mathrm{cl}} \circ c_{A_{\mathrm{cl}}, A_{\mathrm{cl}}}=m_{\mathrm{cl}}$ of $A_{\mathrm{cl}}$ in $\mathcal{C}_{ \pm}^{2}$ implies the condition $m_{T\left(A_{\mathrm{cl}}\right)} \circ \Gamma=m_{T\left(A_{\mathrm{cl}}\right)}$ in $\mathcal{C}$, see [K2, Prop. 3.6]. Here, $\Gamma: T\left(A_{\mathrm{cl}}\right) \otimes T\left(A_{\mathrm{cl}}\right) \rightarrow$ $T\left(A_{\mathrm{cl}}\right) \otimes T\left(A_{\mathrm{cl}}\right)$ is given by

$$
\Gamma=\bigoplus_{m, n}\left(\operatorname{id}_{C_{n}^{l}} \otimes c_{C_{m}^{l}, C_{n}^{r}} \otimes \operatorname{id}_{C_{m}^{r}}\right) \circ\left(c_{C_{m}^{l}, C_{n}^{l}} \otimes c_{C_{n}^{r}, C_{m}^{r}}^{-1}\right) \circ\left(\operatorname{id}_{C_{m}^{l}} \otimes c_{C_{n}^{l}, C_{m}^{r}}^{-1} \otimes \operatorname{id}_{C_{n}^{r}}\right),
$$

and we decomposed $A_{\mathrm{cl}}$ as $A_{\mathrm{cl}}=\oplus_{n} C_{n}^{l} \times C_{n}^{r}$. As a consequence we obtain the identity

$$
r_{0} \circ m_{T\left(A_{\mathrm{cl}}\right)} \circ \Gamma \circ\left(\mathrm{id}_{A_{\mathrm{cl}}} \otimes e_{0}\right)=r_{0} \circ m_{T\left(A_{\mathrm{cl}}\right)} \circ\left(\mathrm{id}_{A_{\mathrm{cl}}} \otimes e_{0}\right) \text {. }
$$


Using that $r_{0}$ is an algebra map, and that by definition $\tilde{\iota}_{\mathrm{cl}-\mathrm{op}}=r_{0}$, we obtain (3.20).

In order to show that $\left(A \mid A_{\mathrm{cl}}, \iota_{\mathrm{cl}-\mathrm{op}}\right)$ is a Cardy algebra, it remains to show that the Cardy condition (3.14) is satisfied. We will demonstrate this via a detour by first proving that $A_{\mathrm{cl}} \cong Z(A)$ as Frobenius algebras.

Recall the notations $e$ and $r$ given in (3.31). Using the centre condition (3.20) one can check that $P_{R(A)}^{l} \circ \iota_{\mathrm{cl}-\mathrm{op}}=m_{R(A)} \circ \Delta_{R(A)} \circ \iota_{\mathrm{cl}-\mathrm{op}}$. By specialness of $A$ we have $m_{A} \circ \Delta_{A}=\zeta_{A} \mathrm{id}_{A}$ and so together with $e \circ r=\zeta_{A}^{-1} P_{R(A)}^{l}$ we get,

$$
e \circ r \circ \iota_{\mathrm{cl}-\mathrm{op}}=\iota_{\mathrm{cl}-\mathrm{op}}
$$

Next, consider the morphism

$$
f_{\mathrm{cl}}=r \circ \iota_{\mathrm{cl}-\mathrm{op}}: A_{\mathrm{cl}} \longrightarrow Z(A) .
$$

By the same derivation as in (3.42) and (3.43) one sees that $f_{\mathrm{cl}}$ is an algebra map. In particular, $f_{\mathrm{cl}} \circ \eta_{\mathrm{cl}}=\eta_{Z(A)} \neq 0$ and so $f_{\mathrm{cl}} \neq 0$. Since $A_{\mathrm{cl}}$ is simple, $f_{\mathrm{cl}}$ has to be a monomorphism.

By the same argument as used in the proof of Theorem 3.4(ii), up to isomorphism $A_{\mathrm{cl}}$ is the unique simple local $A_{\mathrm{cl}}$ (left-)module. The algebra monomorphism $f_{\mathrm{cl}}$ turns $Z(A)$ into an $A_{\mathrm{cl}}$-module. Since $Z(A)$ is commutative, it is local as an $A_{\mathrm{cl}}$-module, and so $Z(A) \cong A_{\mathrm{cl}}^{\oplus N}$ for some $N \geq 1$. By construction, $A$ is a simple special symmetric Frobenius algebra. Proposition 2.25 and Corollary 2.27 show that $R(A)$ inherits all these properties, and thus $Z(A)$ is simple (see the comment below Eq. (3.32)). By Theorem 3.18, $Z(A)$ is modular invariant, and then by Theorem 3.4(i), $\operatorname{dim} Z(A)=\operatorname{Dim} \mathcal{C}$. This implies that $N=1$ in $Z(A) \cong A_{\mathrm{cl}}^{\oplus N}$, and so $f_{\mathrm{cl}}$ is in fact an isomorphism.

Since $A_{\mathrm{cl}}$ and $Z(A)$ are both haploid, we have $\varepsilon_{Z(A)} \circ f_{\mathrm{cl}}=\xi \varepsilon_{\mathrm{cl}}$ for some $\xi \in \mathbb{C}^{\times}$. The counit uniquely determines the Frobenius structure on $A_{\mathrm{cl}}$ and $Z(A)$ (see e.g. [FRS, Lemma 3.7]), so that $f_{\mathrm{cl}}$ is a coalgebra isomorphism iff $\xi=1$. To compute $\xi$ we compose the above identity with $\eta_{\mathrm{cl}}$ from the right. Defining $\zeta_{\mathrm{cl}}$ via $\varepsilon_{\mathrm{cl}} \circ \eta_{\mathrm{cl}}=\zeta_{\mathrm{cl}}^{-1} \operatorname{Dim} \mathcal{C} \cdot \mathrm{id}_{1}$ and using (3.33) gives $\xi=\operatorname{dim} A \zeta_{\mathrm{cl}} / \zeta_{A}^{2}$. By rescaling the comultiplication and the counit of $A$, and consequently changing $\zeta_{A}$, we can always achieve $\xi=1$. This proves part (i) of the theorem.

Equation (3.48) implies that $\iota_{\mathrm{cl}-\mathrm{op}}=e \circ f_{\mathrm{cl}}$. Since $f_{\mathrm{cl}}$ is an isomorphism of Frobenius algebras, by Lemmas 2.18 and 3.19 we have

$$
\iota_{\mathrm{cl}-\mathrm{op}} \circ \iota_{\mathrm{cl}-\mathrm{op}}^{*}=e \circ f_{\mathrm{cl}} \circ f_{\mathrm{cl}}^{*} \circ e^{*}=\zeta_{A} e \circ r=P_{R(A)}^{l} .
$$

Thus $\left(A \mid A_{\mathrm{cl}}, \iota_{\mathrm{cl}-\mathrm{op}}\right)$ is a Cardy algebra. This proves part (ii) of the theorem.

Part (iii) can be seen as follows. By [KR, Prop. 4.3], $T Z(A) \cong \oplus_{\kappa \in \mathcal{J}} M_{\kappa}^{\vee} \otimes_{A} M_{\kappa}$ as algebras. Together with the observation that $T\left(f_{\mathrm{cl}}\right): T\left(A_{\mathrm{cl}}\right) \rightarrow T Z(A)$ is an isomorphism of algebras, this proves part (iii).

Remark 3.23. Part (i) of Theorem 3.22 was announced by Müger [Mü2]. We provide an independent proof in the setting of Cardy algebras

The above theorem, together with Lemma 2.11 and Theorem 3.4, shows that a simple commutative symmetric Frobenius $\mathcal{C}_{ \pm}^{2}$-algebra $A_{\mathrm{cl}}$ with $\operatorname{dim} A_{\mathrm{cl}}=\operatorname{Dim} \mathcal{C}$ is always part of a Cardy algebra $\left(A_{\mathrm{op}} \mid A_{\mathrm{cl}}, \iota_{\mathrm{cl}-\mathrm{op}}\right)$ for some simple special symmetric Frobenius algebra $A_{\text {op }}$ in $\mathcal{C}$. However, the above proof also illustrates that $A_{\text {op }}$ is not unique. This raises the question how two Cardy algebras with a given $A_{\mathrm{cl}}$ can differ. This question is answered by [KR, Thm. 1.1], which in the present framework can be restated as follows. 
Theorem 3.24. If $\left(A_{\mathrm{op}}^{(i)} \mid A_{\mathrm{cl}}^{(i)}, \iota_{\mathrm{cl}-\mathrm{op}}^{(i)}\right), i=1,2$ are two Cardy $\mathcal{C} \mid \mathcal{C}_{ \pm}^{2}$-algebras such that $A_{\mathrm{cl}}^{(i)}$ is simple and $\operatorname{dim} A_{\mathrm{op}}^{(i)} \neq 0$ for $i=1,2$, then $A_{\mathrm{cl}}^{(1)} \cong A_{\mathrm{cl}}^{(2)}$ as algebras if and only if $A_{\mathrm{op}}^{(1)}$ and $A_{\mathrm{op}}^{(2)}$ are Morita equivalent.

Proof. Theorem $1.1 \mathrm{in}[\mathrm{KR}]$ is stated for $A_{\mathrm{op}}^{(i)}$ being non-degenerate algebras and $A_{\mathrm{cl}}^{(i)}=$ $Z\left(A_{\mathrm{op}}^{(i)}\right)$ for $i=1,2$. By Proposition 3.16, $A_{\mathrm{op}}^{(i)}$ are simple and special for $i=1,2$. Then by [KR, Lem.2.1], $A_{\mathrm{op}}^{(i)}$ are non-degenerate algebras. By Theorem 3.21, we have $A_{\mathrm{cl}}^{(i)} \cong Z\left(A_{\mathrm{op}}^{(i)}\right)$ as Frobenius algebras. Finally, by [KR, Thm. 1.1], $Z\left(A_{\mathrm{op}}^{(1)}\right) \cong Z\left(A_{\mathrm{op}}^{(2)}\right)$ as algebras iff $A_{\mathrm{op}}^{(1)}$ and $A_{\mathrm{op}}^{(2)}$ are Morita equivalent.

Let $C_{\max }\left(\mathcal{C}_{ \pm}^{2}\right)$ be the set of equivalence classes $[B]$ of simple modular invariant commutative symmetric Frobenius algebras $B$ in $\mathcal{C}_{ \pm}^{2}$. Two such algebras $B$ and $B^{\prime}$ are equivalent if $B$ and $B^{\prime}$ are isomorphic as algebras (but not necessarily as Frobenius algebras). Let $M_{\text {simp }}(\mathcal{C})$ be the set of Morita classes of simple special symmetric Frobenius algebras in $\mathcal{C}$. Define the map $z: M_{\text {simp }}(\mathcal{C}) \rightarrow C_{\max }\left(\mathcal{C}_{ \pm}^{2}\right)$ by $z:\{A\} \rightarrow[Z(A)]$, where $\{A\}$ denotes the Morita class of $A$. From Theorem 3.22(i) and [KR, Thm. 1.1] we learn:

Corollary 3.25. The map $z: M_{\text {simp }}(\mathcal{C}) \rightarrow C_{\max }\left(\mathcal{C}_{ \pm}^{2}\right)$ is a bijection.

\section{A. Appendix}

A.1. Proof of Lemma 2.7. We will show that if $\left(F, \psi_{2}^{F}, \psi_{0}^{F}\right)$ is a colax tensor functor from $\mathcal{C}_{1}$ to $\mathcal{C}_{2}$, then $\left(G, \phi_{2}^{G}, \phi_{0}^{G}\right)$ is a lax tensor functor from $\mathcal{C}_{2}$ to $\mathcal{C}_{1}$. Applying this result to the opposed categories then gives the converse statement.

We need to show that $\phi_{0}^{G}$ and $\phi_{2}^{G}$ make the diagrams (2.1) and (2.2) commute. We first prove the commutativity of (2.1). Consider the following diagram:

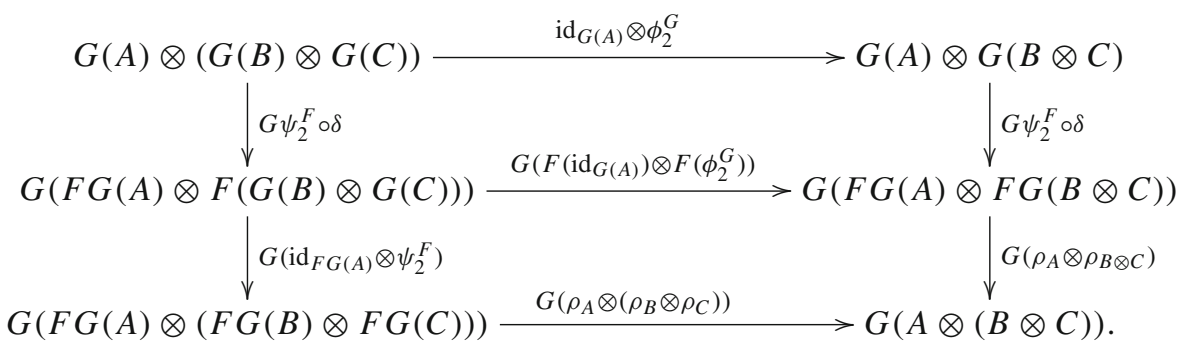

The top subdiagram is commutative because of the naturality of $G \psi_{2}^{F} \circ \delta$. The commutativity of the bottom subdiagram follows from the following identities:

$$
\begin{aligned}
\left(\rho_{B} \otimes \rho_{C}\right) \circ \psi_{2}^{F} & =\left(\rho_{B} \otimes \rho_{C}\right) \circ \psi_{2}^{F} \circ \rho F \circ F \delta \\
& =\rho_{B \otimes C} \circ F G\left(\rho_{B} \otimes \rho_{C}\right) \circ F G\left(\psi_{2}^{F}\right) \circ F \delta \\
& =\rho_{B \otimes C} \circ F\left(\phi_{2}^{G}\right)
\end{aligned}
$$

as a map $F(G(B) \otimes G(C)) \rightarrow B \otimes C$. The commutativity of (A.1) implies that the composition of maps in the left column in (2.1) can be replaced by

$$
G\left(\rho_{A} \otimes\left(\rho_{B} \otimes \rho_{C}\right)\right) \circ G\left(\left(\operatorname{id}_{F G(A)} \otimes \psi_{2}^{F}\right) \circ \psi_{2}^{F}\right) \circ \delta .
$$


Similarly, we can show that the composition of maps in the right column in (2.1) can be replaced by

$$
G\left(\left(\rho_{A} \otimes \rho_{B}\right) \otimes \rho_{C}\right) \circ G\left(\left(\psi_{2}^{F} \otimes \operatorname{id}_{F G(C)}\right) \circ \psi_{2}^{F}\right) \circ \delta .
$$

Using the commutativity of (2.3), it is easy to see that (2.1) with the left and right columns of (2.1) replaced by (A.3) and (A.4) respectively is commutative. Hence (2.1) is commutative.

Now we prove the commutativity of the first diagram in (2.2).

$$
\begin{aligned}
\phi_{2}^{G} \circ\left(\phi_{0}^{G} \otimes \mathrm{id}_{G(A)}\right) \\
\quad \stackrel{(1)}{=} G\left(\rho_{\mathbf{1}_{2}} \otimes \rho_{A}\right) \circ G \psi_{2}^{F} \circ \delta \circ\left[\left(G \psi_{0}^{F} \circ \delta_{\mathbf{1}_{1}}\right) \otimes \mathrm{id}_{G(A)}\right] \\
\quad \stackrel{(2)}{=} G\left(\rho_{\mathbf{1}_{2}} \otimes \rho_{A}\right) \circ G \psi_{2}^{F} \circ G F\left(G \psi_{0} \otimes \mathrm{id}_{G(A)}\right) \circ G F\left(\delta_{\mathbf{1}_{1}} \otimes \mathrm{id}_{G(A)}\right) \circ \delta \\
\quad \stackrel{(3)}{=} G\left(\rho_{\mathbf{1}_{2}} \otimes \rho_{A}\right) \circ G\left(F G\left(\psi_{0}^{F}\right) \otimes \mathrm{id}_{F G(A)}\right) \circ G \psi_{2}^{F} \circ G F\left(\delta_{\mathbf{1}_{1}} \otimes \mathrm{id}_{G(A)}\right) \circ \delta \\
\quad \stackrel{(4)}{=} G\left(\mathrm{id}_{\mathbf{1}_{2}} \otimes \rho_{A}\right) \circ G\left(\left[\psi_{0}^{F} \circ \rho_{F\left(\mathbf{1}_{1}\right)} \circ(F \delta)_{\mathbf{1}_{1}}\right] \otimes \mathrm{id}_{F G(A)}\right) \circ G \psi_{2}^{F} \circ \delta \\
\quad \stackrel{(5)}{=} G\left(\mathrm{id}_{\mathbf{1}_{2}} \otimes \rho_{A}\right) \circ G\left(\psi_{0}^{F} \otimes \mathrm{id}_{F G(A)}\right) \circ G \psi_{2}^{F} \circ \delta \\
\quad \stackrel{(6)}{=} G\left(\mathrm{id}_{\mathbf{1}_{2}} \otimes \rho_{A}\right) \circ G\left(l_{F G(A)}^{-1}\right) \circ G F\left(l_{G(A)}\right) \circ \delta \\
\quad \stackrel{(7)}{=} G\left(l_{A}^{-1}\right) \circ G \rho_{A} \circ \delta G \circ l_{G(A)} \\
\quad \stackrel{(8)}{=} G\left(l_{A}^{-1}\right) \circ l_{G(A)},
\end{aligned}
$$

where in step (1) we substituted the definition of $\phi_{0}^{G}, \phi_{2}^{G}$ given in (2.10); in step (2) we used the naturality of $\delta$; in step (3) we used the naturality of $G \psi_{2}^{F}$; in step (4) we switched the position between $G \rho_{\mathbf{1}_{2}}$ and $G F G\left(\psi_{0}^{F}\right)$ and the position between $G \psi_{2}$ and $G F\left(\delta_{\mathbf{1}_{2}} \otimes \mathrm{id}_{G(A)}\right)$ using the naturality of $\rho$ and $F \psi_{2}^{G}$ respectively; in step (5) we applied the second identity in (2.8); in step (6) we used (2.4); in step (7) we used the naturality of $l^{-1}$ and $\delta$; in step (8) we used the first identity in (2.8).

The proof of the commutativity of the second diagram in (2.2) is similar. Thus we have shown that $G$ is a lax tensor functor.

A.2. Proof of Lemma 3.20. To prepare the proof, recall that for a given object $B \in \mathcal{C}$, the modular group $P S L(2, \mathbb{Z})$ acts on the space $\oplus_{i} \operatorname{Hom}_{\mathcal{C}}\left(B \otimes U_{i}, U_{i}\right)$, see e.g. [BK, Sect 3.1] and [K3, Eq. (4.55)]. We will only need the action of $S$ and $S^{-1}$. Let $f \in$ $\oplus_{i} \operatorname{Hom}_{\mathcal{C}}\left(B \otimes U_{i}, U_{i}\right)$. Then
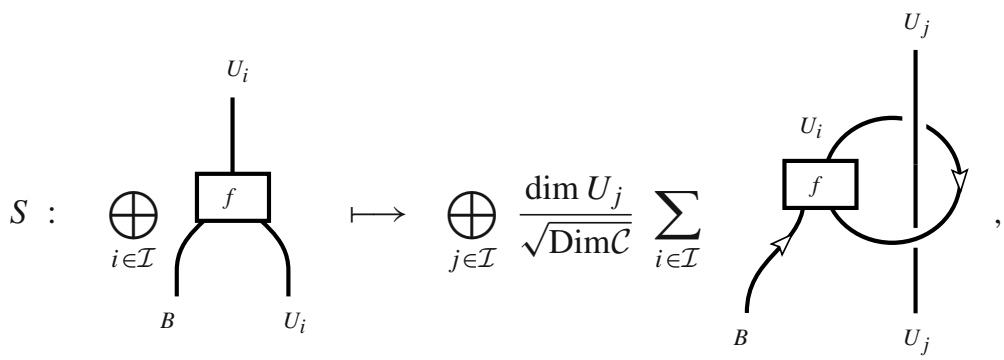

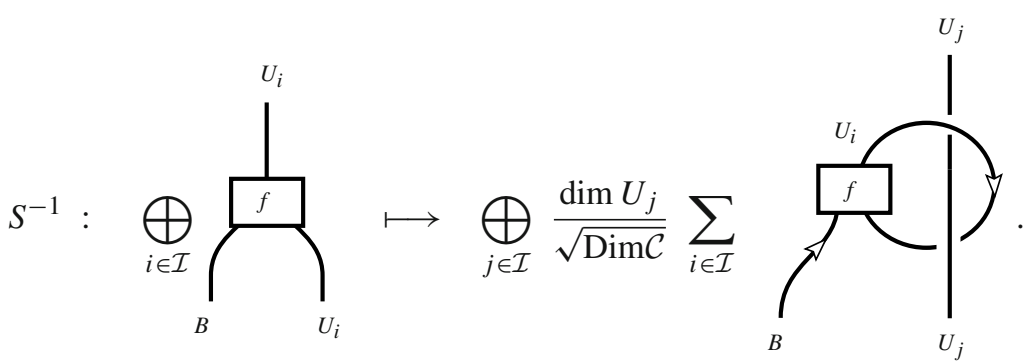

By Lemma 3.2, to establish that (3.37) is S-invariant, it is enough to prove the identity (3.4) when $f$ is given by (3.37). Using (A.6) and (A.7), we can see that Eq. (3.4) simply says that $\oplus_{i, j}$ [RHS of (3.4)] is invariant under the action of $S \times S$. Consider the element $g$ of $\oplus_{j, k \in \mathcal{I}} \operatorname{Hom}_{\mathcal{C}_{ \pm}^{2}}\left(R(A) \otimes\left(U_{j}^{\vee} \times U_{k}\right), U_{j}^{\vee} \times U_{k}\right)$ given by

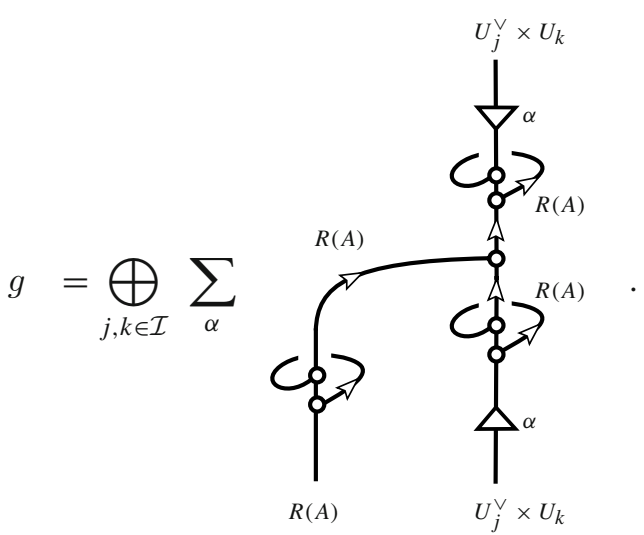

By the above arguments, proving $S$-invariance of (3.37) is equivalent to proving invariance of $g$ under the action of $S \times S$.

For $i \in \mathcal{I}$, we denote by $g_{i}$ the component of $g$ in

$$
\left(\oplus_{j \in \mathcal{I}} \operatorname{Hom}_{\mathcal{C}}\left(A \otimes U_{i}^{\vee} \otimes U_{j}^{\vee}, U_{j}^{\vee}\right)\right) \otimes\left(\oplus_{k \in \mathcal{I}} \operatorname{Hom}_{\mathcal{C}}\left(U_{i} \otimes U_{k}, U_{k}\right)\right) .
$$

We view the second Hom-space in the above tensor product as a Hom-space in $\mathcal{C}_{+}$instead of $\mathcal{C}_{-}$. It is enough to show that $g_{i}$ is invariant under the action of $S \times S^{-1}$. Note that the action of $S^{-1}$ in $\mathcal{C}_{+}$is equivalent to that of $S$ in $\mathcal{C}_{-}$.

The morphism $g_{i}$ can be canonically identified with a bilinear pairing

$$
\begin{aligned}
& (\cdot, \cdot)_{i}:\left(\bigoplus_{j \in \mathcal{I}} \operatorname{Hom}_{\mathcal{C}}\left(U_{j}^{\vee}, A \otimes U_{i}^{\vee} \otimes U_{j}^{\vee}\right)\right) \\
& \quad \times\left(\bigoplus_{k \in \mathcal{I}} \operatorname{Hom}_{\mathcal{C}}\left(U_{k}, U_{i} \otimes U_{k}\right)\right) \longrightarrow \mathbb{C}
\end{aligned}
$$

as follows. For $h_{1} \in \operatorname{Hom}_{\mathcal{C}}\left(U_{j}^{\vee}, A \otimes U_{i}^{\vee} \otimes U_{j}^{\vee}\right)$ and $h_{2} \in \operatorname{Hom}_{\mathcal{C}}\left(U_{k}, U_{i} \otimes U_{k}\right)$ we set

$$
\left(h_{1}, h_{2}\right)_{i}=\left(\operatorname{dim} U_{j} \operatorname{dim} U_{k}\right)^{-1} \operatorname{tr}_{U_{j}^{\vee} \times U_{k}}\left[g_{i} \circ\left(h_{1} \times h_{2}\right)\right] .
$$


When substituting the explicit form of the product $m_{R(A)}$ of $R(A)=(A \times \mathbf{1}) \otimes R(\mathbf{1})$, after a short calculation one finds

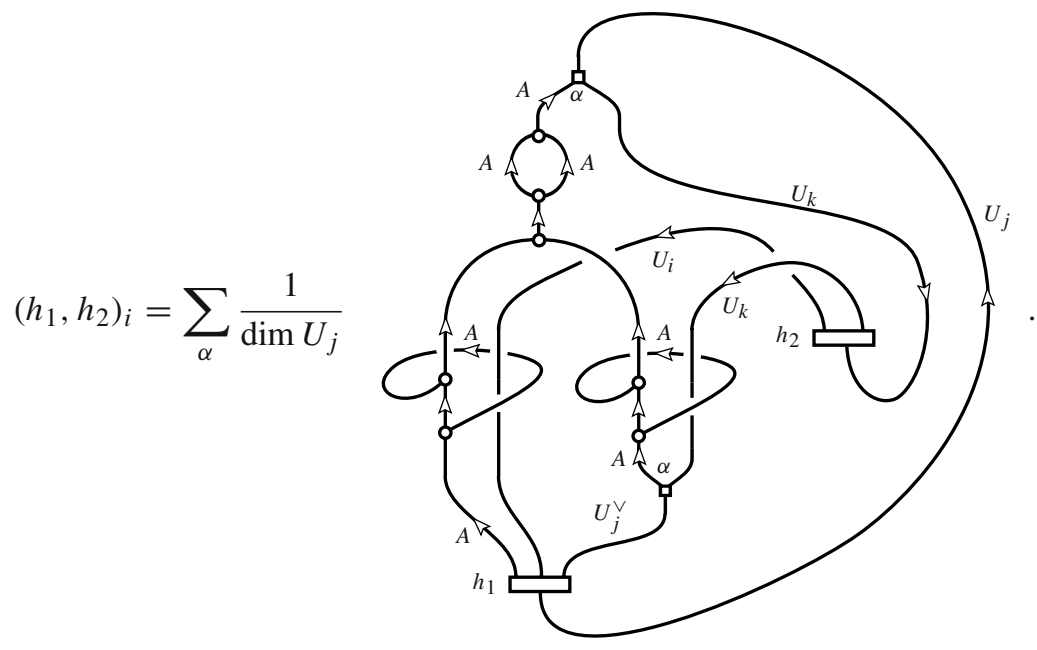

Here the top morphism $P_{R(A)}^{l}$ has been simplified with the help of the identity

$$
\begin{aligned}
& P_{R(A)} \circ m_{R(A)} \circ\left(P_{R(A)} \otimes P_{R(A)}\right) \\
& \quad=\left(\bigoplus_{i \in \mathcal{I}}\left(\left(m_{A} \circ \Delta_{A}\right) \otimes \mathrm{id}_{U_{i}^{\vee}}\right) \times \mathrm{id}_{U_{i}}\right) \circ m_{R(A)} \circ\left(P_{R(A)} \otimes P_{R(A)}\right),
\end{aligned}
$$

which can be checked by direct calculation along the same lines as in the proof of [Fr, Lem 3.10].

The action of the modular transformation $S$ on $\oplus_{i \in \mathcal{I}}\left(B \otimes U_{i}, U_{i}\right)$ for $B \in \mathcal{C}$ naturally induces an action on $\bigoplus_{i \in \mathcal{I}}\left(U_{i}, B \otimes U_{i}\right)$ [K3, Prop. 5.14], which we denote by $S^{*}$. In the present case we get an action of $S^{*}$ on $\oplus_{j \in \mathcal{I}} \operatorname{Hom}_{\mathcal{C}}\left(U_{j}^{\vee}, A \otimes U_{i}^{\vee} \otimes U_{j}^{\vee}\right)$ and $\oplus_{k \in \mathcal{I}} \operatorname{Hom}_{\mathcal{C}}\left(U_{k}, U_{i} \otimes U_{k}\right)$. Then to show $g_{i}$ is invariant under the action of $S \times S^{-1}$ amounts to showing that

$$
\left(h_{1}, h_{2}\right)_{i}=\left(\left(S^{-1}\right)^{*} h_{1}, S^{*} h_{2}\right)_{i},
$$


for all $h_{1} \in \operatorname{Hom}_{\mathcal{C}}\left(U_{j}^{\vee}, A \otimes U_{i}^{\vee} \otimes U_{j}^{\vee}\right)$ and $h_{2} \in \operatorname{Hom}_{\mathcal{C}}\left(U_{k}, U_{i} \otimes U_{k}\right)$. We have

$\left(\left(S^{-1}\right)^{*} h_{1}, S^{*} h_{2}\right)_{i}=\sum_{m, n, \alpha} \frac{\operatorname{dim} U_{n}}{\operatorname{Dim} \mathcal{C}}$

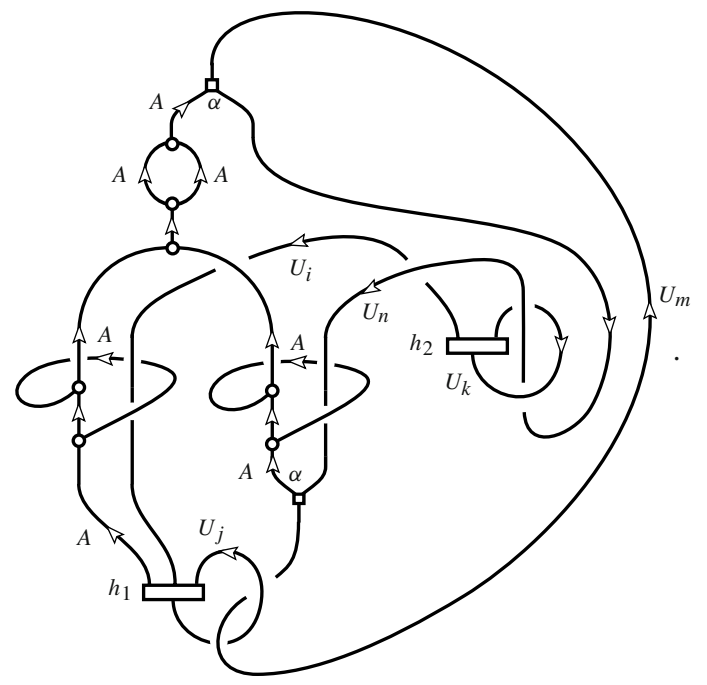

(A.14)

Now drag the upper vertex indexed by $\alpha$ in the above graph along its $U_{m}^{\vee}$-leg until it meets the lower vertex also indexed by $\alpha$, then sum over $\alpha$ and $m$. This gives

$$
\left(\left(S^{-1}\right)^{*} h_{1}, S^{*} h_{2}\right)_{i}=\sum_{n} \frac{\operatorname{dim} U_{n}}{\operatorname{Dim} \mathcal{C}}
$$

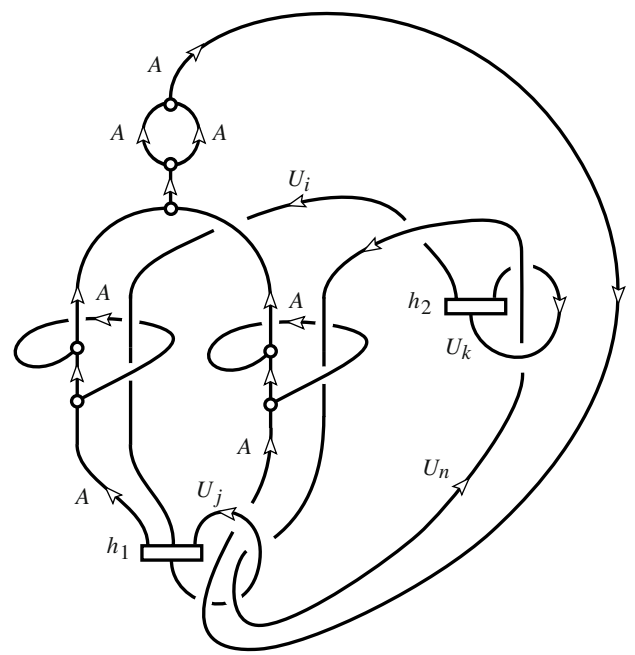


If we just look at the neighbourhood of the $U_{n}$-loop in the above graph, we see the following subgraphs:
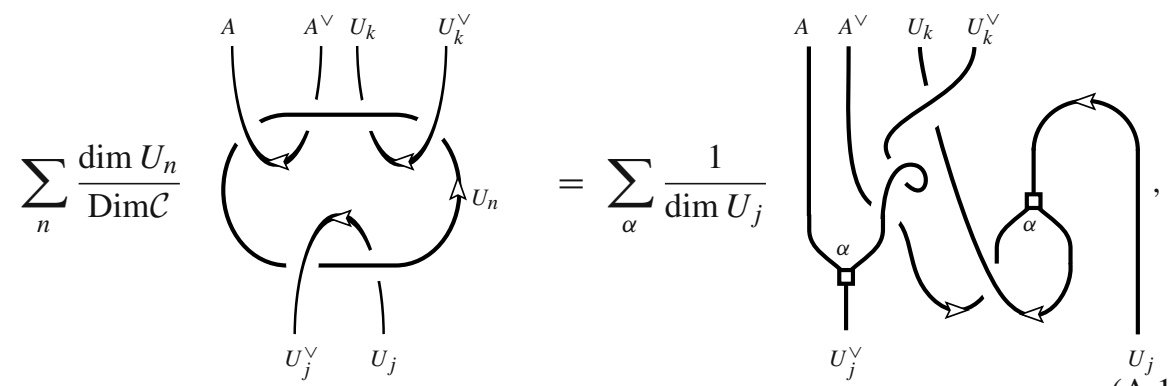

(A.16)

where we have applied [BK, Cor. 3.1.11]. Substituting this subgraph back to the original graph in (A.15), we obtain

$\left(\left(S^{-1}\right)^{*} h_{1}, S^{*} h_{2}\right)_{i}=\sum_{\alpha} \frac{1}{\operatorname{dim} U_{j}}$

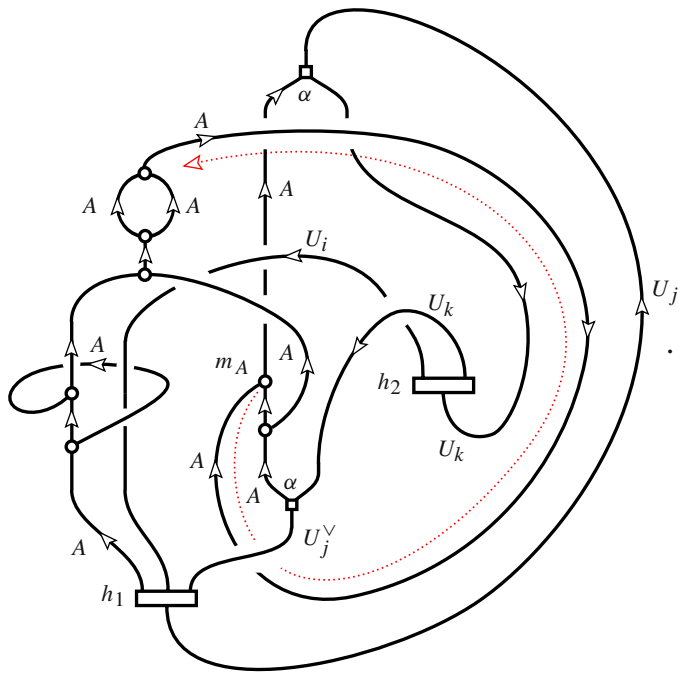

(A.17)

The graph in (A.17) is equal to that in (A.11). In order to see this, we first drag the "bubble" $\left(m_{A} \circ \Delta_{A}\right)$ along $A$ lines and through the $m_{A}$ vertex (because $m_{A} \circ \Delta_{A}$ is a bimodule map) until it reaches the lower-left leg of the upper vertex indexed by $\alpha$. Then drag the $m_{A}$ vertex along the (red) dotted line in the above graph. Finally, we apply the associativity of $A,(\mathrm{~A} .12)$, and [Fr, Lem 3.11]. Then we see that the graph in (A.17) exactly matches with the one in (A.11).

Acknowledgements. We would like to thank the organisers of the Oberwolfach Arbeitsgemeinschaft "Algebraic structures in conformal field theories" (April 2007), where this work was started, for an inspiring meeting. We would further like to thank the Hausdorff Institute for Mathematics in Bonn and the organisers of the stimulating meeting "Geometry and Physics" (May 2008). We are indebted to Alexei Davydov, Jens Fjelstad, Jürgen Fuchs, Yi-Zhi Huang, Alexei Kitaev, Urs Schreiber, Christoph Schweigert, Stephan Stolz and Peter Teichner for helpful discussions and/or comments on a draft of this paper. The research of IR was partially supported by the EPSRC First Grant EP/E005047/1, the PPARC rolling grant PP/C507145/1 and the Marie Curie network 'Superstring Theory' (MRTN-CT-2004-512194). 


\section{References}

[AN] Alexeevski, A., Natanzon, S.M.: Noncommutative two-dimensional topological field theories and hurwitz numbers for real algebraic curves. Sel. Math., New Ser. 12, 307-377 (2006)

[Bi] Bichon, J.: Cosovereign hopf algebras. J. Pure Appl. Alg. 157, 121-133 (2001)

[BK] Bakalov, B., Kirillov, A.A.: Lectures on Tensor Categories and Modular Functors. Providence, RI: Amer. Math. Soc., 2001

[C1] Cardy, J.L.: Operator content of two-dimensional conformal invariant theories. Nucl. Phys. B 270, 186-204 (1986)

[C2] Cardy, J.L.: Boundary conditions, fusion rules and the verlinde formula. Nucl. Phys. B 324, 581-596 (1989)

[CL] Cardy, J.L., Lewellen, D.C.: Bulk and boundary operators in conformal field theory. Phys. Lett. B 259, 274-278 (1991)

[DMZ] Dong, C.-Y., Mason, G., Zhu, Y.-C.: Discrete series of the Virasoro algebra and the moonshine module. In: Algebraic Groups and Their Generalizations: Quantum and infinite-dimensional Methods, Proc. Symp. Pure Math. 56, Part 2, Providence, RI: Amer. Math.Soc., 1994, pp. 295-316

[DP] Day, B., Pastro, C.: Note on frobenius monoidal functors. New York J. Math. 14, 733-742 (2008)

[ENO1] Etingof, P.I., Nikshych, D., Ostrik, V.: On fusion categories. Ann. Math. 162, 581-642 (2005)

[ENO2] Etingof, P.I., Nikshych, D., Ostrik, V.: An analogue of radford's $s^{4}$ formula for finite tensor categories. Int. Math. Research Notices 54, 2915-2933 (2004)

[Fe] Felder, G., Fröhlich, J., Fuchs, J., Schweigert, C.: Correlation functions and boundary conditions in rational conformal field theory and three-dimensional topology. Comp. Math. 131, 189-237 (2002)

[FHL] Frenkel, I.B., Huang, Y.-Z., Lepowsky, J.: On axiomatic approaches to vertex operator algebras and modules. Mem. Amer. Math. Soc. 104 (1993)

[Fj] Fjelstad, J., Fuchs, J., Runkel, I., Schweigert, C.: Uniqueness of open/closed rational cft with given algebra of open states. Adv. Theor. Math. Phys. 12, 1283-1375 (2008)

[FK] Fröhlich, J., King, C.: The chern-simons theory and knot polynomials. Commun. Math. Phys. 126, 167-199 (1989)

[Fr] Fröhlich, J., Fuchs, J., Runkel, I., Schweigert, C.: Correspondences of ribbon categories. Adv. Math. 199, 192-329 (2006)

[FRS] Fuchs, J., Runkel, I., Schweigert, C.: Tft construction of reft correlators. I: partition functions. Nucl. Phys. B 646, 353-497 (2002)

[FS] Fuchs, J., Schweigert, C.: Category theory for conformal boundary conditions. Fields Inst. Commun. 39, 25-71 (2003)

[H1] Huang, Y.-Z.: Two-dimensional conformal geometry and vertex operator algebras. Progress in Mathematics, Vol. 148, Boston: Birkhäuser, 1997

[H2] Huang, Y.-Z.: Rigidity and modularity of vertex tensor categories. Comm. Contemp. Math. 10, 871-911 (2008)

[HK1] Huang, Y.-Z., Kong, L.: Open-string vertex algebra, category and operads. Commun. Math. Phys. 250, 433-471 (2004)

[HK2] Huang, Y.-Z., Kong, L.: Full field algebras. Commun. Math. Phys. 272, 345-396 (2007)

[HK3] Huang, Y.-Z., Kong, L.: Modular invariance for conformal full field algebras. http://arxiv.org/abs/ math/0609570v2[math.QA], 2006

[HL] Huang, Y.-Z., Lepowsky, J.: Tensor products of modules for a vertex operator algebra and vertex tensor categories, In: Lie Theory and Geometry, in honor of Bertram Kostant, ed. R. Brylinski, J.-L. Brylinski, V. Guillemin, V. Kac, Boston: Birkhäuser, 1994, pp. 349-383

[JS] Joyal, A., Street, R.: Braided tensor categories. Adv. Math. 102, 20-78 (1993)

[K1] Kong, L.: Full field algebras, operads and tensor categories. Adv. Math. 213, 271-340 (2007)

[K2] Kong, L.: Open-closed field algebras. Commun. Math. Phys. 280, 207-261 (2008)

[K3] Kong, L.: Cardy condition for open-closed field algebras. Commun. Math. Phys. 283, 25-92 (2008)

[Ki] Kitaev, A.: Private communication

[KO] Kirillov, A.A., Ostrik, V.: On $q$-analog of mckay correspondence and ade classification of $\widehat{s l}(2)$ conformal field theories. Adv. Math. 171, 183-227 (2002)

[KR] Kong, L., Runkel, I.: Morita classes of algebras in modular tensor categories. Adv. Math. 219, 1548-1576 (2008)

[Ld] Lauda, A.D.: Frobenius algebras and ambidextrous adjunctions. Theo. Appl. Cat. 16, 84122 (2006)

[Li1] Li, H.-S.: Regular representations of vertex operator algebras. Commun. Contemp. Math. 4, 639-683 (2002)

[Li2] Li, H.-S.: Regular representations and huang-lepowsky tensor functors for vertex operator algebras. J. Alge. 255, 423-462 (2002) 
[Ln] Leinster, T.: Higher operads, higher categories. London Mathematical Society Lecture Note Series 298. Cambridge: Cambridge University Press, 2004

[LP] Lauda, A., Pfeiffer, H.: Open-closed strings: two-dimensional extended tqfts and frobenius algebras. Topology Appl. 155, 623-666 (2008)

[LR] Longo, R., Rehren, K.H.: Local fields in boundary conformal qft. Rev. Math. Phys. 16, 909 (2004)

[Lw] Lewellen, D.C.: Sewing constraints for conformal field theories on surfaces with boundaries. Nucl. Phys. B 372, 654-682 (1992)

[Lz] Lazaroiu, C.I.: On the structure of open-closed topological field theory in two dimensions. Nucl. Phys. B 603, 497-530 (2001)

[Ma] Mac Lane, S.: Categories for the working mathematician. Brelin-Heidelberg-NewYork: Springer, 1998

[Mo] Moore, G.: Some comments on branes, $G$-flux, and $K$-theory. Int. J. Mod. Phys. A16, 936944 (2001)

[MS] Moore, G., Segal, G.: D-branes and K-theory in 2D topological field theory. http://arxiv.org/abs/ hep-th/0609042v1, 2006

[Mü1] Müger, M.: From subfactors to categories and topology II. The Quantum Double of Tensor Categories and Subfactors. J. Pure Appl. Alg. 180, 159-219 (2003)

[Mü2] Müger, M.: Talk at workshop 'Quantum Structures' (Leipzig, 28. June 2007), Preprint in preparation

[O] Ostrik, V.: Module categories, weak hopf algebras and modular invariants. Transform. Groups 8 , 177-206 (2003)

[P] Pfeiffer, H.: Finitely semisimple spherical categories and modular categories are self-dual. Adv. Math. 221, 1608-1652 (2009)

[RT] Reshetikhin, N., Turaev, V.G.: Invariants of 3-manifolds via link polynomials and quantum groups. Inv. Math. 103, 547-597 (1991)

[Se] Segal, G.: The definition of conformal field theory. Preprint 1988; also in: U. Tillmann (ed.), Topology, geometry and quantum field theory, London Math. Soc. Lect. Note Ser. 308, Cambridge: Cambridge Univ. Press, 2004, pp. 421-577

[So] Sonoda, H.: Sewing conformal field theories II. Nucl. Phys. B 311, 417-432 (1988)

[Sz] Szlachányi, K.: Adjointable monoidal functors and quantum groupoids. In: Hopf algebras in noncommutative geometry and physics, Caenepeel, S., Oystaeyen, F.V. (eds.) Lecture Notes in Pure and Applied Mathematics 239 Boca Raton, FL: CRC Press, 2004, pp. 297-307

[T] Turaev, V.G.: Quantum Invariants of Knots and 3-Manifolds. New York: de Gruyter, 1994

[V] Vafa, C.: Conformal theories and punctured surfaces. Phys. Lett. B 199, 195-202 (1987)

[VZ] Van Oystaeyen, F., Zhang, Y.H.: The brauer group of a braided monoidal category. J. Algebra 202, 96-128 (1998)

[W] Witten, E.: Quantum field theory and the jones polynomial. Commun. Math. Phys. 121, 351-399 (1989)

[Y] Yetter, D.N.: Functorial knot theory. Categories of tangles, coherence, categorical deformations, and topological invariants. Series on Knots and Everything 26, River Edge, NJ: World Scientific, 2001

Communicated by Y. Kawahigashi 